\title{
Proceedings of the Intern Section of the Royal Academy of Medicine in Ireland (RAMI) on Saturday 1st February 2020, Venue: The Pillar Centre for Transformative Healthcare, Mater Misericordiae University Hospital, Dublin
}

\author{
(C) Royal Academy of Medicine in Ireland 2020
}

\section{PLATFORM PRESENTATIONS: CASE PRESENTATIONS}

An Analysis Of Hospital Coding Data On Cellulitis Admissions At A Tertiary Referral Centre To Inform Service Development

O'Farrell R, O’Connell J, DeBarra E, McConkey S, McNally C

Infectious Disease Dept., Beaumont Hospital, Dublin

\section{DNE/RCSI}

Background: Cellulitis is a frequent cause of hospital admission to public hospitals1. Our aim was to describe and compare cellulitis hospital admissions under medical and surgical specialties in our tertiary referral centre to inform service development.

Methods: We used the National Quality Assurance and Improvement System (NQAIS) to extract all admissions with cellulitis from 1/1/2018-31/ 12/2018. Data points extracted included age, gender, length of stay, risk factors for cellulitis, readmission rates, Charlson Score and procedures performed. We used the Mann Whitney U test to compare lengths of stay.

Results: There were 250 admissions with cellulitis. 169/250(66.1) were admitted by a surgical specialist, $81 / 250(33.9)$ by a medical. In total there was 2390 bed-days used due to cellulitis. Surgical intervention was required in $2 / 81(2.5 \%)$ admissions under a medical specialist and $8 / 169(4.7 \%)$ of admissions under a surgical specialist. The mean Charlson score $3.53(\mathrm{SD}=6.5)$ in those under a medical specialist and $1.25(\mathrm{SD}=3.4)$ in those under a surgical specialist. Readmission occurred in 19/169(11\%) who were discharged from a surgical specialist and 10/ $81(12.3 \%)$ in those under a medical. The mean LOS was 15.1(SD=30.7) days under medical specialists and $6.9(\mathrm{SD}=10.7)$ days under surgical. There was a statistically significant difference in the mean lengths of stay when those with a Charlson Score of 10 or less was compared with those with a Charlson Score greater than 10 (8.8 days $(\mathrm{SD}=19.8)$ vs. 21 days $(\mathrm{SD}=17.2), \mathrm{p}<0.00001)$. There were 21 different principal specialties managing their care.

Conclusions: There number of cellulitis admissions was high and involved many specialties. The need for surgical intervention was low. Most under surgery required no surgical intervention. An intervention to reduce the number of admissions and length of stay could be the creation of a cellulitis pathway under the direction of a medical specialist and a nurse specialist with clear criteria for surgical admission.

Reference:

1. Activity in Acute Public Hospitals in Ireland 2018 Annual Report, Healthcare Pricing Office
A Review Of Patients On Entresto Attending The Heart Failure Clinic In A Model 3 Hospital In The West Of Ireland

Dervan K, Saeed MU, Lavin F

Cardiology Dept., Mayo University Hospital

\section{WNW/NUIG}

Introduction: Heart Failure is an increasingly common, chronic disease associated with significant morbidity and mortality. Whilst, the prevalence of the disease is approx. $1 \%$ for patients under 65 years old, this increases up to $15 \%$ in those aged over 85 years old.

Today in Ireland, approximately 90,000 people have heart failure at a cost $€ 660$ million a year.

Although heart failure was traditionally regarded as having a poor prognosis, recent advances in management of heart failure has improved prognosis. The Paradigm-HF Study (NEJM, 2014)1 published promising data for "Entresto" (Sacubitril/Valsartan), with regard to improved prognosis in Heart Failure patients with reduced ejection fraction.

Aim: To evaluate the response of patients taking Entresto attending the HF Clinic at Mayo University Hospital

Method: A retrospective review of 31 patients' records attending the Heart Failure clinic in MUH, who have been on Entresto $>3$ months, with initial NYHA Class 2 or more with a reduced ejection fraction $<35 \%$ and on recommended other HF medication as per guidelines.

Results: Thirty-one patient's files were audited for the purpose of this review. Of this cohort 5 are female and 26 are male. Mean age is 67 years old. Only 1 patient had their medication discontinued by their GP. Twelve patients reached the maximum dose of Entresto (97/103mg BD), fourteen were on an intermediate dose $(49 / 51 \mathrm{mg} \mathrm{BD})$ and four remained on the starting dose (24/26mg BD).

The average the ejection fraction, prior to commencing Entresto was $28 \%$ which improved to an average $34 \%$ on treatment. The average BNP was 3,776 , reducing to 1,458 with Entresto. Subjectively, all patients reported an improvement in symptoms.

Conclusion: Allowing for the small cohort, these results show promising results from Entresto with regard to ejection fraction, reduction in proBNP and improvement in quality of life.

\section{Reference:}

1. McMurray J, Packer M, Desai A, Gong J, Lefkowitz M, Rizkala A et al. Angiotensin-Neprilysin Inhibition versus Enalapril in Heart Failure. New England Journal of Medicine. 2014;371(11):993-1004. 
A Presentation Of New Onset Refractory Status Epilepticus (NORSE) Secondary To Newly Diagnosed Neurosyphilis - A Case Report

Murphy R, O’Rourke K

Dept. of Neurology, Dublin Neurological Institute, Mater Misericordiae University Hospital, Dublin

\section{$\mathrm{DML} / \mathrm{UCD}$}

Introduction: We present a case of new onset refractory status epilepticus (NORSE) found to be secondary to neurosyphilis. NORSE is characterised by status epilepticus refractory to at least two antiepileptic medications without a clear aetiology after initial investigations. It is a challenging emergency with significant associated complications and morbidity. Whilst the incidence of seizure in syphilis is between $14 \%$ and $60 \%$, status epilepticus as the presenting manifestation is rare.

Case Presentation: A 31-year-old man from Malawi presented to MMUH ED with a collateral history revealing 5 witnessed tonic-clonic seizures and a background of alcohol excess. The patient was seizing on arrival and seizures proved refractory to levetiracetam, phenytoin, midazolam and ultimately requiring propofol, intubation and ventilation. White cell count was 28.63. EEG demonstrated lateralising abnormalities in the form of left posterior temporal slowing. CT Brain demonstrated no gross abnormality. MRI Brain displayed high signal in the left temporal lobe and deep white matter of the left frontal lobe. Serum autoimmune encephalitis panel was negative. Syphilis serology was positive in serum and CSF. The patient was commenced on $2.4 \mathrm{~g}$ IV Benzylpenicillin and 24 hours later seizure activity had abated. The patient was alert, mobilising and following one step commands. The patient overall did well but did demonstrate persistent gross cognitive deficits on neuropsychological testing on follow-up.

Discussion: NORSE as the first presentation of neurosyphilis is rare but it should be considered in the differential diagnosis for several reasons. Firstly, syphilis is experiencing a resurgence in reported number of cases both in Ireland and overseas (There were 262 more cases of syphilis here in 2019 than 2018 as of 4th December). Secondly, syphilis has a diverse radiological appearance and has been known to mimic both HSV and autoimmune encephalitis. Finally, as demonstrated in this case, timely treatment can mean a relatively prompt improvement in morbidity.

\section{Teamwork Works For A Long Distance Diagnosis}

Corley $\mathrm{L}^{1}$, Murphy $\mathrm{NP}^{1}$, Firth RGR ${ }^{2}$

Dept. of Paediatric Endocrinology ${ }^{1}$, CHI, Temple St, Dublin; Dept. of Endocrinology $y^{2}$, Tallaght University Hospital, Dublin

\section{$\mathrm{DML} / \mathrm{UCD}$}

Background: Neonatal diabetes mellitus (NDM) is rare with reported incidence estimated of 1 in 160,000(1,2). Permanent NDM mostly arises secondary to activating mutations in the K-ATP channel genes KCNJ11 and $\mathrm{ABCC}$. Identification of the underlying genetic mutation may allow switch to oral sulfonylurea therapy which reduces healthcare costs (costbenefits have been calculated to be in the region of 12,000 USD at 10 years following transition from subcutaneous insulin $(3,4)$ ), and improves diabetes control and quality of life.

Case Report: AE, an 8-year old girl from Lomé, Togo, West Africa, diagnosed with NDM at age 2 months was brought to see RF who is working to support diabetes services in Togo. AE's insulin was provided through the IDF Life for A Child Programme. Her regimen involved BD injections of premixed insulin ( 30 units daily). Glycaemic control was suboptimal with significant glycaemic variation.

Establishing her genetic analysis proved exceptionally challenging given the limited resources, postal service, communications and transport networks in her impoverished rural locality. RF liaising with NM in Temple
St, obtained an EDTA sample, carried it by hand to Dublin; it was then sent to the Genomic Laboratory at University of Exeter (who offer free genetic testing for NDM). RF later obtained salivary DNA from AE's parents. This tremendous effort confirmed that $\mathrm{AE}$ had a de novo KCNJ11 missense mutation predicted to be responsive to oral sulphonylurea therapy. She successfully transitioned to oral gilbenclamide with blood glucose levels ranging from 4.0-7.6 mmol/L.

Conclusion/Discussion: This case demonstrates that, in the face of extreme poverty, clinical acumen, persistence, teamwork, and collaboration provided a wonderful outcome for this child and her family. Transition to a simple low-cost treatment allows improved glycaemic control, preventing long-term complications at a substantially reduced cost.

\section{References:}

1. Letourneau, L., Carmody, D., Wroblewski, K., Denson, A., Sanyoura, M., Naylor, R., Philipson, L. and Greeley, S. (2017). Diabetes Presentation in Infancy: High Risk of Diabetic Ketoacidosis. Diabetes Care, 40(10), pp.e147-e148.

2. Grulich-Henn, J., Wagner, V., Thon, A., Schober, E., Marg, W., Kapellen, T., Haberland, H., Raile, K., Ellard, S., Flanagan, S., Hattersley, A. and Holl, R. (2010).

Entities and frequency of neonatal diabetes: data from the diabetes documentation and quality management system (DPV). Diabetic Medicine, 27(6), pp.709-712.

3. Sood, S., Landreth, H., Bustinza, J., Chalmers, L. and Thukaram, R. (2017). Neonatal Diabetes. Journal of Investigative Medicine High Impact Case Reports, 5(1), p.232470961769871.

4. Greeley, S., Letourneau, L. and Philipson, L. (2018). Precision medicine in KCNJ11 permanent neonatal diabetes. The Lancet Diabetes \& Endocrinology, 6(8), pp.594-595.

\section{"A Troublesome Gut": A Case Of Intestinal Failure In A 45-Year- Old Male}

Heneghan $\mathrm{J}^{1}$, Kennedy $\mathrm{N}^{1}$, Chan $\mathrm{C}^{1}$, Costello $\mathrm{M}^{2}$, Joyce $\mathrm{M}^{1}$ Depts. of Colorectal and General Surgery ${ }^{1}$, Nutrition and Dietetics ${ }^{2}$, University College Hospital Galway, Newcastle Road, Galway WNW/NUIG

Introduction: Intestinal failure (IF) is the consequence of a reduction of gut function below the minimum necessary for the absorption of nutrients from the gastrointestinal tract. As a result of this failure, intravenous supplementation is essential. ${ }^{1}$

Case: We describe the case of a 45 -year-old male chef admitted to a university hospital with bloody diarrhoea (30 motions per day), crampy abdominal pain and cold sweats. He was initially identified to have a progressive deterioration of previously diagnosed ulcerative colitis. Following a medical admission, the patient was found to be tachycardic, dehydrated and computed tomography (CT) findings were consistent with acute colitis.

Subsequent CT was suggestive of toxic megacolon. The patient's condition was refractory to medical management. A colorectal surgical opinion resulted in a total colectomy and end ileostomy on day 7 of admission. Histology of the operative specimen gave an impression of pancolitis, most likely ulcerative colitis.

The patient developed an acute septic episode on post-op day (POD) 10. Intravenous fluids (IV) and total parenteral nutrition (TPN) were required as the patient developed a high output stoma ( $>2000 \mathrm{ml} / 24$ hours), resulting in profound dehydration and admission to critical care. MDT input proved imperative while investigations to identify a reversible cause were inconclusive. The patient was eventually weaned off TPN at POD 75 and was discharged home on IV fluids on POD 145.

Discussion: Intestinal Failure has been recently classified as Type 1-3 by European Society for Parenteral and Enteral Nutrition, based on disease onset and anticipated outcomes. 1 Our patient's clinical course and protracted 152-day hospital admission corresponded with a Type 2 Intestinal Failure. This is a relatively uncommon subtype, associated with 
septic and metabolic complications. This type of IF requires TPN over weeks to months, often requires intensive care and management of a multi-disciplinary IF team and carries an in-hospital mortality of 9.6$13 \% .1$

Reference:

1. Pironi L, Arends J, Baxter J, Bozzetti F, Peláez R, Cuerda C et al. ESPEN endorsed recommendations. Definition and classification of intestinal failure in adults. Clinical Nutrition. 2015;34(2):171-180.

\section{Parental Priorities And Their Understanding Of Current Paediatric Outpatient Care}

O’ Callaghan MP, O’ Neill M

Dept. of Paediatrics, Castlebar, Mayo University Hospital

\section{WNW/NUIG}

Background: The paediatric outpatient services serve over 175,000 children in Ireland annually. ${ }^{1}$ Waiting times for these services are ever-increasing, prompting a move from hospitalisation towards ambulatory services in the community. ${ }^{2}$ Little data are available on parental beliefs and expectations from outpatient clinics. It is important to consistently reevaluate our approach to outpatient care and ensure we are meeting healthcare priorities of both patient and parent.

Aim: To clarify parental ideas, concerns and expectations in the paediatric outpatient setting and utilise this information to modernise current paediatric care systems.

Methods: Data were collected via a structured survey that evaluated demographic data, parental priorities at clinic, personal impact and parental understanding of the current outpatient system. A pilot survey with 15 parents allowed for survey refinement in terms of content and comprehension. The study took place over four months in Mayo University Hospital and was extended to general out-patient clinics alone, with no sub-speciality cases. 313 survey results were transcribed into an Excel spreadsheet and analysed with descriptive statistics.

Results: Parental priorities, socio-economic inconvenience of attendance and parental interest in other modes of care delivery were key findings. Highest ranked priorities in clinic attendance included knowledge of the child's diagnosis, an improvement in health and leaving the clinic feeling reassured. Personal impact in attendance at clinic included parking costs, children missing school and excessive waiting times. Parents expressed a keen interest in other care delivery packages, particularly outpatient services lead by a specialist nurse.

Conclusion: Parental expectations and priorities are not currently considered in the paediatric outpatient setting. Understanding parental concerns could play an important role in addressing their visit priorities. Parental interest in alternate service packages may encourage the institution of different forms of future care delivery.

References:

1. A National Model of Care for Paediatric Healthcare Services in Ireland [Internet]. Dublin; 2019 [cited 1 December 2019]. Available from: https:// www.hse.ie/eng/services/publications/clinical-strategy-and-programmes/ health-services-for-children-in-ireland-\%E2\%80\%93-a-guide-to-workforce-planning.pdf

2. A National Model of Care for Paediatric Healthcare Services in Ireland [Internet]. Dublin; 2019 [cited 1 December 2019]. Available from: https:// www.hse.ie/eng/services/publications/clinical-strategy-and-programmes/ model-of-care-for-paediatric-healthcare-integrated-care.pdf

\section{An Acute Dystonic Reaction Secondary To Anti Emetics- A Case Report}

Timon J, Byrnes V

Gastroenterology Dept., Galway University Hospital

\section{WNW/NUIG}

Introduction: An acute dystonic reaction is characterised by involuntary contractions of major muscle groups which leads to unusual movements or posturing. The involvement of the larynx has also been reported and in these cases, the acute dystonic reaction may be life threatening. Acute dystonia's are caused by an imbalance in the basal ganglia where dopamine blockade leads to an excess of cholinergic output. Acute dystonic reactions are most commonly associated with the use of antipsychotics and anti-emetics which work by blocking the D2 receptor such as Metoclopramide and Prochlorperazine.

Case: A 19-year-old male presented to the emergency department with a severe episode of vomiting. This vomiting was difficult to control, and in under 6 hours the patient had been administered; Ondansetron, Metoclopramide, Cyclizine, Prochlorperazine, Diazepam and Haloperidol. Following this, the vomiting ceased. 15 hours later, the patient developed tongue protrusion and lip swelling, the patient remained vitally stable. This was treated as a delayed hypersensitivity reaction with Chlorphenamine and the tongue protrusion immediately improved. This event repeated itself two hours later and again was treated as a hypersensitivity reaction. Following this the patient developed torticollis and held his head turned to the left, it was then realised that the patient was suffering from an acute dystonic reaction. The patient was treated with Promethazine and Midazolam and the dystonia resolved.

Discussion: Acute dystonic reactions are a common and may be a lifethreatening side effect of commonly used medications. The mainstay of treatment is anti-cholinergic medications. The presentation of an acute dystonic reaction can be difficult to recognise. In this case the reaction was partially masked by the use of Chlorphenamine which in addition to its anti-histaminergic effects has a weak anti-cholinergic effect. It is important for all healthcare professionals to be aware of these adverse reactions and to rationalize their prescribing accordingly.

\section{Does Self-Care In Heart Failure Differ Between Patients With Reduced And Preserved Phenotypes?}

Carroll $\mathrm{T}^{1}$, O’Sullivan $\mathrm{C}^{1}$, Egan $\mathrm{D}^{1}$, O’Donoghue $\mathrm{E}^{1,2}$, McDonald $\mathrm{K}^{1,2}$ Cardiology Dept. ${ }^{1}$, St. Vincent's University Hospital, Dublin; Heart Failure Unit ${ }^{2}$, St. Michael's Hospital, Dun Laoghaire, Co. Dublin

\section{$\mathrm{DML} / \mathrm{UCD}$}

Background: Effective self-care is an important aspect of heart failure (HF) management, reducing HF-related hospitalisations and improving quality of life1. The European Heart Failure Self-Care Behaviour Scale (EHFScB 12 point scale) is a reliable, repeatable2 tool used to measure self-care. It is composed of 12 questions (scored 1-5). Four questions relate to patient reported HF signs/symptoms (group A). The remaining eight relate to patient activities that optimise HF management (group B). Chronic heart failure is commonly classified based on left ventricular ejection fraction (LVEF) with patients divided into preserved $(\mathrm{pEF})$ and reduced $(\mathrm{rEF})$ phenotypes. Little is known about the difference in self-care between these groups. Patients with $\mathrm{pEF}$ tend be older 3 with more comorbidities. These factors have been linked to impaired self-care4, possibly impacting self-care adherence in pEF patients.

Aims: To assess the difference in level of self-care between HF-pEF and HF-rEF patients.

Methods: 319 patients from a HF unit with responses recorded to the EHFScB questionnaire were randomly selected. 14 of these were excluded due to incomplete questionnaires.

Patients were divided into $\mathrm{rEF}(\mathrm{LVEF}-<50 \%)$ and $\mathrm{pEF}\left(\mathrm{LVEF}^{3} 50 \%\right)$ subgroups. EHFScBs results were analysed and between-group comparisons were made on total and individual question scores.

Results: 305 questionnaire results were analysed, 163 (53\%) from rEF patients and $142(47 \%)$ from $p E F$ patients. The $\mathrm{pEF}$ group was older with an average age of 75.5 years compared to 67.6 years in the rEF population. The average total score in rEF patients was 21.7 and 21.8 in $\mathrm{pEF}$ 
patients. Group A question scores were 7.2 in $\mathrm{rEF}$ and 7.7 in $\mathrm{pEF}$ patients. Group B scores were 14.6 in $\mathrm{rEF}$ and 14.2 in pEF patients.

Conclusion: This important novel data shows that despite being significantly older, the pEf population had equal self-care adherence compared with $\mathrm{rEF}$ patients being managed in the same unit.

\section{References:}

1- Jonkman NH, Westland H, Groenwold RH, et al. Do Self-Management Interventions Work in Patients With Heart Failure? An Individual Patient Data Meta-Analysis. Circulation. 2016;133(12):1189-98.

2- Shuldham, C. , Theaker, C. , Jaarsma, T. and Cowie, M. R. (2007), Evaluation of the European Heart Failure Self-care Behaviour Scale in a United Kingdom population. Journal of Advanced Nursing, 60: 87-95. doi:10.1111/j.1365-2648.2007.04371.x

3- 2016;133(12):1189-1198. doi:10.1161/CIRCULATIONAHA. 115.018006Lee DS, Gona P, Vasan RS, et al. Relation of disease pathogenesis and risk factors to heart failure with preserved or reduced ejection fraction: insights from the framingham heart study of the national heart, lung, and blood institute. Circulation. 2009;119(24):3070-7.

4- MacInnes, J. (2008). Factors affecting self-care in heart failure: A literature review. British Journal of Cardiac Nursing, 3(7), 293-299. doi:10.12968/bjca.2008.3.7.30495

\section{Gut Wrenching - When Seatbelt Is The Cause}

Smith D, Jennings I, Toor A, Aremu M

Dept. of General Surgery, Connolly Hospital Blanchardstown, Dublin

\section{DNE/RCSI}

Introduction: The widespread use of seatbelts has led to an increase of intestinal injuries following Road Traffic Accidents (RTA's) ${ }^{1}$. Mesenteric Avulsion (MA) is a rare, yet serious complication of blunt abdominal trauma. Caused by sudden deceleration results in tearing /avulsion of the mesentery form it's anatomical attachment. Such forces on the mesentery cause rupture of the visceral blood vessels causing both ischemia to the intestines and haemorrhage. The overall mortality has been recorded as high as $15 \%{ }^{2}$.

Case presentation: We report a case of 51-year old following a high speed road traffic accident (RTA). On presentation he was diaphoretic yet hemodynamically stable. Clinically his abdomen was distended, diffusely tender with a visible seatbelt sign. A FAST scan performed in the emergency department was negative for free fluid. CT abdomen showed a moderate size pelvic hematoma in both the right and left iliac fossae, with blood seen around the liver and spleen. He underwent diagnostic laproscopy which showed ischemic segment of small bowel. Proceeding to exploratory laparotomy, which demonstrated ischemic segment of terminal ileum with avulsion of segment of mesentery. Upon examination of large bowel an avulsion of sigmoid mesocolon was discovered. About $60 \mathrm{~cm}$ of the terminal ileum was resected with side to side small bowel anastomosis and about $15 \mathrm{~cm}$ segment of sigmoid and was brought out as end colostomy.

Discussion: The rarity of mesenteric avulsion, along with its vague presentation can lead to a delay in diagnosis and subsequently intervention ${ }^{2}$. A high index of suspicion combining patient severity of abdominal pain, abdominal examination findings and urgent $\mathrm{CT}$ helps establish diagnosis. A "seatbelt" sign also holds diagnostic value ${ }^{3}$. Given the potential for high morbidity and mortality, it is important to realise patient can have serious injuries even in the absence of clinical and radiological findings. References:

[1] O'Dowd V, Kiernan C, Lowery A, Khan W, and Barry K (2011) Seatbelt Injury Causing Small Bowel Devascularisation: Case Series and Review of the Literature. Emergency Medicine International

[2] Kordzadeh A, Arjun Devanesan A, Parkinson T, Rahim K, and Panayiotopoulos Y (2012) Subtle mesenteric avulsion in a traumatic abdominal wall hernia: A case report Int J Surg Case Rep 2012; 3(9): 417419.
[3] Sharma, Om P.; Oswanski, M.; Kaminski, B.; Issa, N.; Duffy, B; Stringfellow, K; Lauer,S.; Stombaugh, H.(2009) Clinical Implications of the Seat Belt Sign in Blunt Trauma The American Surgeon, Volume 75, Number 9

\section{Hitting The Nail On The Head}

Hughes K, Brophy C, Altawil M, Mohammad M, Riordan F, O'Keeffe N, Sheahan P, Dias A

Dept. of OtoRhinoLaryngology/Head \& Neck Surgery, South Infirmary Victoria University Hospital, Cork

\section{$\mathrm{S} / \mathrm{UCC}$}

A penetrating foreign body injury to the neck can be a life-threatening condition, which poses challenges in terms of primary management, accurate detection and ultimate treatment. The objective of this clinical case report is to highlight the unusual injury of a $3 \mathrm{~cm}$ nail embedded in the neck of a 78-year old gentleman.

Mr BM initially presented to his local hospital after using an electric saw and feeling that a nail darted through his chin into his neck. AP and lateral soft tissue neck x-rays were performed, revealing a metallic fragment embedded in the left neck. In order to ascertain the relationship between the nail and the vascular structures of the neck, a CT neck with contrast was carried out, which showed that the nail was indeed embedded in an anteroposterior direction, in the left parapharyngeal space.

An emergency left neck exploration was performed. The foreign body was found deep to the submandibular gland and deep to the anterior belly of the digastric. Mr BM made a good post-operative recovery and was eventually discharged on oral antibiotics.

While this case had an overall positive outcome, it can be used to illustrate several key learning points. First of all, the size of an entry wound does not correlate with the size of the foreign object, in this case a $3 \mathrm{~mm}$ penetrating wound housed a $3 \mathrm{~cm}$ nail. Secondly, highlighting the importance on flexible nasendoscopy in foreign body injuries, which in this case showed a fullness in the left supraglottic area, corresponding to the position of the nail. Finally, the importance of basic imaging techniques plain film showed the foreign body more clearly than the CT, where there was significant artefact.

The Dynamics Of Anticoagulation In Prothrombotic Individuals With Pulmonary Emboli And Intracranial Haemorrhage

\author{
Morgan $\mathrm{J}^{1}$, Basit $\mathrm{M}^{2}$ \\ Medical Dept. ${ }^{1}$, Medicine for the Elderly/General Medicine ${ }^{2}$, Our Lady \\ of Lourdes Hospital, Drogheda
}

\section{DNE/RCSI}

A 63-year-old male presented to the emergency department complaining of a week's history of chest tightness, a dry cough and intermittent shortness of breath. The Wells score was found to be 1.5 and the differential included a pulmonary embolus. A positive D-dimer and a subsequent CTPA confirmed multiple pulmonary emboli and a possible left ventricular thrombus. Echocardiogram showed an apical LV thrombus. In any case anticoagulation is a balance of risks between clotting and haemorrhage but this is particularly pertinent due to the history.

A previous cryptogenic stroke in 2017 and the LV thrombus increases the risk of a repeat stroke. His medical history is relevant for recurrent DVTs and the insertion of an IVC filter. Most importantly; in September 2019; he suffered a large subdural haematoma and had a burr hole procedure performed. A repeat CT brain on November 1st showed enlargement of the haematoma. A follow up CT brain on November 11th showed an acute on chronic bleed but the patient was asymptomatic and discharged with advice to return if he deteriorates. 
Currently the patient has multiple pulmonary emboli, a LV thrombus and a chronic resolving subdural haematoma. The dynamics between these three conditions is precarious and the need to anticoagulated is offset by the potential for the subdural haematoma to acutely bleed. After much discussion with neurosurgery; haematology; other geriatricians; cardiology and the patient himself; it was decided to anticoagulate with unfractionated heparin as it is believed that the benefit outweighs the risk; there is a reversal agent and it requires regular APTT ratio monitoring. The patient is currently progressing well on his infusion.

This case is the perfect example of how practicing medicine is never black and white; how decisions are fraught with risk and how multidisciplinary healthcare; expertise and teamwork is an absolute necessity.

"Mackerel's Diverticulitis": The Value Of Laparoscopy In A Case Of Perforation Of A Meckel's Diverticulum By A Swallowed Fish Bone - A Case Report And Discussion

Harris L ${ }^{1}$, O’Keeffe G ${ }^{1}$, Brady A ${ }^{2}$, O’Sullivan A ${ }^{1}$

Depts. of Surgery ${ }^{I}$, Radiology ${ }^{2}$, Mercy University Hospital, Cork

$\mathrm{S} / \mathrm{UCC}$

Introduction: Meckel's diverticulum is the most common congenital abnormality of the gastrointestinal tract. It is present in between $2 \%$ and $4 \%$ of the population. It arises due to persistence of the congenital vitellointestinal duct. Perforation by a foreign body is an extremely rare complication of a Meckel's diverticulum.

Case Presentation: A 58-year-old man presented to hospital with a threeday history of sudden onset pain in the right iliac fossa, increasing in severity with associated radiation to the pelvic region. He was otherwise asymptomatic. On examination he was tender in the RIF, with rebound and guarding present. Despite analgesia and antibiotic therapy his pain persisted and so a diagnostic laparoscopy was scheduled. An in-tact fish bone was visualized, perforating a Meckel's diverticulum through and through. A laparoscopic stapled diverticulectomy and an appendectomy were performed, and the patient recovered well. He was discharged fortyeight hours later with five days of oral antibiotics and appropriate analgesia.

Discussion: Complications occur most commonly in childhood, often presenting as gastrointestinal bleeding. The most common complications of a Meckel's diverticulum in adults include inflammation and intestinal obstruction. Perforation by a foreign body accounts for approximately $8 \%$ of cases of complicated Meckel's diverticula. A consensus exists that symptomatic Meckel's should be managed surgically. However, radiological investigations often fail to establish the diagnosis preoperatively and clinically it frequently mimics other conditions such as acute appendicitis. Laparoscopy appears to be a safe diagnostic and therapeutic tool in such cases. It has been shown to significantly reduce delays in diagnosis and management, which is imperative in those who present with peritonitis. Conclusion: Perforation by an ingested fish bone is a very rare complication of a Meckel's diverticulum. Laparoscopy is key in both diagnosing and treating such patients in a safe, timely, and cost-effective manner.

\section{Adversities In End-Of-Life Care For Nephrology Patients: A Case Report}

Ni Sheaghdha A, De Freitas D

Nephrology Dept., Beaumont Hospital, Dublin

\section{DNE/RCSI}

Introduction: Through the use of a case study, the author demonstrated the many challenges to providing adequate end-of-life care to renal dialysis patients.

Case Presentation: A 90-year old gentleman presented with aspiration pneumonia on a background history of end-stage kidney disease, on haemodialysis thrice weekly for the past six years. During the course of his admission he generally deteriorated was made palliative at a multidisciplinary meeting.

The patient faced many adversities in regards to his palliative care including: hospital single room shortages to provide privacy and dignity to the patient and his grieving family, waiting lists for a hospice bed, inappropriate use of haemodialysis in a dying patient, sub-optimal training and knowledge in end-of-life care amongst junior doctors, the ethical dilemma regarding use of naso-gastric feeding in a dying patient, the unpredictable nature of end-stage renal disease.

Conclusions: Renal dialysis patients are $50 \%$ less likely to receive hospice services in comparison to other diagnosis for a wide array of reasons including the unpredictable course of end-stage renal disease. Dialysis should only be considered in renal patients if it will improve renal function or has the potential to extend the patient's life. Patient's often regretted their decision to continue dialysis whilst dying due to being influenced by family and medical staff. Naso-gastric feeding in a dying patient is medically futile and increases the risk of complications.

The 'Design and Dignity Programme' in Ireland provides grants to hospitals to provide spaces to enable respect and privacy in dying patients. However, there is a major shortage of single patient rooms to accommodate for a $42 \%$ increase in palliative care patients by 2020 . There is a need for mandatory palliative care education for intern doctors as gaps in knowledge and communication skills regarding end-of-life management can lead to sub-optimal patient deaths and harbour psychological distress amongst NCHDs.

\section{References:}

1. Amy Rak, Rupesh Raina, Theodore T. Suh, Vinod Krishnappa, Jessica Darusz, Charles W. Sidoti, Mona Gupta, Palliative care for patients with end-stage renal disease: approach to treatment that aims to improve quality of life and relieve suffering for patients (and families) with chronic illnesses, Clinical Kidney Journal, Volume 10, Issue 1, 1 February 2017, Pages 68-73, https://doi.org/10.1093/ckj/sfw105

2. Krishna, L. (2011). Nasogastric feeding at the end of life: A virtue ethics approach. Nursing Ethics, 18(4), 485-494.https://doi.org/10.1177/ 0969733011403557

3. Linane, H., Connolly, F., McVicker, L. et al. Disturbing and distressing: a mixed methods study on the psychological impact of end of life care on junior doctors. Irish Journal of Medical Science (2019) 188: 633. https:// doi.org/10.1007/s11845-018-1885-z

4. Barbara A Head, Tara J Schapmire, Lori Earnshaw, John Chenault, Mark Pfeifer, Susan Sawning, Monica A Shaw: Imroving medical graduates' training in palliative care: advancing education and practice. Advanced Medical Education Practice Journal. . 2016; 7: 99-113 .Published online 2016 Feb 24. doi: 10.2147/AMEP.S94550

5. University College Cork (UCC), School of Nursing and Midwifery, All Ireland Institute of Hospice and Palliative Care (AIIHPC); Evaluation of the Design and Dignity Programme. The Irish Hospice Foundation. Website: https://hospicefoundation.ie/wp- content/uploads/2019/03/ DD_Evaluation_Report_Final_2019.pdf

6. Pauline M Kane, Barbara A Daveson, Karen Ryan, Regina McQuillan, Irene J Higgins, Fliss E M Murtagh. The Need for Palliative Care in Ireland: A Population-Based Estimate of Palliative Care Using Routine Mortality Data, Inclusive of Nonmalignant Condition. Journal of Pain and Symptom Management. Vol 49, Issue 4. April 2015. Pages 726-733.

\section{Collagenopathy At The Neurology Clinic: A Case Series}

Dunne AK, Allen NM

Dept. of Paediatrics, National University of Ireland Galway, \& Galway University Hospital, Ireland

\section{WNW/NUIG}

Aims: The collagenopathies are a diverse group of heritable connective tissue disorders caused by defects of collagen biosynthesis ${ }^{1}$. They may 
present elusively if the clinician is not on the "look out", and patients may experience severe complications if left undiagnosed. We describe three, of a series of collagenopathies, presenting to the neurology clinic for suspected neurological disorder.

Results: Case 1: An 18-month-old male with hypotonia and gross motor delay referred for investigation of underlying neuromuscular disorder. $\mathrm{He}$ had a herniotomy/orchidopexy at 16 months, with sutures noted to be "difficult to insert". Examination revealed macrocephaly with frontal bossing, low set ears, translucent, soft, doughy skin with easy bruising, acrogeric peripheries, vertical talus and pectus excavatum. There was significant joint hypermobility but no muscle weakness. Ehlers Danlos syndrome was suspected. Exome sequencing revealed a de novo dominant mutation in COL3Al verifying grave diagnosis of vascular Ehlers-Danlos. ${ }^{2}$

Case 2: A five-year-old male was referred for investigation of staring episodes which were non-epileptic. He was noted however, to have a background of mild sensorineural hearing loss and high myopia. Family history was significant for retinal detachment (Mum). Examination revealed joint hypermobility. Genetic testing of both revealed a splice mutation in COL2A1 confirming a diagnosis of Stickler syndrome. ${ }^{3}$

Case 3: Female full-term infant born to consanguineous Traveller parents noted at five months to have right-sided hemiplegia. MRI brain revealed leftsided porencephaly. Male sibling, born full-term had antenatal ventricular dilatation, MRI revealed right-sided porencephaly. Due to familial porencephaly, molecular analysis of COLAA1 was investigated (negative), but $C O L 4 A 2$ analysis revealed a missense mutation, predicted pathogenic.

Conclusion: Collagenopathies affect multiple organ systems with phenotypic variability and can therefore present to a wide variety of specialities, leading to significant diagnostic challenges. The importance of early recognition enables parental and genetic counselling, and significantly aids supportive treatment and anticipatory care.

\section{References:}

Jobling R, D'Souza R, Baker N, Lara-Corrales I, Mendoza-Londono R, Dupuis L, Savarirayan R, Ala-Kokko L, Kannu P. The collagenopathies: review of clinical phenotypes and molecular correlations. Current rheumatology reports. 2014 Jan 1;16(1):394.

Malfait F, Francomano C, Byers P, et al. 2017. The 2017 international classification of the Ehlers-Danlos syndromes. Am J Med Genet Part C Semin Med Genet 175C:8-26.

Robin NH, Moran RT, Warman M, Ala-Kokko L. Stickler syndrome.In: Pagon RA, Adam MP, Bird TD, et al. editors. GeneReviews ${ }^{\mathrm{TM}}$ [Internet]. Seattle (WA): University of Washington, Seattle; 1993-2013. 2000 June 9 [updated 2017 Mar 16]. http://www.ncbi.nlm.nih.gov/books/NBK1302/

\section{A Descriptive Study Of Fractures Of The Cervical Spine Managed By The Neurosurgery Department At Cork University Hospital}

Mulcahy C, O'Sullivan MGJ, Lim MS
Dept of Neurosurgery, Cork University Hospital

$\mathrm{S} / \mathrm{UCC}$

Background: Fractures of the cervical spine are common ${ }^{1}$ and can lead to significant mortality and morbidity ${ }^{2}$. Cork University Hospital (CUH) is one of two neurosurgical centres in the Republic of Ireland managing cervical spine fractures; it serves a population of 1.3 million $^{3}$.

Objectives: To describe the demographics, management and outcomes of patients with cervical spine fractures managed by the CUH neurosurgery department, and to assess for trends in this data.

Design: A retrospective chart review.

Setting: Cork University Hospital, a Level 1 Trauma center.

Participants: In the two-year period 2016 and 2017, 66 patients with confirmed cervical spine fractures were identified.

Results: Forty-one (62.1\%) patients were male. Median age was 57 years.
There was a significant majority of males aged 57 and under (26 males, 8 females), and slight majority of females over 57 years old (15 males, 17 females).

C5 was the most commonly fractured vertebra (30.3\%), followed by $\mathrm{C} 2$ and C6 (25.8\%).

The most common causes were: Fall from a height above ground level (30.3\%), Road Traffic Accidents (27.3\%), Ground-level fall (13.6\%), Fall from horse $(10.6 \%)$.

A trend between cervical spine fractures as a result of falling from a height and alcohol consumption prior to the fall was observed.

Rigid collars were used to manage $83.3 \%$ of patients; Halo immobilisation was used in $4.6 \%$, and surgical intervention was performed in $12.1 \%$. At the most recent follow-up, $62.1 \%$ of patients were asymptomatic. Furthermore, radiologically, the fracture(s) had fully healed in $22.7 \%$ of patients and were stable but not yet fully healed in $40.9 \%$.

Conclusions: The study demonstrated that patients aged 57 and under were much more likely to be male, those over 57 were almost equally likely to be male or female.

Falling from a height was the most common cause of cervical spine fractures, followed by road traffic accidents. Alcohol was implicated in many of the cervical spine fractures, particularly in those that occurred as a result of falling from a height. The vast majority of patients were managed with a rigid cervical collar.

\section{Non-Invasive Prenatal Testing In Pregnancy In An Irish Cohort}

Traynor B, McCarthy F

Dept. of Obstetrics, Cork University Maternity Hospital

\section{S/UCC}

Objectives: To assess the demographics, clinical indications and test results of a cohort of pregnant patients undergoing non-invasive prenatal testing (NIPT) in 3 Cork obstetric centres. To examine the cost implications of provision of NIPT in the 3 centres.

Design: Retrospective cross-sectional study

Setting: Clinical records from Cork University Maternity Hospital (CUMH), Cork Women's Clinic (CWC) and Cork University Hospital Consultant's Private Clinic (CPC).

Participants: All women who underwent NIPT in the 3 Cork obstetric centres between August 2015 and September 2018.

Outcome Measures: Demographics, clinical indications and test results. Results: 1,258 patients were involved in this study. $92.9 \%$ were low risk. $0.4 \%$ were high risk for $\mathrm{T} 13,1.3 \%$ were high risk for $\mathrm{T} 18$ and $2.6 \%$ were high risk for T21. A redraw was required for $2.7 \%$. The mean gestational age at testing was 14 weeks. Mean age of patients was 36.5 years with $71.9 \%$ being $\geq 35$ years old. No statistically significant difference in NIPT results for patients $\geq 35$ vs. $<35$ was found $(\mathrm{p}=0.136$ ). Raised nuchal translucency was the most common clinical indication $(n=31) .3 .4 \%$ of patients with no indication were high risk, compared to $29 \%$ of patients with raised nuchal translucency ( $p<0.001), 60 \%$ of patients with a cystic hygroma $(\mathrm{p}<0.001)$ and $22.2 \%$ of patients with IUGR $(p=0.037) .5 .7 \%$ of patients from the public centre were high risk compared to $3.2 \%$ from the private centres $(\mathrm{p}=0.037)$. A cost of $€ 5,120$ in CUMH, $€ 8,288$ in $\mathrm{CPC}$ and $€ 14,192$ in CWC was incurred per 1 positive result found

Conclusion: Clinical indications of raised nuchal translucency, cystic hygroma and IUGR increased the likelihood of a high-risk result. Patients from CUMH had a greater proportion of high-risk results compared to the two private centres. The cost of NIPT per positive result is significant and requires further cost benefit analysis.

\section{PLATFORM PRESENTATIONS: CLINICAL RESEARCH}


When Obsessions Become Delusions: A Clinical Dilemma. Case Report

Murray N, Bambury A

North Lee Mental Health Service

\section{$\mathrm{S} / \mathrm{UCC}$}

Background: The relationship between obsessions and delusions has important nosologic and clinical implications in psychiatry. They are key features of a number of psychiatric disorders and correct identification determines accurate diagnosis. Recent investigations suggest that the boundary between obsessions and delusions may not be dichotomous, but that they exist on a continuum of insight. We report the case of a 23 -yearold Irish male with a long-standing diagnosis of obsessional compulsive disorder (OCD) who developed obsessional thoughts regarding his sexual orientation. His obsessions evolved into what is thought to be delusions regarding paedophilia.

Objectives: To explore the relationship between obsessions and delusions in major psychiatric disorders; both in relation to this case and recent research.

Methods: Case report and key paper review.

Results: The patient developed obsessional thoughts regarding homosexuality and, over time, paedophilia. He reported intrusive, distressing thoughts regarding children and a compulsion to tell others that he was a paedophile. A full psycho-sexual assessment was conducted. No evidence of was paraphilia found. He maintained a fixed false belief that he was a paedophile and became distressed to the point of suicidality. The patient's treatment was changed from atypical anti-psychotic Quetiapine to tricyclic anti-depressant Clomipramine. In due course, intrusive thoughts lessened and mood lifted. His delusion of being a paedophile subsided and so too his compulsion to tell others.

Conclusion: This case demonstrates that, in terms of phenomenology, it can be difficult to differentiate between obsessions and delusions. Research has shown that the combination of antidepressant and anti-psychotic can be of benefit in the treatment of depression with psychosis. Similarly, the addition of atypical antipsychotic can improve symptoms of OCD. Here, a marked improvement was seen with the commencement of a tricyclic antidepressant. It is felt that this is the case of a patient with OCD and loss of insight.

\section{An Investigation Into Adolescent Sports Related Injuries Presenting To An Irish Emergency Department}

Maher L, Perry I

Dept. of Epidemiology and Public Health, University College Cork

$\mathrm{S} / \mathrm{UCC}$

Objectives: The primary objective of this study was to establish the incidence of 13-19 year olds presenting to an Irish Emergency Department (ED) with Sports Related Injuries (SRIs) and to compare rates across individual sports. A secondary objective was to provide an up-to-date description of the demographics of SRIs presenting to an Emergency Department.

Design: Descriptive epidemiological study.

Setting: Tallaght Hospital (an Irish University Hospital) Emergency Department.

Participants: All 13-19 year olds presenting to the ED with a true SRI between 1st January and 31st December 2014. For a case to be included, it must have been the first time presentation of an acute injury that happened within the normal practice of sport.

Results: Over the 12-month period, 8401 13-19 year olds presented to Tallaght Hospital ED with injuries. Of these, 1735 cases $(20.7 \%$ of injury attendances) were due to SRI. The overall SRI incidence was 41.7 (39.7-43.7) injuries/1000 persons/year. Football was the most frequently implicated sport, with 15.5 (14.4-16.8) injuries/1000 persons/year. Rugby carried the second highest injury rate, with 5.7(5.0-6.4) injuries/1000 persons/year. $24.6 \%$ of rugby-related presentations were due to head injury, thereby giving rugby a 2.3 times $(\mathrm{p}<0.0001)$ relative risk of head injury. Head injury was associated with a 5.2 times $(p<0.0001)$ relative risk of admission to hospital.

Conclusion: The results of this study provide a useful insight into the incidence of adolescents presenting to an Irish Emergency Department with Sports Related Injuries, as well as providing an up-to-date description of these injuries. Adolescents, particularly males in their early teenage years, are at a significant risk of Emergency Department presentation with a SRI in any given year. It is clear that rugby carries a worryingly high risk of head injury. Exact sports participation numbers are needed to quantify the risk of a rugby player sustaining a head injury in a given year.

Alcohol Involvement In Hospital-Treated Self-Harm Of Patients Under 25 Years Of Age In Ireland From 2013-2016: A Descriptive Study

Hilmi $\mathrm{H}^{1}$, Griffin $\mathrm{E}^{2}$, Perry $\mathrm{IJ}^{3}$

School of Medicine ${ }^{1}$, University College Cork; National Suicide Research Foundation ${ }^{2}$, Cork; School of Public Health ${ }^{3}$, University College Cork, Cork

\section{S/UCC}

Background: Self-harm is common in adolescence with increasing rates in teenage years. Alcohol involvement is among factors known to be often involved in hospital-treated self-harm. It is essential to establish the role of alcohol in self-harm as well as exploring the different characteristic between presentations with and without alcohol.

Methods: Data on self-harm presentations to acute hospitals Emergency Departments in Ireland from 1 January 2013 to 31 December 2016 were analysed. The characteristic of self-harm patients presenting with and without alcohol consumption were compared. The factors associated with alcohol-involved self-harm were identified using univariate and multivariate logistic regression.

Results: A total of 15415 self-harm presentations between the age of 10 to 24 years old were recorded. Alcohol was present in $22.4 \%$ of the presentations. The factors associated with alcohol involvement is male, age 20-24 years old, presenting during out of hours, having engaged in drug overdose and attempted drowning. Patients with alcohol on board were more likely to be brought in by ambulance but were less likely to be admitted.

Conclusions: This study highlights the prevalence of alcohol involvement in self-harm presentations (1 in 5) among young people as well as, identifies the factors associated with presentations involving alcohol. Appropriate out-of-hours services were required to manage these presentations including ambulances, psychiatric assessments and paediatric services. It also raises legislation issue for tighter alcohol control.

Analysis Of Patient Population Presenting With Proliferative Diabetic Retinopathy Identified And Referred From Irish National Screening Program, Retinascreen

Powell $\mathrm{S}^{2}$, Landi $\mathrm{L}^{1,2}$, Blaaow $\mathrm{K}^{1,2}$, Abdelrahman $\mathrm{M}^{2}$, Pandey $\mathrm{R}^{1,3}$, Keegan $\mathrm{D}^{1,2}$

Diabetic RetinaScreen ${ }^{1}$, National Screening Service, HSE; Dept. of Ophthalmology $y^{2}$, Mater Retinal Research Group ${ }^{3}$, Mater Misericordiae University Hospital, Dublin

\section{$\mathrm{DML} / \mathrm{UCD}$}

Due to the risk of severe vision loss associated with proliferative diabetic retinopathy (PDR), the RetinaScreen program has pathways designed for urgent PDR referrals. In this retrospective analysis, we report on the population and treatment needs of previously PRP-treated (R3P1) and PRP-naive (R3P0) (PDR) patients screened and referred for treatment to the Mater Hospital Diabetic Retina Treatment (DRT).

From January 2016 to December 2017, 196,003 patients were screened under the National Diabetic Retinal Screening Program. Medical records 
of all patients referred with PDR were analyzed. Data was collected regarding retinopathy grade, photocoagulation procedures, anti-VEGF injections, OCT central subfield thickness (CST), best-measured visual acuity, and history of previous ocular treatments. Snellen visual acuity was converted to LogMAR visual acuity scale for statistical analysis.

4,515 referrals were made to the Mater DRT from RetinaScreen. 2,935 patients were referred with diabetic retinopathy. 242 patients (409 eyes) had PDR (173 binocular, 63 monocular). 245 eyes (59.9\%) were graded R3P1 and 164 eyes (40.1\%) were graded R3P0. From the 245 eyes R3P1 cohort 100 eyes (R3sM0P1) were graded with stable PDR, and of the remaining $145 \mathrm{R} 3 \mathrm{aP} 1$ eyes: 110 eyes $(75.9 \%)$ were given additional PRP (R3aM0P1), 3 eyes (2\%) were treated with anti-VEGF injections and PRP (R3aM1P1), and 32 eyes $(22.1 \%)$ were treated with anti-VEGF alone (R3sM1P1). From the 164 PRP-naive eyes (R3P0) 146 eyes (89\%) (R3aM0P0) were treated with PRP; 14 eyes $(8.5 \%)$ (R3aM1P0) were treated with PRP and anti-VEGF injections and 4 eyes (2.4\%) (R3aM1P0) were managed with anti-VEGF alone. 14 eyes $(8.5 \%)$ of the R3aP0 group were managed with a combination of PRP, focal laser, and anti-VEGF injections. RetinaScreen has been very successful at identifying proliferative disease. A significant number of treated eyes required further laser indicating the prevalence of undertreated PDR in Ireland prior to RetinaScreen.

\section{Peripartum Angiopathy - A Rare Cause Of Intrapartum- Intracranial Haemorrhage}

Boylan M, Larkin C

Dept. of Anaesthetics \& ICU, Beaumont Hospital, Dublin

\section{DNE/RCSI}

Introduction: Haemorrhagic strokes are rare in pregnancy however they are responsible for significant maternal morbidity and mortality. Reversible cerebral vasoconstriction syndrome (RCVS) is a clinical-angiographic syndrome characterized by sudden onset severe headaches, seizures, focal neurological deficits and segmental dilatation and narrowing of large and medium sized cerebral arteries. Vasoconstriction caused in pregnancy or puerperium is known as post-partum angiopathy, a recognized subset of RCVS. We present a case of spontaneous intracranial haemorrhage following induction of labour. Case Report: A 36-year-old primigravida patient was induced with syntocinon at 38 weeks gestation for intrauterine growth restriction (IUGR). Her pregnancy had been uneventful with no evidence of hypertension, proteinuria or preeclampsia. She complained of a sudden onset severe headache and became drowsy. At this point, there was a prolonged foetal bradycardia and he patient underwent an emergency, GA caesarean section with delivery of a healthy baby. Post-operatively, she did not regain consciousness and had generalized tonic-clonic seizures, which were treated with midazolam, phenytoin and levetiracetam.

CT brain demonstrated a right basal ganglia intraparenchymal haematoma with intraventricular extension and mild ventriculomegaly. CT angiogram showed no obvious aneurysm or arteriovenous malformation, but there was widespread constriction of her arterial intracranial vessels.

The patient was transferred to our institution, received a bolus and infusion of magnesium sulphate and underwent a decompressive craniectomy with evacuation of intraparenchymal haematoma. Subsequent angiography confirmed that there was no underlying vascular malformation to account for the ICH. She remained in ICU for 10 days and was transferred back to the referring hospital for ongoing stroke rehabilitation with a left-sided hemiparesis.

Conclusion: Peripartum angiopathy is a rare ill-defined phenomenon associated with strokes, both ischaemic and haemorrhagic in the peripartum period. CT imaging identifies abnormalities in the intracranial vessels in the absence of underlying malformation. The potential overlap with eclampsia is unclear, as is the optimum treatment approach.
The Surgical And Post-Operative Management Of Peritoneal Carcinomatosis In The Younger Patient - A Case Report

McInerney N, Kelly M, Mulsow J

Dept. of Colorectal Surgery, Mater Misericordiae University Hospital, Dublin

\section{$\mathrm{DML} / \mathrm{UCD}$}

Background: Cytoreductive surgery and Heated Intra-peritoneal Chemotherapy (CRC/HIPEC) is the standard of care for peritoneal metastases from abdominal and pelvic malignancies. HIPEC/CRS involves resecting the peritoneum and involved viscera, and then instilling heated chemotherapy into the peritoneal cavity.

Case: We report the case of a 19-year-old male referred to the National Peritoneal Malignancy Centre with peritoneal involvement from a colonic tumour at the hepatic flexure. He initially underwent neoadjuvant chemotherapy, but required emergency divertingileostomy due to obstruction during treatment. Diagnostic staging laparoscopy confirmed a peritoneal carcinomatosis index (PCI) score of 23. Extracolonic peritoneal involvement was identified in the pelvis, small-bowel, spleen and the ileostomy site.

He was counselled extensively regarding his poor tumour biology, but was keen to proceed with CRS/HIPEC. At surgery, he underwent subtotal colectomy, peritonectomy, omentectomy, small-bowel resection including anterior wedge of D2 with a shave margin from head of pancreas. Mitomycin-C HIPEC was administered for one hour at 41C, and a new end-ileostomy was formed.

Post-operatively his course was complicated with a contained duodenal collection (leak), that was managed conservatively with TPN, and drainage. He was discharged home day 25 . He subsequently regained $30 \%$ of previously lost weight, is tolerating light diet and will soon commence adjuvant chemotherapy.

Discussion: Recently, there have been reports highlighting the increased incidence of advanced colorectal malignancies in younger patients. This has substantial challenges regarding patient counselling and quality of life implications. The use of decision aids helps educate patients regarding the risk and complexity of multivisceral resections and provides real-world expectations in the post-operative phase.

Conclusion: The management of peritoneal carcinomatosis has evolved significantly. Currently, more aggressive surgical resections are performed in 'select patients' offering the chance of long-term survival. Increasingly, younger patients are presenting with advanced cancers that present a difficult challenge regarding pre-operative counselling and managing expectations.

Vascular Involvement Following Medial Migration Of Acetabular Components Post-Total Hip Replacement: A Case Series

Chee SY, Lee JJ, Syed AZ, Tubassam M

Dept. of Vascular Surgery, Galway University Hospital

\section{WNW/NUIG}

Background: Vascular involvements following primary or revision of total hip replacement (THR) is rare $(<1 \%)$. However, it often leads to significant morbidity and mortality. The risk of total mortality is $7 \%$, $15 \%$ risk of radical lower extremity amputations and $4 \%$ risk of more selective amputations.

Methods: We present a retrospective case series of three patients who underwent revision THR due to medial migration of acetabular component in 2019. All of our three patients were elderly with multiple comorbidities. The mean delay between last surgery and the current surgery due to vascular complications was 46.5 months (range 0.5 to 120 months). 
Results: No complications were noted on post-operative follow-ups in clinic with X-ray. The patients showed significant clinical improvements. Conclusions: These cases highlight the interesting approaches used during re-revision of total hip replacements which involve the vasculature.

\section{An Audit of Caesarean Sections Among Primigravid Women in University Maternity Hospital Limerick}

Dunlop K, Popescu C, Imcha N, Mantas A

Dept. of Obstetrics \& Gynaecology, University Maternity Hospital

Limerick, Limerick

\section{$\mathrm{MW} / \mathrm{UL}$}

Introduction: The number of caesarean sections (CS) performed is increasing steadily worldwide. Within our own hospital, we noted a significant number of primigravid women undergoing CS. CS increases morbidity in future pregnancies. We therefore audited the indications for CS in primigravid women, in order to identify areas for future clinical improvement.

Methods: This audit was a retrospective chart review of women who delivered at UMHL in the month of January 2019. All primigravid women were identified, and those who were delivered by CS underwent chart review. A number of variables, including maternal age, gestation, maternal risk factors, and indication for CS were recorded.

Results: 43 charts were reviewed. The mean age was 31 years $+/-1$ year, with a range of $17-50$. Mean gestation was $38+5+/-2$ days. $20.9 \%$ of cases were spontaneous onset of labour, $41.9 \%$ were inductions of labour, and $37.2 \%$ of women were never in labour. Maternal risk factors influencing the decision for CS included pre-existing maternal disease, gynaecological reasons (history of cervical treatments, recurrent miscarriages), advanced maternal age, high BMI, and pregnancy-related reasons (large for dates, meconium, group B streptococcus, pre-eclampsia, multiple gestation). The most common indications for CS were abnormal fetal position (37\%), failure to progress $(30 \%)$, abnormal CTG $(26 \%)$, pre-existing maternal disease or gynaecological procedure (19\%), and large for dates baby $(16 \%)$.

Discussion: This audit highlights a large number of primigravid mothers undergoing CS. This has implications and risks to subsequent pregnancies, including morbidly adherent placentae, which could be diminished. In the future, we wish to extend the audit to a six-month period in order to gather more significant data and identify areas for future clinical improvement which may reduce the number of primigravid women undergoing CS

Dry Cough, Exertional Desaturation, Hypercalcaemia - An Unusual Late Presentation Of PCP Post-Transplant

O'Farrell-Tyler A, Malik F, Holian J, O'Riordan A

Dept. of Nephrology, St. Vincent's University Hospital, Dublin

\section{DML/UCD}

Introduction: Pneumocystis pneumonia (PCP) is an infection that typically presents with dyspnoea, dry cough and pyrexia. At risk patients include HIV-infected patients, those with pre-existing lung disease and patients undergoing immunosuppression including solid organ transplant recipients. We present the case of a 65 -year old female patient with an atypical manifestation of PCP, 17 years post-transplant.

Case: She had a liver transplant in 1993 for primary biliary cirrhosis (PBC). She developed chronic kidney disease due to focal segmental glomerulosclerosis and recurrence of $\mathrm{PBC}$, which required combined liver and kidney transplant in 2002. Pre-admission, she was taking regular immunosuppression, tacrolimus and mycophenylate, and generally well with no recent illnesses. Presentation was with chest pain, dry cough, exercised-induced oxygen desaturation, with hyponatraemia (127mmol/ 1) and hypercalcaemia $(3.14 \mathrm{mmol} / 1)$. Initial chest radiographs were unremarkable. The hypercalcaemia proved resistant to usual treatment and the dyspnoea worsened. Bronchoscopy and CT thorax detected pneumocystis jirovecii and ground glass opacification. The patient was started on trimethoprim-sulfamethoxazole, but later switched to atovaquone due to hyperkalaemia. Intensive care input was required for ventilation and dialysis for acute kidney injury. Subsequently, the patient completed 21 days of antimicrobials, and was discharged when renal function and calcium returned to baseline.

Discussion: This case highlights some atypical features; the timing of PCP infection post-transplant and the presence of hypercalcaemia. She was not on PCP prophylaxis, as the highest risk of opportunistic infection is 6-12 months post-transplant. However, PCP can occur years posttransplant e.g. complication of increased immunosuppression treatment. Hypercalcaemia is an unusual manifestation with PCP but is likely due to calcitriol production by activated pulmonary alveolar macrophages, independent of PTH. There is an increase in PCP worldwide, likely due to more widespread use of potent immunosuppressants, so this case is a reminder to consider opportunistic infections in immunocompromised patients who present generally unwell.

\section{Double Diabetes: The Importance Of Always Considering The Differential}

Hoban J, Mc Donnell C

Dept. of Paediatric Endocrinology, CHI, Temple St. Dublin

\section{$\mathrm{DML} / \mathrm{UCD}$}

Aim: The purpose of this case report was to highlight to clinicians that the confirmed genetic diagnosis of a medical condition does not obviate the possibility of developing a clinically similar but genetically different condition. Methods: The index case described is a member of a traveller family known to carry a novel insulin receptor [INSR] mutation (p.(Met1180Lys),c.3539T>A). This mutation is transmitted in an autosomal dominant fashion and causes insulin resistance, hyperlipidaemia, hirsutism and hypertension due to high levels of circulating insulin. In adults, this leads to hyperglycaemia due to insulin resistance and a Type 2 diabetes phenotype. The index case was identified on sibling screening as other members of the family had presented with hypoglycaemia in infancy. Previous investigations at time of genetic diagnosis did not suggest dysglycaemia. The boy then presented at 2 years of age with a one-week history of polyuria and polydipsia, vomiting and abdominal pain.

Results: Biochemistry at presentation was consistent with Diabetes ketoacidosis [pH 7.22, glucose $26.5 \mathrm{mmol} / \mathrm{L}$, ketones $>5$ ]. He was commenced on the DKA protocol but required minimal insulin for correction suggested that he was sensitive to insulin administration despite his underlying diagnosis. Diabetes autoantibody screen was positive for IA-2 and ZnT8 [confirming autoimmune Type 1 diabetes]. A Diabetes polygenic score which ascertains the genetic risk of developing Type 1 diagnosis by screening his HLA haplotype confirmed a $>99 \%$ likelihood of having Type 1 diabetes. He is currently managed on an insulin pump and dietary measures.

Conclusion: There are no published cases of co-existence type 1 and type 2 diabetes due to genetic causes. Recent studies in obesity suggest that acquired insulin resistance accelerates progression to type 1 diabetes in high-risk individuals with islet autoimmunity. His long-term outcome is also unclear as insulin resistance is linked with early onset of complications.

\section{Management Of Postpartum Haemorrhage During The Daytime And Night Time}

Kamath P, Downey A, Rooney DJ, Ravikumar N, Gannon M, Thomas S, Almshwt M 
Dept. of Obstetrics and Gynaecology, Midlands Regional Hospital, Mullingar

\section{$\mathrm{DML} / \mathrm{UCD}$}

Background: The National Perinatal Epidemiology Centre (NPEC) has shown that the rate of postpartum haemorrhage (PPH) in Ireland increased from $1.5 \%$ in 1999 to $4.1 \%$ in 2009 . The rate of blood loss exceeding 2.5 litres, has increased from 2.34 per 1000 maternities in 2011 to 3.14 per 1000 maternities in 2017. Differences have been demonstrated in obstetric management and outcomes during the day or night. Aim: Our aim was to assess for differences in PPH rates during the day and night time in Mullingar Regional Hospital and use the findings to investigate and improve existing PPH management protocols in the unit. Study Design \& Methods: We conducted a retrospective chart analysis of all cases of PPH from January to June 2019 through the local HIPE system. PPH for all deliveries was defined as blood loss over $500 \mathrm{ml}$. Minor PPH was defined as $500 \mathrm{ml}-1000 \mathrm{ml}$, moderate as $1000 \mathrm{ml}$ $2000 \mathrm{ml}$, and severe as greater than $2000 \mathrm{ml}$.

Findings: There were 986 deliveries from January to June 2019. The $\mathrm{PPH}$ rate was $8.1 \%$. The overall rate of PPH for LSCS was $6.2 \%$ and for vaginal deliveries was $9 \%$. The $\mathrm{PPH}$ rate during the day was $6.2 \%$ and during the night was $9.6 \%$. For severe $\mathrm{PPH}$, there were 5 cases during the day and 4 cases at night. 2 cases required balloon insertion at night and there were no hysterectomies or PPH greater than $2.5 \mathrm{~L}$. The blood transfusion rate during the day was $0.7 \%$ and $1.6 \%$ at night.

Conclusion: This study showed that the incidence of PPH at is higher at night compared to day. The rate of major obstetric haemorrhage as defined by NPEC is in line with the national standard.

\section{Outcomes Of Sequential Pneumatic Compression In Critical Limb Ischemia}

Wang T, Sultan S

Dept. of Vascular and Endovascular Surgery, University Hospital Galway, National University of Ireland, Galway

\section{WNW/NUIG}

Introduction: Critical limb ischemia (CLI), the severe obstruction of peripheral vasculature, presents in elderly patients who often have comorbidities that prevent surgical intervention. Sequential pneumatic compression (SPC) has been suggested to reduce amputations, relieve pain, and promote wound healing by increasing arterial blood flow to distal limbs. Objective: This study examines patients presenting with CLI that were prescribed SPC using the device ArtAssist by comparing the outcomes of patients who acquired the device with those who did not. The outcome measures were revascularisation, minor amputation (below ankle), major amputation (above ankle), and all-cause mortality.

Methods: A retrospective chart review was conducted using Vascubase and Evolve software. Inclusion criteria for the experimental group were patients prescribed SPC who used ArtAssist from 2008 to present in Galway University Hospital $(n=395)$. Control group consisted of patients who were prescribed SPC but did not acquire ArtAssist ( $n=71)$. Patient demographics and outcomes were collected and statistically analysed using Chi-square test on SPSS.

Results: In the experimental group, 46 (9.9\%) patients underwent revascularisation, $23(5.8 \%)$ had minor amputations, $41(10.4 \%)$ had major amputations, and 117 patients $(29.6 \%)$ passed away. In the control group, 6 patients $(8.6 \%)$ underwent revascularisation, $4(5.6 \%)$ had minor amputations, $11(15.5 \%)$ had major amputations, and $36(50.7 \%)$ patients passed away. No statistically significant difference was found in the number of revascularisations $(p=0.431)$, the number of minor amputations $(p=0.95)$, or number of major amputations $(p=0.208)$ between the intervention and control group. However, there was a statistically significant difference in all-cause mortality $(\mathrm{p}=0.0005)$.
Conclusion: A statistically significant reduction of all-cause mortality was found in the experimental group, though no significant difference was found in the number of revascularisations or amputations. These results show that SPC is correlated with the decrease of all-cause mortality in patients with CLI.

\section{Reference:}

Zaki M, Elsherif M, Tawfick W, El Sharkawy M, Hynes N, Sultan S. (2016). The Role of Sequential Pneumatic Compression in Limb Salvage in Non-reconstructable Critical Limb Ischemia. European Journal of Vascular \&Endovascular Surgery. 51(4): 565-71. DOI: https://doi.org/ 10.1016/j.ejvs.2015.12.025.

\section{Wellness \& Burnout in the Irish Surgical Cohort}

O’Donnell JPM, Murphy D, Sugrue RM, Cinelli D, Kelly J Plastic \& Reconstructive Surgery Dept., Galway University Hospital

\section{WNW/NUIG}

Introduction: Burnout is a growing epidemic threatening healthcare systems globally. Irish national awareness campaigns have highlighted pitfalls and protective factors. Internationally, burnout in US physicians is up to $51 \%$. Current guidelines are generated from US studies mostly constructed around healthcare systems dissimilar to that experienced by Irish surgeons undergoing incomparable training schemes. Therefore, we challenge whether international data is reproducible and relevant in the Irish healthcare system.

Methods: This prospective cohort study of National Surgical Trainees used the Maslach Burnout Index (MBI) to validate the degree of burnout. Irish-specific risk and protective factors were quantified with a standardised Likert Scale. All responses were then reviewed by two independent reviewers to compare readings of the MBI. Demographic analysis was conducted. Microsoft Excel \& SPSS were used as data processing tools.

Results: Fifty-seven (57) Irish practising surgeons participated. 33\% of surgeons in Ireland are burned out. A subanalysis shows the rate of burnout for SHO and SPR was $42 \%$ and $31 \%$ respectively Protective factors most associated were; older age and a family. Daily workload, lack of resources, and 'on-call' commitments were the greatest risk factors. Depersonalisation was highly experienced across all groups, regardless of burnout status. Both groups were only "somewhat likely" to recommend a career in surgery to family or friends.

Conclusion: This is the largest prospective cohort assessment of burnout in surgical trainees in Ireland. It shows an increase in burnout rate both nationally and internationally. The conclusive protective factors show be introduced into awareness campaigns to provide insight for Irish surgeons, and improve patient and doctor care.

\section{Evaluation Of A Novel Diagnostic Criteria For Tarsal Tunnel Syndrome}

Conway $\mathrm{S}^{1}$, Downey $\mathrm{C}^{1}$, Moroney $\mathrm{P}^{1}$, O’Brien $\mathrm{CP}^{2}$
Cappagh National Orthopaedic Hospital
, Sports Surgery Clinic

\section{DNE/RCSI}

Introduction: Tarsal Tunnel syndrome (TTS) is caused by compression of the posterior tibial nerve or its branches. This can present with a mixed picture of pain and paraesthesia as well as muscle weakness in the distribution of the posterior tibial nerve. There are no gold-standard diagnostic criteria for TTS. It is frequently misdiagnosed as plantar fasciitis.

Aims: The aim of this study was to investigate the efficacy of a novel diagnostic criteria for TTS which has been developed at a national referral centre. Secondary objectives:

(1) To assess the diagnostic yield of a novel diagnostic criteria for TTS 
(2) To assess the outcomes of patients diagnosed using this methodology treated with injection therapy.

Materials and Methods: A clinical diagnosis of TTS was made for each patient by history and clinical examination. Any history of trauma or surgery to the lower limb and any history of thyroid disease, diabetes or other medical conditions associated with peripheral neuropathies was recorded.

A 12 parameter electrodiagnostic test was then carried out. Distal latency and amplitude were measured for tibial motor supply to abductor hallucis $(\mathrm{AH})$ and abductor digiti quinti (ADQ). Distal latency and amplitude were also measured for sensory supply to medial and lateral plantar and calcaneal nerves. Electromyography to $\mathrm{AH}$ and $\mathrm{ADQ}$ muscles was also performed.

Clinical records were examined for follow up and outcomes of these patients.

Results: The results indicate a significantly increased diagnostic yield for TTS $(3.3 \%)$ compared to current methods $(0.5 \%)$. The patients had a mixed picture of positive electrophysiological tests with the average being 5 positive tests. Most patients have a history of ankle injury or ankle surgery. There is a significant positive response to injection therapy $(64 \%)$.

Conclusions: This 12-parameter diagnostic criteria are viable diagnostic criteria for TTS, improving the diagnostic yield from current standard methods.

\section{"Paperwait"- A Clinical Audit On The Hidden Time Trap Of Paper Charts}

Glancy C, Guerandel A, Day L, McCarthy N, Gildea J

Dept. of Psychiatry, St Vincent's University Hospital, Dublin

\section{DML/UCD}

Background: A great deal of time is expended by healthcare professionals searching for patient information in cumbersome medical charts. A large amount of patient information is currently locked in paper format, accessible only in certain locations and susceptible to risk of loss or damage. Aims: We sought to compare time spent obtaining information from paper charts versus online counterparts. In doing this we wished also to highlight any disparities in information available from paper or online databases.

Methods: Online duplicates of medical files were created and stored on an encrypted USB. Direct comparison of patient's online/physical files was used to reduce risk of bias and to highlight any discrepancies. Data duplicated included recent blood results, healthcare correspondence and ECGs and was compiled from an online hard drive and hospital databases. Results: Thirty (30) medical charts were duplicated into online versions and directly compared. The total time to search through all paper charts was 49 minutes. The mean time to check a paper file was 1.38 minutes and the longest length of time was 3.56 minutes. In comparison the total time spent obtaining data from 30 online files was 4 minutes. The mean time taken to check an online file was 8 seconds and the longest time spent on any one file was 17 seconds. Disparities in information available were also noted during the search. For example, some paper charts were missing blood results etc.

Conclusions: This study demonstrated a significant time difference in time spent searching for information in paper charts versus online folders. This has implications on time management for healthcare professionals. The study also highlighted incongruity in information available through different mediums which may impact the management of patients and their conditions.

\section{A Case Report Of Exomphalos Major}

Brennan E, Fitzsimons J

General Paediatrics Dept., Temple Street University Hospital, Dublin

\section{$\mathrm{DML} / \mathrm{UCD}$}

Introduction: Exomphalos major is a rare abdominal wall defect $>4 \mathrm{~cm}$ with herniation of abdominal organs including the stomach, liver, pancreas, spleen and intestines. The incidence is estimated at 1 in 6000 live births. (1)

Case report: A 1-year old boy with an Antenatal diagnosis of exomphalos major made at routine scanning at 15 weeks was delivered via emergency $\mathrm{C}$-section at $37+6$ weeks. Birth weight was $1.75 \mathrm{~kg}$, APGAR was 5,7 at 1 and 5 minutes and he required IPPV and intubation at 12 minutes of life due to poor respiratory effort. He was transferred from a maternity hospital to Temple Street PICU due to respiratory failure on a background diagnosis of pulmonary hypoplasia. He had a prolonged 4 months stay in PICU for intubation + ventilation. His CT AP at 2 days old demonstrated a giant exomphalos which contains spleen, pancreas, stomach and large/small bowel excluding the rectum/sigmoid colon, kidneys and urinary bladder which are in their normal anatomical location. He was initially managed conservatively; eschar and epithelisation initially dressed with silver sulfadiazine and gauze. His exomphalos at 12 months old has fully epithelialized. His antenatal microarray was normal however he was diagnosed with congenital hypothyroidism via newborn screening assessment and is currently being managed on $37.5 \mathrm{mcg}$ of levothyroxine. Nutrition and growth have proved challenging, He is currently on patient specific parenteral nutrition via $\mathrm{NJ}$ feed and at 12 months old weighs $7 \mathrm{~kg}$.

Discussion: Infants with exomphalos major usually require prolonged hospitalization for associated genetic abnormities, pulmonary hypoplasia and complex nutritional needs. In comparison to Gastroschisis, Chromosomal abnormalities have been reported in $10-12 \%$ of the neonates with exomphalus and $30 \%$ of the fetuses with exomphalos. (2). Trisomy 18 and trisomy 13 are the most common chromosomal abnormalities found (3). In this case, there was normal antenatal karyotyping however congenital hypothyroidism was diagnosed on newborn heel prick screen. This patient has been an inpatient for his 12 months of life, Intensive daily MDT input is crucial in management these patient's complex needs.

References:

1. Giant Omphalocele: current perspectives. Mack.AJ (1) 18th july 2016 volume 2016 pages 33-39. Research and Reports in Neonatology.

2. Omphalocele and Gastroschisis: An 18-year review study. P. Hwang (1) Genetics in medicine 2004 232-236

3. Fetal exomphalos and chromosomal defects: relationship to maternal age and gestation Snijders. Ultrasound Obstet Gynecol (1995), pp. 250255

Changing Profile Of Patients Admitted To Large Nursing Homes. A Four Year Audit Of 7 Facilities Covering Over 600 Beds Across Ireland

Weiner-Gorzel K, Maher RL, Patindol LS, Subari SR, Power D Med El, Fairview, MMUH, Dublin

\section{$\mathrm{DML} / \mathrm{UCD}$}

Background: To meet increasing demand, a significant number of private nursing homes have recently been developed, many as part of larger groups of homes run under the same corporate governance structure. One such group, operates 7 facilities across Ireland. As part of their quality protocol, patient data are collected monthly and presented to a quality and safety committee.

Methods: We reviewed 4 years of monthly data (January 2014 to December 2017) covering 600 beds across 7 sites, including measurements on patient's age, length of stay, dependency level, dementia diagnosis, falls rate, and mortality rate. We compared our data over time and between urban and rurally situated facilities. 
Results: Mean patient age on admission over the 4 years of the study was 80.2 years. Over the 4 years of the study, the mean admission age remained unchanged. Mean length of stay (AvLOS) of 33.2 months over the 4 years but decreased from 34.7 years in 2014 to 31.3 years in 2017 . Interestingly, dementia was reported in $78.5 \%$ of patients in the urban nursing homes versus $33.2 \%$ in rurally located facilities $(p<0.05)$. This compares to a national statistic of $36 \%$. Over the 4 years of the study, high/maximum dependency rates increased across all sites but was significantly higher in the urban versus the rurally located homes $(82 \%$ vs $44 \%, \mathrm{p}<0.03$ ). Falls rate dropped over the study period to less than 0.01 per occupied bed per night.

Discussion: This provides a novel insight into the changing profile of patients admitted to a large nursing home group over the last number of years. The observed falling AvLOS possibly represents improved homecare services delaying the requirement for residential care. Similarly, the significantly lower dementia rates noted in rural facilities may represent a lack of such home services.

\section{A Twenty-Year Review Of Endoprostheses In Lower Limbs In A National Referral Centre}

Conway S, Murphy EP, O'Toole G, Molloy A

Dept. of Orthopaedic Medicine, Cappagh National Orthopaedic Hospital, Dublin

\section{DNE/RCSI}

Introduction: Limb salvage procedures have become more prevalent due to successful advances in the treatment of bone tumours. While limb amputation was previously offered, the gold standard now is to discuss cases at a tumour multidisciplinary meeting where a treatment strategy is mapped out. Limb function is a major concern in these mega en bloc resections with limb salvage reconstruction. Endoprostheses are devices to reconstruct large skeletal deficits.

Aims: To complete a twenty-year review of the outcomes of lower limb salvage reconstruction with endoprostheses at Cappagh National Orthopaedic Hospital (CNOH).

Secondary objectives:

(1) To describe any complications associated with surgical treatment.

(2) To describe the epidemiological trends of patients who underwent surgical limb salvage procedures

(3) To describe the modes of failure/revision of the implants

Materials and Methods:

A retrospective review was conducted of a single national referral centre over a 20-year period. Epidemiological data including age at time of diagnosis, surgery, time to revision/complications and mortality were recorded. Radio-graphical records and pathology reports were accessed for follow up and diagnosis.

Inclusion criteria:

Bone tumour with reconstruction using an endoprosthesis in the lower limb. Exclusion criteria:

Upper limb bone tumours

Endoprostheses used for non-bone tumours

Tumours not treated with limb salvage procedures.

Results: A total of 107 patients were included in the study. The locations for prosthesis included were distal femoral (64), distal femoral and proximal tibial (14) proximal tibial (17), proximal femoral (8) and total femoral (3). The primary indications for endoprosthesis insertion were osteogenic sarcoma (61), Ewing's sarcoma (10) and chondrosarcoma (11).

There were 55 cases requiring further procedures $(51 \%)$. The reasons for further surgery included infection, tumour recurrence and failure of prosthesis.

Conclusions: This study accurately describes the trends in limb salvage surgery for a national referral centre over a 20 -year period.
To Investigate The Relationship Between Overactive Bladder And Pelvic Organ Prolapse

Daly R, O'Sullivan O

Dept. of Urogynaecology, Cork University Maternity Hospital $\mathrm{S} / \mathrm{UCC}$

Introduction: Overactive bladder $(\mathrm{OAB})$ is a highly prevalent disorder with a potentially profound impact on quality of life. While it's precise etiology remains elusive, $\mathrm{OAB}$ frequently co-exists with pelvic organ prolapse among the older female population. Treatment of prolapse often results in improvement in OAB symptoms. However, the nature of the relationship between these two conditions is unclear. The purpose of this study was to determine the prevalence of prolapse among women with overactive bladder, and to further contribute to the understanding of the relationship between these two conditions.

Method: This retrospective, single institution study included women who were referred for urodynamic testing. Measured outcomes included urinary symptoms at presentation, prolapse compartment and grade on pelvic examination, and urodynamic test results.

Results: 286 patient charts were reviewed. $219(76.6 \%)$ had a diagnosis of OAB (69.4\% OAB wet, $30.6 \%$ OAB dry). Mean age was 55.6 years $(\mathrm{SD}=14.3) .120(54.8 \%)$ were found to have a coexisting prolapse on pelvic examination (73.3\% anterior, $39.2 \%$ posterior, $23.3 \%$ apical wall prolapse.) No significant correlation was demonstrated between $\mathrm{OAB}$ and the presence of an anterior, posterior or apical wall prolapse $(p=0.474$, $0.271,0.091$ respectively). There was no significant difference regarding urodynamic parameters for women with $\mathrm{OAB}$ and prolapse, versus those without prolapse. Occult stress urinary incontinence was recorded in $72.6 \%$ of patients with $\mathrm{OAB}$ and coexisting prolapse.

Conclusion: Pelvic organ prolapse is prevalent among women with $\mathrm{OAB}$, in particular anterior compartment prolapse. As the relationship between these conditions remains poorly understood, women undergoing treatment for prolapse should be counselled on the risk of persistent $\mathrm{OAB}$ symptoms postoperatively, as well as the development of de novo stress urinary incontinence, which may improve management of patient expectations.

A Case Of Venoarterial-Extracorporeal Membrane Oxygenation Therapy For Cardiogenic Shock Secondary To A Phaeochromocytoma

Griffin S, Conrick-Martin I

Dept. of Critical Care Medicine \& Anaesthesiology, Mater Misericordiae University Hospital, Dublin

\section{$\mathrm{DML} / \mathrm{UCD}$}

Background: Phaeochromocytomas are rare, catecholamine-secreting, neuroendocrine tumours which present in approximately $3 \%$ of patients with Neurofibromatosis Type 1 (NF1).1 Catecholamine-crisis can result in multiorgan dysfunction including an acute stress cardiomyopathy. Venoarterial - Extracorporeal Membrane Oxygenation (VA-ECMO) salvage therapy for cardiogenic shock secondary to phaeochromocytoma has rarely been reported. 2

Case-Report: A 30-year old female presented to Mater Misericordiae University Hospital (MMUH) with right upper-quadrant pain and was found to be hypertensive, tachypnoeic and tachycardic on a background of NF1 and a one-year history of palpitations \& anxiety. Initial bloodwork was notable for severe lactic acidosis with elevation in d-dimers \& high-sensitivity troponin. Electrocardiogram showed sinus tachycardia without ischaemic changes.

CT Pulmonary-Angiogram out-ruled pulmonary embolism. However, bibasal consolidation was demonstrated; clinically consistent with evolving refractory pulmonary oedema, which ultimately necessitated mechanical ventilation. CT Abdomen was significant for a $6 \mathrm{~cm}$ right-adrenal 
mass, which had undergone haemorrhagic transformation and ruptured. Bedside Transthoracic Echocardiogram demonstrated severe left ventricular systolic dysfunction with circumferential basal hypokinesis.

Metoprolol was administered due to persistent tachycardia $>180$ following intubation. Hypotension ensued requiring boluses of Metaraminol for haemodynamic stability. Phentolamine was subsequently delivered with almost immediate loss of output and PEA cardiac arrest evolved. Return of spontaneous circulation was achieved after 4 minutes of Advanced Cardiac Life Support. Peripheral VA-ECMO was initiated upon transfer to ICU.

VA-ECMO decannulation occurred 5 days later following recovery of myocardial function. Haemodynamic stability was achieved with a progressive $\alpha$-and subsequently $\beta$-blockade with Phenoxybenzamine and Propranolol respectively. An elective laparoscopic adrenalectomy was performed 8 weeks later without complication.

Discussion: Phaeochromocytoma should be suspected in patients presenting with hypertension-associated cardiogenic shock and abdominal imaging obtained. Treatment of catecholamine-induced stress cardiomyopathy requires careful introduction of an $\alpha$-blockade followed by a $\beta$ blockade if necessary. 3 The rapid reversibility of phaeochromocytomainduced cardiogenic shock makes such patients good candidates for VAECMO.

\section{References:}

1. Gruber L et al. Pheochromocytoma and paraganglioma in patients with neurofibromatosis type 1. Clinical Endocrinology. 2016;86(1):141-149.

2. Hekimian $\mathrm{G}$ et al. Extracorporeal membrane oxygenation for pheochromocytoma-induced cardiogenic shock. Annals of Intensive Care. 2016;6(1).

3. Casey R et al. Management of an acute catecholamine-induced cardiomyopathy and circulatory collapse: a multidisciplinary approach. Endocrinology, Diabetes \& Metabolism Case Reports. 2017.

Functional Capabilities After Metatarsal Phalangeal Joint Arthrodesis Using A Locking Plate, Compression Screw Construct

\section{Murphy FM, Curran MG, Murphy EP, Fenelon CF, Kearns SR Orthopaedic Dept., Galway University Hospital Group}

\section{WNW/NUIG}

Background: Arthrodesis of the first metatarsal phalangeal joint (MTPJ) is a widely utilized surgical procedure for a wide array of metatarsal pathologies due to its relative safety and efficacy. First MTPJ arthrodesis is commonly used to surgically treat hallux valgus, painful hallux rigidus and patients that are affected by degenerative conditions. This study will explore the functional limitations following first MTPJ arthrodesis, overall satisfaction and patient's abilities to achieve ADLs postoperatively.

Methods: This was a retrospective study assessing functional limitations as well as footwear and lifestyle restrictions using a questionnaire. The study recruited 103 participants who underwent an isolated first MTPJ arthrodesis under the care of a single surgeon. Pre and post-operative patient reported outcome measures were recorded. The American Foot and Ankle Outcome Score (AFAOS) and the Manchester-Oxford Foot Questionnaire (MOxFQ) were also used to further examine functional status. The hallux valgus angle (HVA) and inter metatarsal angle (IMA) were compared using preoperative and postoperative weight-bearing anterior-posterior foot radiographs. Successful fusion was also recorded. Complications were recorded and are discussed in detail.

Results: Successful fusion was confirmed in $97.1 \%$ of participants using their post-operative $\mathrm{x}$-ray 6 weeks after the procedure. There were no serious postoperative complications following the procedure. Two patients had delay to fusion. One patient required a revision arthrodesis after a non-union. $82.5 \%$ of patients were able to return to wearing nonadaptive footwear with a decrease in orthotic therapy also noted. $63.1 \%$ of patients were able to participate in dancing. The mean reduction in HVA was 21.78 degrees with a reduction of IMA angle by 6.84 degrees.
Conclusion: This study demonstrates the successful use of a compression screw/locking plate construct for arthrodesis of the first MTPJ. The study quantifies the reduction in angles recorded, outlines respective complications while providing qualitative information with respect to ADLs and QOLs.

\section{Evaluating The Quality Of Documentation Of Social History In Paediatric Medical Admission Notes At A Regional Paediatric Centre}

Hannon $\mathrm{J}^{1}$, Foley $\mathrm{D}^{1}, \mathrm{O}^{\prime}$ Gorman $\mathrm{C}^{1,2}$, Gallagher $\mathrm{S}^{1}$, Murphy $\mathrm{AM}^{1}$ Dept. of Paediatrics, University Hospital Limerick ${ }^{1}$ Graduate Entry Medical School (GEMS), University of Limerick ${ }^{2}$

MW/UL

Background and Aims: The psychosocial history is long established as an area of importance in patient care. Our institution serves a catchment area of over 100,000 children less than 16 years of age, from diverse backgrounds.

Our aim was to assess the content of psychosocial history documentation in medical admission notes, highlighting areas for education and improvement, so as to identify patients living in high-risk situations, and adjust care appropriately, including establishment of better local social supports and services.

Methods: This study involved concurrent analysis of 75 paediatric medical admission notes, taken in even distribution from two Paediatric wards and examined over a three-week period. Data on documentation of social history was collected using a standardized excel data collection tool and analysed.

Following an education session for NCHDs, a second audit of 20 charts was conducted.

Results: Initially, documentation of any social history occurred in 31 of 75 charts $(41 \%)$. Common areas documented were parental marital status (29/75: 38\%), household constituents (19/75: 25\%) and school attendance $(12 / 75: 16 \%)$. Areas which have implications for disease progression, including smoking status and patient accommodation type were recorded in 2/75 (2.6\%) and 0/75 (0\%) of charts respectively.

On re-assessment, documentation of any social history occurred in $95 \%$ (19:20) of charts, showing an improvement of $53.7 \%$, with parental marital status documented in $85 \%$ and household constituents in $95 \%$. School attendance showed a 54\% improvement in documentation; however, smoking status and accommodation type remained poorly documented, with an improvement of $22.4 \%$ and $0 \%$ seen respectively.

Conclusion: There is significant variability in the quality of social history-taking in our department. Overall, documentation of psychosocial circumstances is poor.

Research regarding the reasons for this and education amongst clinicians on the need for detailed psychosocial record-taking - thus allowing us to tailor care to individual patients- is required.

\section{POSTER PRESENTATIONS}

An Audit On The Prescribing Of Oral Penicillin Antibiotics In The Paediatric Population In The Republic Of Ireland

Browne D, O'Brien D

Dept. of Microbiology, Mercy University Hospital, Grenville Place, Cork

$\mathrm{S} / \mathrm{UCC}$

Introduction: Antimicrobial resistance poses a significant threat to our health. A key element of antimicrobial stewardship is the use of appropriate dosing ${ }^{1}$. Studies ${ }^{2,3}$ in the UK have identified that the paediatric population are often prescribed lower than the recommended dosage of penicillin antibiotics. This research aimed to assess adherence to 
nationally determined antimicrobial dosing guidelines ${ }^{4}$ by examining dosage with respect to the patients age. Frequency of dosing and duration of treatment was also examined.

Method: Data for this Clinical Audit was collected from fifteen community pharmacies in the Munster region, over a two-month period using a data collection form. A total of 1177 prescriptions were included in the study. The criteria for selection of prescriptions were: Patients aged between 1 month and 17 years at the time of data collection; who were prescribed a penicillin antibiotic (amoxicillin, phenoxymethylpenicillin, flucloxacillin or co-amoxiclav) used to treat an acute infection.

Results: In general, $51.83 \%(\mathrm{n}=610)$ of patients were prescribed the recommended dose of penicillin antibiotic, $48.17 \%$ were prescribed the incorrect dose or frequency or both. In the amoxicillin group, $66 \%$ of patients were prescribed lower than the recommended dose. A significant number of patients in the flucloxacillin and phenoxymethylpenicillin groups were prescribed the incorrect frequency of dosing, $43.88 \%$ and $21.9 \%$ respectively, in many cases the antibiotic was prescribed three times daily dosing rather than the recommended four times daily dosing. In contrast, in the co-amoxiclav groups, (Augmentin Duo and Augmentin Pediatric) the majority of patients were prescribed the correct dose and frequency, $92 \%$ and $91 \%$ respectively.

Conclusion: This audit clearly shows that penicillin antibiotics are not being prescribed in accordance with recommended guidelines. Action needs to be taken to remind clinicians of the importance of prescribing antibiotics based on updated guidelines to minimise the risk of antimicrobial resistance.

\section{References:}

Gerding DN. The search for good antimicrobial stewardship. The Joint Commission journal on quality improvement. 2001;27(8):403-4.

Saxena S, Ismael Z, Murray ML, Barker C, Wong ICK, Sharland M, et al. Oral penicillin prescribing for children in the UK: A comparison with BNF for Children age-band recommendations. Br J Gen Pract. 2014;64(621).

Rann O, Sharland M, Long P, Wong ICK, Laverty AA, Bottle A, et al. Did the accuracy of oral amoxicillin dosing of children improve after British National Formulary dose revisions in 2014? National crosssectional survey in England. Vol. 7, BMJ Open. 2017.

HSE. HSE Antibiotic Prescribing Website [Internet]. 2018 [cited 2018 Nov 26]. Available from: https://www.hse.ie/eng/services/list/2/gp/ antibiotic-prescribing/

\section{An Audit Of Urine Collection Techniques In University Hospital Limerick Of Infants And Children With Suspected Urinary Tract Infections}

Morrissey B, Thompson C, Twomey J

Dept. of Paediatrics, Limerick

\section{MW/UL}

Urine collection methods currently employed in University Hospital Limerick include both bag collection and clean catch collection.

This audit examines the current practices, and preferred methods of urine collection in University Hospital Limerick, in both the Emergency Dept and in the paediatric ward setting. Data was obtained retrospectively from paediatric records. Information was obtained, including age and gender, together with presenting complaint. Method of urine collection employed was noted, as was microscopy result. The number of urine samples taken was further analysed, and the number of false positive results and false negative results documented.

Initial results demonstrate that bag collection of urine is preferentially used in pre-continent infants and children $(10 \%)$ versus clean catch samples in continent children (100\%) in UHL. All infants with a positive bag sample, had a further clean catch performed so as to confirm diagnosis. False positives were noted in approximately $40 \%$ of those with bag samples positive for leukocytes.
As such, it is evident that while the use of urine bag samples is convenient, it is less accurate than clean catch sampling and may result in delay in initiation of treatment or inappropriate treatment of UTI.

\section{Neuroleptospirosis In A Returned Traveller}

O'Farrell R, Burke O, Costello R, Moynan D, Raftery A, McNally C Dept. of Infectious Disease, Beaumont Hospital, Dublin DNE/RCSI

Leptospirosis is a zoonotic disease prevalent worldwide. The clinical presentations are diverse, ranging from undifferentiated fever to fulminant Weil's disease. We highlight the case of a young man who returned from a 12-day trip from Northern Thailand, in the Chiang Mai region with a fever of unknown origin. Several days post return, he presented to AE reporting recurrent fevers and flu like symptoms including headache, chills and myalgia. He reported exposure to outdoor water sources in rural Thailand and animal contact included bathing with elephants. $\mathrm{He}$ had also engaged in a water sports obstacle course in Australia prior to his Thailand travel. There was no evidence of high-risk behaviour or risk factors for blood borne viruses identified. On admission nil neurologic deficits were noted and his temperature was $38.6^{\circ} \mathrm{C}$.

Laboratory investigations showed liver function tests were within normal range. Renal profile demonstrated a mild AKI. C-reactive protein was 253. Initial extensive investigations proved negative. There was no serological evidence of recent primary EBV, CMV or toxoplasmosis infection. Serology for HIV, treponema pallidum, dengue, salmonella, malarial parasites and hepatotropic viruses were negative. The CT-brain performed was normal. A clinical deterioration occurred in the following days and a lumber puncture was performed. Evidence of aseptic meningitis was confirmed on CSF results. Further testing revealed IgM positive serology for leptospirosis, confirming a case of neuro-leptospirosis meningitis.

Discussion: This case illustrates the rarity of CNS involvement of leptospirosis. Plausible environmental and occupational exposure should raise suspicion of leptospirosis infection and should be an important differential diagnosis for many tropical infections. Although this gentleman did not show any of the other more common symptoms of leptospirosis, such as jaundice, thrombocytopenia, or abnormalities in hepatic functions, it is important to consider leptospirosis in returned travellers and identify potential CNS complications, to facilitate prompt antibiotic use.

Screening And Detection Of Connective Tissue Disease Associated Pulmonary Arterial Hypertension In Systemic Sclerosis Patients

Dr. Sarah Faulkner ${ }^{1}$, Dr. Miriam O’Sullivan ${ }^{1}$, Dr. Carmel Silke ${ }^{1,2}$, Dr. Bryan Whelan ${ }^{1,2}$, Dr. Tauseef Ghaffar ${ }^{1}$

The North Western Rheumatology Unit ${ }^{1}$, Our Lady's Hospital, Manorhamilton, Co Leitrim, Ireland

Dept of Medicine ${ }^{2}$, National University of Ireland Galway

\section{WNW/NUIG}

Introduction: Systemic sclerosis (SS) is an autoimmune, rheumatic disease that affects the body by hardening connective tissue. Scleroderma is the classical skin feature associated with it. "Sclera" means hard and "derma" means skin so literally scleroderma means hard skin.

Pulmonary hypertension is high blood pressure $(>25 \mathrm{mmHg}$ at rest) in the blood vessels of the lungs.

Prevalence is about $10 \%$ in systemic scleroderma patients. Prevalence is actually higher (15\%) in limited scleroderma but, these patients have later onset PAH.

Aim: The aim is to examine how the Rheumatology service performs compared to the ACR guidelines in terms of screening for Pulmonary Hypertension with Echocardiogram, PFTs, and pro-BNP.

ACR Guideline: It was recommended that transthoracic echocardiogram and PFTs should be performed annually. The full screening panel (transthoracic echocardiogram, PFTs, and measurement of NT-proBNP) 
should be performed as soon as any new signs or symptoms are present. (1)

Role of Pro BNP: A cut-off value at or $>395 \mathrm{pg} / \mathrm{mL}$ of N-TproBNP strongly supports the diagnosis of SSc-PAH in patients where this diagnosis is suspected, with a positive predictive value of $95 \%$. it is clear that the positive and negative predictive values are similar to those observed with echocardiography. (2)

Method: Fifteen (15) patients with SS attending the Rheumatology service were chosen. We looked retrospectively at when their last PFTs, Echocardiogram, and pro-BNP tests were done.

\section{Results:}

$80 \%$ of these patients followed the recommended guidelines for annual Echocardiogram while $60 \%$ followed the guidelines for annual PFTs.

$100 \%$ had PFTs and echocardiogram done within the last two years.

Only $27 \%$ of patients had a record of having a pro-BNP done.

Conclusion/Recommendations:

1. A page in every scleroderma patient chart that documents when the last $\mathrm{ECHO}, \mathrm{PFTs}$, and BNP were done.

2. Baseline BNP for scleroderma patients when ordering their first ECHO. Repeat if symptomatic.

\section{References:}

1. Khanna, Dinesh, et al. "Recommendations for Screening and Detection of Connective Tissue Disease-Associated Pulmonary Arterial Hypertension." Arthritis and Rheumatism, vol. 65, no. 12, 2013, pp. 3194-201, www.ncbi.nlm.nih.gov/pubmed/24022584, 10.1002/ art.38172. Accessed 26 Sept. 2019.

2. Williams, Mark H., et al. "Role of N-Terminal Brain Natriuretic Peptide (N-TproBNP) in Scleroderma-Associated Pulmonary Arterial Hypertension." European Heart Journal, vol. 27, no. 12, 27 Apr.

Rational Use Of Shoulder MRI In The Private Setting: How Often Does The MRI Actually Influence Clinical Management?

Devlin R, Delaney RA

Dublin Shoulder Institute and Royal College of Surgeons in Ireland, Dublin

\section{DNE/RCSI}

Aim: The aim of this study was to determine the difference in proportion of shoulder MRIs that influence the management plan of shoulder patients based on whether MRI was ordered by a shoulder specialist orthopaedic surgeon or primary care provider prior to referral to a specialist.

Methods: This observational analytical study was conducted in a private practice setting. Data were obtained from 153 MRIs performed on 151 patients. 77 MRIs were ordered by a specialist shoulder surgeon and 76 by a primary care provider (general practitioner, non-operative sports medicine physician or physiotherapist).

Results: Specialist-ordered MRIs influenced patient management significantly more often than primary care-ordered MRIs ( $82 \%$ vs. $22 \%$, p < 0.001 ). $54 \%$ of referral letters from primary care providers to the specialist did not have documentation of a physical examination, yet an MRI had been ordered. The most common diagnoses for primary care-ordered MRIs which did not have influence on patient management were subacromial bursitis and adhesive capsulitis.

Conclusion: With less than $25 \%$ of primary care-ordered shoulder MRIs influencing clinical management, questions must be raised about the indications for MRI. Greater than $50 \%$ of referrals contained no documented physical examination, suggesting that MRI is being relied upon for assessment. If access to private MRI was to be rationalised, perhaps shoulder specialist-ordered CT and X-ray could be covered by insurance providers. Currently they are not covered in our system, yet are more likely to influence clinical management than primary care-ordered MRIs, which are currently covered by insurance without restriction on indications.
Efficacy And Adverse Events With The Use Of Tranexamic Acid In The Hip Fracture Population In A University Teaching Hospital

Scanlon E, Geary E, Keeling P, Hurson C, Doyle R, O'Hanlon S, Curtin P Dept. of Orthopaedics, St Vincent's University Hospital, Dublin

$\mathrm{DML} / \mathrm{UCD}$

There is currently an international and national focus on methods to optimize the care of patients with hip fractures. While there is little literature supporting it, perioperative tranexamic acid (TXA) is a modality of improving the care of this patient group. We focused on the use of perioperative TXA and its benefits in hip fracture patients. This prospective study looked at the use and outcomes of TXA in hip fracture patients of St Vincent's University Hospital (SVUH) since February 2019.

Close to seventy patients received TXA perioperatively. Data on haemoglobin levels and American Society of Anaesthesiologists (ASA) scores were collected using patient-online laboratory results and MetaVision, respectively. Data on patients experiencing a deep venous thrombosis (DVT) and/or pulmonary embolism (PE) was collected using the radiology reports on Syngo.

These 65 patients had a mean ASA of 2.5. Their mean haemoglobin drop pre-op to day 3 post $\mathrm{Op}$ was $2.53 \mathrm{~g} / \mathrm{dl}$. The percentage patients who received intraoperative transfusion was $0.05 \%$. No patient suffered a DVT and 1 patient had a PE (rate of $0.03 \%$ ). This patient did not have any clinical symptoms and the PE was an incidental finding on an abdominal ultrasound. On the subsequent CTPA it was described as a "trace left sided pulmonary embolism". This was most likely a third order PE. It is known from existing literature of a similar cohort that were treated without tranexamic acid had a mean $\mathrm{Hb}$ drop of $3.5 \mathrm{~g} / \mathrm{dL}$. Overall this study indicates that the use of TXA does not increase the post-operative risk of PE or DVT. No other adverse events were noted with the use of tranexamic acid. This research is being continued currently but we currently cautiously recommend the continued use of Tranexamic acid in hip fracture patients.

\section{We Are Roma-The Outsiders! An Overview Of The Romani People In Ireland}

Sasha Selby ${ }^{1,2}$, Aoife O'Sullivan ${ }^{3}$, Claire Sharkey ${ }^{1}$, Katie Lynam ${ }^{1}$, Cillian Lineen ${ }^{1}$, Niall Byrne ${ }^{1,2}$, Peter O'Reilly ${ }^{1}$, Clodagh O'Gorman ${ }^{1,2}$, Anne-Marie Murphy ${ }^{1}$

Dept. of Paediatrics ${ }^{I}$, University Hospital Limerick; Graduate Entry Medical School (GEMS) ${ }^{2}$, University of Limerick; School of Medicine ${ }^{3}$, University College Dublin

\section{$\mathrm{MW} / \mathrm{UL}$}

Background and Aims: The Romani people colloquially known as the "Roma Gypsies" are an indo-Aryan ethnic minority group originating from the northern Indian subcontinent, traditionally itinerant and estimated to number between 4000 and 5000 in Ireland at any one time.

Often overlooked, nomadic races are unique minority groups with specific health needs

Disadvantaged minority groups are known to bear a greater burden of illness than general populations

Cultural competency and cultural awareness amongst healthcare professionals when dealing with the ethnic diversity in modern Irish Society is crucial.

This study aims to identify the specific family structure, common genetic conditions and other illnesses associated with the Roma Gypsy Subgroup within the context of the Irish Population.

Methods: A review of the current available literature and a database search were undertaken and relevant Clinicians and organisations contacted in an effort to compile data on the Irish Roma to inform appropriate action in the area of Roma Child Health. 
Results: Roma Gypsies are a distinct subgroup that are described across almost all bodies of literature as being discriminated against in both institutional and individual levels, some even describe this discrimination to be a "Roma Gypsy Holocaust".

At present there are no current Irish studies that describe the ethnic variety and cultural norms of this important subgroup in Irish society.

The most striking piece of international literature reported that currently up to $20 \%$ of Roma Gypsies described experiencing poverty that would be described to be "Extreme". In 50\% of households with children, it was reported that food was seldom available and that malnutrition was a massive cause of morbidity among Roma Children.

Conclusion: Our review should prompt formal Irish data collection on this nomadic race. Further studies are required to analyze the impact of marginalization on Irish Roma Gypsy Children in relation to their unique issues and needs.

\section{A Study Of Electroencephalography (EEG) Patterns In Autoimmune Encephalopathy}

Bowers L, McNamara B

Dept. of Neurophysiology, University Hospital Cork, Wilton, Co Cork

\section{$\mathrm{S} / \mathrm{UCC}$}

Introduction: Autoimmune encephalopathy (AIE) describes a group of disorders where auto-antibodies attack neurons and disrupt cell signalling within the brain.

Aims \& Objectives: The purpose of this study was to assess the utility of electroencephalography (EEG) in Autoimmune Encephalitis (AIE). Electroencephalography (EEG) and MRI brain findings were compared in subgroups differentiated by serology.

Methods: This was a retrospective descriptive case series which involved reviewing EEGs performed on patients diagnosed with AIE between November 2010 and December 2018. Patient data was reviewed in the Neurophysiology Department at Cork University Hospital; a large tertiary referral centre. Databases from the immunology and neurology departments provided sixteen patients. Patients met inclusion criteria once EEG, MRI, serology, cerebrospinal fluid analysis and consultant neurologist care was identified in medical records. Patients were excluded if infective, traumatic, structural, or alternative pathology was identified. One patient was identical on both databases, two patients did not receive serology and alternative diagnosis was made in one patient. Eleven patients were eligible for participation. All EEGs were reviewed for normal alpha activity, abnormal slow/theta/delta frequencies, symmetry, epileptiform/seizure activity and specific patterns (e.g. Extreme Delta Brush EDB). EEG, MRI and serology results were compared in all patients.

Results: Anti-NMDA antibodies and anti-LGI1 antibodies were detected in five and two patients respectively; the final four patients were seronegative. Abnormal EEG findings occurred in $91 \%$ of patients. Generalised slowing, asymmetrical slowing and epileptiform activity was detected in $81 \%, 63 \%$ and $36 \%$ respectively. Normal Alpha activity was detected in $72 \%$ of cases. EDB was detected in two patients with anti-NMDA antibodies. Abnormal MRI findings was found in two patients with anti-LGI1 antibodies and two seronegative patients. All patients with anti-NMDA AIE demonstrated EEG abnormalities with normal MRI brain results. Conclusion: EEG is a major contributor in the diagnosis of AIE. While abnormalities are non-specific they indicate organic illness and raise the suspicion of AIE. Every patient presenting with unexplained neuropsychiatric symptoms should receive EEG.

Capnocytophaga canimorsus- A Rare Case Of Overwhelming PostSplenectomy Infection And Sepsis From Dog Bites In The Irish Population

Liew S, Maraj RS, Abdul Ghafour SF
Dept. of Medicine, South Tipperary General Hospital, Clonmel, Co Tipperary, Ireland

\section{S/UCC}

Introduction: Capnocytophaga canimorsus is a Gram-negative bacillus commonly found in canine and feline oral flora. Infection by $C$ canimorsus is rare in the healthy population. There are only two other published cases of $C$ canimorsus-induced sepsis in the Irish population. Infections are mostly transmitted by dog bites. The most recognized risk factors for this infection are splenectomy, alcoholism, and immunosuppression from other causes. Clinical features may range from mild to fulminant. However, the majority of documented cases presented with overwhelming post-splenectomy infection (OPSI) culminating as sepsis, which has a mortality rate of up to $30 \%$ despite adequate treatment.

Case Presentation: AT is a 66-year-old woman with autosplenectomy secondary to systemic lupus erythematosus (SLE) who developed $C$ canimorsus-induced OPSI and sepsis contracted from dog bites. She presented with a two-day history of disorientation, frontal headache, empty retching, generalized weakness, lethargy, dizziness, dry mouth, and transient dysarthria lasting for half an hour. Her vitals and blood results were concerning for sepsis, and the sepsis protocol was commenced. Her hands showed puncture wounds, which she elucidated were dog bites sustained three-days prior. Clinical exam was otherwise unremarkable. Blood cultures grew Gram-negative bacillus that were identified as $C$ canimorsus on day 8 . Ultrasound abdomen noted asplenia. She was managed with Piperacillin-Tazobactam, Vancomycin, Gentamycin and Co-Amoxiclav. Upon making a full recovery, she was discharged with Phenoxymethylpenicillin and advised to have routine prophylactic vaccinations.

Discussion \& Conclusions: $C$ canimorsus is an emerging cause of OPSI and sepsis in various countries including Ireland. Clinicians should be vigilant of $C$ canimorsus infections in asplenic patients, immunocompromised patients, and alcohol abusers who present with signs and symptoms of an infection with a history of exposure to dogs or cats. They should be treated with intravenous antibiotics immediately, and subsequently covered with prophylactic measures to prevent future infections.

Atypical Presentation Of Metastatic Melanoma In A 21-Year Old Male- A Synchronous Klebsiella Infection

McConnell A, Goggin C, Donnellan P

Dept. of Oncology, University Hospital Galway

\section{WNW/NUIG}

Introduction: Melanoma is ranked as the $5^{\text {th }}$ most common malignancy in Ireland, causing 159 deaths in $2018^{1}$. Earlier recognition of this malignancy will reduce these numbers. An awareness of atypical presentations of melanoma is essential to achieve this.

Case: We describe the case of a 21-year old Australian male who presented with an acutely enlarging and painful right axillary mass, associated with fevers and a recent travel history to Asia. His clinical picture and blood markers were strongly suggestive of a septic reaction to an abscess or lymphadenitis. The biopsy sample of the lymph node mass cultured Klebsiella and he commenced antibiotics. Unexpectedly, histology returned days later diagnosing metastatic melanoma, positive for BRAF mutation. A primary lesion was not identified, following dermatology and ophthalmology reviews. Radiology discovered metastases to the lungs. The combined therapy of Dabrafenib and Trametinib produced an immediate response, measured by reduction in pain and size of the mass and an improvement in blood markers, which had not occurred with the antibiotic therapy.

Discussion: This case describes an atypical presentation of metastatic melanoma as it synchronously presented with an infection within the same lymph node. Research states that cancer cells can create an 
immune-suppressed environment to facilitate their growth ${ }^{2}$. Albeit the synchronous presentation was concluded to be an unlucky coincidence, this could have been a case of an opportunistic infection within an undiagnosed stage 4 immuno-compromised patient.

This patient had the BRAF mutation, as do $40-60 \%$ of patients with metastatic melanoma ${ }^{3}$. The COMBI-D and V trials, using a combination of Dabrafenib and Trametnib, demonstrated a significant response ${ }^{3}$, as seen in this case. Advantages of the dual treatment in comparison to the single agents are an increased duration of response and avoiding the cutaneous events, such as squamous cell carcinoma and skin eruptions ${ }^{4}$. References: National Cancer Registry Ireland. Cancer Factsheet Melanoma [online]. [updated May 2018; cited 13 Oct 2019]. Available from: https://www.ncri.ie/sites/ncri/files/factsheets/ Factsheet\%20melanoma.pdf [Accessed 13 Oct. 2019].

Alizadeh D, Larmonier N. Chemotherapeutic Targeting of Cancer-Induced Immunosuppressive Cells. Cancer Research. 2014;74(10):2663-2668.

Atkinson V. Recent advances in malignant melanoma. Internal Medicine Journal. 2017;47(10):1114-1121.

Martin R, Bachman M. Colonization, Infection, and the Accessory Genome of Klebsiella pneumoniae. Frontiers in Cellular and Infection Microbiology. 2018; 8.

\section{Anti-Hypertensive Audit}

Muhamad Fauzi S, O’MARA G

Dept??? Roscommon Hospital

\section{WNW/NUIG}

Introduction \& Aims: Uncontrolled hypertension is associated with increased risk of cardiovascular-related complications \& kidney disease. This audit aims to determine if the blood pressure of hypertensive patients were maintained within target range, and to see if the antihypertensive used are as per guideline.

Methods: An audit was carried out for one day on all patients admitted in Roscommon Hospital. Patients who had hypertension were included. A proforma looking at demographics, co-morbidities, average BP reading and medications were used for the audit. This audit referred ESC Arterial Hypertension 2018 Guidelines as standard.

Results: $57 \%$ of inpatients in Roscommon Hospital had hypertension. $67 \%$ of the antihypertensive patients were within target range. The remaining that were not in target; $23 \%$ were $10 \mathrm{mmHg}$ above target SBP, $7 \%$ were $20 \mathrm{mmHg}$ above while $3 \%$ were $30 \mathrm{mmHg}$ offtarget.

$60 \%$ were on 2 antihypertensives, $20 \%$ were on $1,14 \%$ were on 3 antihypertensives, $3 \%$ were on 4 antihypertensives and $3 \%$ were on none. ESC recommended medications tailored to their co-morbidities. Majority of patients with CAD, CKD, HF and stroke were on recommended antihypertensive combinations. However, only 55\% hypertensive patients with atrial fibrillation were on appropriate combinations \& $29 \%$ diabetic patients were on recommended antihypertensive.

Conclusions: More than half of the patients were hypertensive. $67 \%$ of these had their blood pressure controlled. $60 \%$ were on 2 antihypertensive, and most combinations were as per ESC recommendations, except for patients with atrial fibrillation and diabetes.

Discussions: Evidence has shown a decrease in $10 \mathrm{mmHg}$ SBP is associated with significant reductions in all major cardiovascular events by $20 \%$. However, as majority of patients in Roscommon Hospital are elderly, there is 'balancing' needed between the risk of orthostatic hypertension and the risk of increased cardiovascular complications.

Limitations: The BP readings of patients were determined by taking the most frequent reading. Most readings were stable; however a minority had erratic readings.
To Assess If NCCN Guidelines On Surveillance Of Uveal Melanoma Are Being Followed At Sligo University Hospital

Jordan $\mathrm{E}^{1,2}$, Patel $\mathrm{A}^{2}$, Yousif $\mathrm{A}^{1,2}$.

Dept. of Medicine ${ }^{1}$, National University of Ireland Galway; Oncology Dept. $^{2}$, Sligo University Hospital

\section{WNW/NUIG}

Introduction: Uveal melanoma is the most common primary ocular malignancy ${ }^{1}$. Research growing with potential for targeted therapy around reducing uveal melanoma tumorginesis ${ }^{2}$. The number of patients attending Sligo university hospital for surveillance of uveal melanoma has doubled since 2017. For these reasons it is pertinent that these patients are adequately followed up.

Aims \& Objectives: Aim to asses if $\mathrm{NCCN}$ guidelines $^{3}$ on surveillance of uveal melanoma are being followed.

Methodology: Type of study is quality improvement; first stage of audit with retrospective notes review on the surveillance of all uveal melanoma patients in Sligo University hospital.

Results:

$87 \%$ of patients are receiving CT contrast TAP 6 monthly for at least the first 2 years, $100 \%$ receiving some form CTTAP.

$75 \%$ - having clinic visit for LFTs alone without concurrent imaging $25 \%$ - received LFTs at time of imaging only

Genetic information and histology were documented and/or gathered in $12.5 \%$ of patients. Tumour size was documented clearly in $62.5 \%$ cases. If no risk stratification information, and, thus considering all patients high risk: $75 \%$ patients in Sligo currently meeting criteria for "high risk surveillance"

Conclusion: $75 \%$ of patients attending Sligo oncology had LFTs monitored more frequently than imaging. RCCN do not recommend LFTs alone without imaging as part of surveillance as poor sensitivity for early detection metastases. This should be reviewed as may be recording LFTs too often.

Risk factors should be gathered and documented more clearly in the chart, this could help individualise surveillance - information comes from Royal Victoria, Dublin. Better interdisciplinary communication recommended. If no risk stratification information: consider all patients high risk $75 \%$ patients in Sligo currently meeting criteria for "high risk surveillance". Ensure surveillance imaging +/-LFTs every 3-6 months for 5 years, then every 6-12 months for 10 years, then as clinically indicated if considering all patients "high risk".

\section{References:}

Virgili, G., Gatta, G., Ciccolallo, L., Capocaccia, R., Biggeri, A., Crocetti, E., Lutz, J.M., Paci, E. and EUROCARE Working Group, 2007. Incidence of uveal melanoma in Europe. Ophthalmology, 114(12), pp.2309-2315.

Yoo, J.H., Shi, D.S., Grossmann, A.H., Sorensen, L.K., Tong, Z., Mleynek, T.M., Rogers, A., Zhu, W., Richards, J.R., Winter, J.M. and Zhu, J., 2016. ARF6 is an actionable node that orchestrates oncogenic GNAQ signaling in uveal melanoma. Cancer cell, 29(6), pp.889-904.

NCCN Clinical Practice Guidelines in Oncology (NCCN Guidelines $\left.{ }^{\circledR}\right)$ "Uveal Melanoma”Version 1.2019 - June 14, 2019 NCCN.org

Investigation Of The Incidence Of Kidney Stones And Alternative Pathology On CT Kubs In Patients Presenting To The Emergency Department In University Hospital Galway - A Follow-Up Study

Coyle $\mathrm{M}^{1}$, O’Donnell $\mathrm{J}^{2}$, Waters $\mathrm{C}^{3}$, McCarthy $\mathrm{P}^{4}$. Depts. of Urology $y^{1}$, Emergency Medicine ${ }^{2}$, Nephrology $y^{3}$, Radiology ${ }^{4}$, University Hospital Galway 


\section{WNW/NUIG}

Introduction: Flank pain and lower abdominal pain have a broad differential diagnosis. Renal stones are a common pathology in the population and renal colic is a common presenting symptom to the ED. Careful history and examination are the keys to determining if a patient has renal colic and whether this necessitates CT KUB. A similar previous study in UHG in 2017 displayed that $51 \%$ of CT KUBs showed stones, $10 \%$ had alternative pathology and $80 \%$ of those with alternative pathology did not have stones.

Aims: To evaluate the incidence of renal stones picked up on CT KUBs and the presence of alternative pathology.

To compare these results to a similar study performed in UHG in 2017 and determine if there has been an improvement/disimprovement.

Methods: A retrospective study of 100 consecutive CT KUBs from the $13 / 10 / 19$ to the $25 / 11 / 19$ in the ED of UHG was performed and results compared to the study which took place in 2017. Analysis of the following was done; incidence of stones on CT KUB, incidence in males vs females, presence of alternative pathology on the CT, presence of clinically relevant alternative pathology.

Results:

$49 \%(n=49)$ of CT KUBs showed renal stones. Of those with stones, $59 \%$ $(n=29)$ were male and $41 \%(n=20)$ were female.

$65 \%(n=65)$ had alternative pathology seen on the CT.

$13 \%(n=13)$ had clinically significant alternative pathology, and of these, $62 \%(\mathrm{n}=8)$ did not have renal stones.

Conclusion: The findings of this study in UHG are similar to those in 2017. These results are consistent with other centres across the world (Rucker, Menias and Bhalla, 2004). This highlights the need for careful history and examination in order to select the most appropriate scan, and the importance of considering alternative diagnoses when faced with flank pain.

Reference:

Rucker, C., Menias, C. and Bhalla, S. (2004). Mimics of Renal Colic: Alternative Diagnoses at Unenhanced Helical CT. RadioGraphics, [online] 24(suppl_1), pp.S11-S28. Available at: https://pubs.rsna.org/doi/ full/10.1148/rg.24si045505 [Accessed 1 Dec. 2019].

\section{An Audit Of A Respiratory Integrated Care Programme In Primary Care}

Sheridan AL, O'Ciardha D, O'Callaghan M

GPs at Tallaght Cross, Dublin 24

\section{DSE/TCD}

Introduction: GPs at Tallaght Cross recently introduced a Respiratory Integrated Care (RIC) programme. This programme aims to improve the care of patients with chronic respiratory conditions, including asthma and Chronic Obstructive Pulmonary Disease (COPD). Through education, pharmacotherapy, lifestyle advice and pulmonary rehabilitation, the respiratory Clinical Nurse Specialist and physiotherapist work alongside GPs to help patients manage their chronic condition.

Aims: To assess the outcomes of the RIC programme by comparing the number of times patients present to their GP and require treatment with antibiotics and steroids.

Method: Data was collected for the 12-month period prior to their first consultation with the RIC team. The post-RIC data was collected from the date of first RIC appointment to a maximum of 15 months after the programme began. Data was collected from Socrates and analysed in Excel, including the number of GP attendances and courses of antibiotics or steroids prescribed.

Results:

44 patients were seen by the RIC team during the audit, including 21 with COPD, 10 with asthma and 13 with other respiratory problems. Post-RIC time ranged from 2 to 15 months. GP visits per year decreased by $6 \%(9.3$ pre-RIC and 8.7 post-RIC). Respiratory issues accounted for 6.2 visits
pre-RIC and 5.0 post-RIC, representing a $19 \%$ decrease. In the COPD/asthma subgroup antibiotic prescribing decreased from 2.15 courses per year pre-RIC to 2.03 post-RIC, steroid prescribing decreased from 1.75 courses per year to 1.49 .

Conclusion: Patients with COPD and asthma who were involved with the primary care RIC programme attended their GP less and required fewer courses of antibiotics and steroids than the previous year. Limitations include a shorter time frame post-RIC compared to one year pre-RIC. Data collected over a longer period of time will better assess the outcomes of the RIC programme.

\section{A Rare Comparison Of PCSK9 Inhibitor And Statin Regimes In A Case Of Refractory Familial Hypercholesterolaemia}

Johnson H, Conway M

Cardiology/General Medicine, St Luke's Hospital, Kilkenny

\section{DSE/TCD}

Background: Cholesterol plays a significant role in atheroma formation. It is widely documented that higher total serum cholesterol (TSC) leads to greater risk of developing atherosclerosis and consequently cardiac events such as premature myocardial infarction. Patients with very high levels of cholesterol $(\mathrm{TSC}>7.5 \mathrm{mmol} / \mathrm{L}$ ) due to Familial Hypercholesterolemia (FH) are at particular risk and often phenotypically present with cutaneous stigmata such as xanthelasmata and xanthomata. Aggressive cholesterol lowering is indicated in patients with and without $\mathrm{FH}$ in whom vascular cholesterol accumulation is proven. Whilst in the heterozygote cases where TSC is at or below the population average (c. $5.1 \mathrm{mmol} / \mathrm{L}$ ) statins work tolerably well, in patients with FH the resistance to cholesterol lowering is such that blockade of PCSK9 is now becoming an essential aspect of patient care.

Discussion: Here we describe a case of refractory FH in whom the TSC commenced at $14 \mathrm{mmol} / \mathrm{L}$ and was reduced to $2.7 \mathrm{mmol} / \mathrm{L}$ with dual PCSK9 inhibitor and statin therapy. Once TSC was successfully lowered to this level the statin was withdrawn at the patient's request; it was restarted after a subsequent rise in TSC. PCSK9 inhibitor treatment was then stopped due to withdrawal of HSE funding. The effects on TSC in response to each of these changes in medication is shown here. This unique set of circumstances offers a rare comparison between PCSK9 inhibitor monotherapy vs statin monotherapy vs dual therapy in a patient with refactory $\mathrm{FH}$.

Using Data From In-Built Software On NIV Devices And Overnight Oximetry Studies For Non-Invasive Ventilation Titration In Children

Campbell $\mathrm{M}^{1}$, O’Donohoe $\mathrm{PK}^{2}$, O’Reilly $\mathrm{R}^{2}$, Javadpour $\mathrm{S}^{2,3}$, Farrell $\mathrm{L}^{2}$, Cox DW ${ }^{2,4}$

${ }^{1}$ Mater Misericordiae University Hospital, Dublin/Mid Leinster intern training network (UCD)

${ }^{2}$ Respiratory Dept., Children's Health Ireland (CHI) Crumlin, Dublin.

${ }^{3}$ School of Medicine, Royal College of Surgeons in Ireland, Dublin, Ireland

${ }^{4}$ School of Medicine, University College Dublin (UCD), Dublin

\section{$\mathrm{DML} / \mathrm{UCD}$}

Introduction: Studies have shown that the apnoea hypopnea index (AHI) as measured by the in-built software on home ventilators correlates with AHI scores on polysomnography studies in children on non-invasive ventilation (NIV) ${ }^{1}$. Overnight oximetry $(\mathrm{OO})$ studies are a cheaper and a more readily available modality when compared with polysomnography. There have been no studies to date comparing the AHI from ventilator software with data from overnight oximetry studies. 
The aim of this study was to assess the correlation between AHI scores downloaded from CPAP devices and the oxygen desaturation index (ODI) on OO studies in children on nocturnal NIV.

Results: Matching data on AHI and ODI scores were available for analysis on 201 nights of overnight monitoring from 64 patients, with 47 nights of data available on 13 infants. The Pearson correlation coefficient between $\mathrm{AHI}$ and ODI scores was significant in both the infant group and the overall cohort measuring $0.689, \mathrm{p}=0.0001$ and $0.308, \mathrm{p}=0.0001$ respectively.

Conclusion: This data suggests that the AHI scores on in-built ventilator software correlates well with the ODI on overnight oximetry studies in children. This could prove a useful tool when titrating NIV pressures on children where PSG studies are hard to access.

Reference:

Khirani S, Delord V, Olmo Arroyo J, De Sanctis L, Frapin A, Amaddeo A et al. Can the analysis of built-in software of CPAP devices replace polygraphy in children?. Sleep Medicine. 2017;37:46-53.

\section{Emergency Urology Admissions, Admit To Purgatory And Hope!}

Flynn D, Hamed M, Mannion J, Bucholc M, Flanagan L, Sugrue M Surgical Dept., Letterkenny University Hospital

\section{WNW/NUIG}

Aims \& Objectives: Emergency surgery admissions account for over $11 \%$ of admissions globally ${ }^{3}$. Emergency admissions are associated with increased morbidity, mortality and pose the greatest challenge to our hospital service. Urology is the fourth busiest surgical discipline in Ireland ${ }^{4}$. Urology admissions account for a significant portion of Emergency admissions but their fate in model three hospitals is not clear. This study undertook an evaluation of the process, volume, outcome and length of stay. Of Emergency patients admitted to Letterkenny University Hospital. This study undertook an evaluation of the process, volume and outcome in terms of length of stay admitted to Letterkenny University Hospital.

Methods: A retrospective ethically approved audit was undertaken of patients admitted between January $1^{\text {st }} 2019$ and September 31st 2019 to Letterkenny University Hospital, data was obtained from the Hype coding system.

Results: 288 patients were admitted to Letterkenny University Hospital, mean age 45 (SD+/- 12 years). This accounted for $15 \%$ of the total of Emergency surgical admissions $(\mathrm{N}=1910)$. The median length of stay was 3 days (range 1 day to 18 days). The mean length of stay was 2.7 days (SD+/-2.9days). 12.2\% (35/288) were transferred to Galway University Hospital. 4/288 were accepted by the Urology service at Letterkenny University Hospital. 11\% (32/288) referred to OPD appointments at Letterkenny University Hospital. Patients were admitted to the following wards accordingly, Surgical 2 ward 92\%, Orthopaedics $4 \%$ and Paediatrics $4 \%$.

Conclusion: In conclusion Urology emergency admissions account for $12.2 \%$ of emergency surgery admissions to the hospital. And are held in a holding pattern without access to care. New revised protocols are urgently required to treat these patients.

\section{References:}

1.Department of Surgery, Letterkenny University Hosptial, Donegal Ireland

2.Emergency Surgery Outcome Advancement Project, Donegal, Clinical research Academy

3 Joaquim M. Havens, MD,y Pooja U. Neiman, MD, MPA, Braidie L. Campbell, MS,z Martin A. Croce, MD, $\S$ David A. Spain, MD, and Lena M. Napolitano, MDjj ,The Future of Emergency General Surgery, SURGICAL PERSPECTIVE Ann Surg 2019;270:221-222)

4. HSE RCSI Urology A model of care for Ireland Version 1: Summer 2019 Review: 2022 Model of Care for Urology CSP027/2019
Audit Of Compliance With Venous Thromboembolism (VTE) Prophylaxis Guidelines In Patients With Hospital Acquired VTE.

O’Rourke $\mathrm{E}^{1}$, O’Doherty $\mathrm{R}^{1}$, Tierney $\mathrm{A}^{1}$, Ewins $\mathrm{K}^{1}$, Buckley $\mathrm{R}^{2}$, Ní Ainle $\mathrm{F}^{1}$

Depts. of Haematology ${ }^{1}$, Quality \& Patient Safety ${ }^{2}$, Mater Misericordiae University Hospital, Dublin

\section{$\mathrm{DML} / \mathrm{UCD}$}

Background Hospital acquired thromboembolism (HAT) - defined as venous thromboembolism (VTE) occurring during or within 90 days following hospital admission - is a patient safety risk. In 2018 the HSE Acute Hospitals \& Quality Improvement divisions launched the first KPI estimating HAT. This pilot audit is the first to objectively validate and characterize VTE events occurring in MMUH arising from this KPI.

Aims: We aim to reduce the rate of HAT in MMUH by verifying the current rate and identifying root causes. In confirmed cases we determine whether formal VTE Risk Assessment (VTERA) was performed and whether appropriate prophylaxis was prescribed.

Methods: In the first quarter of 2019, 77 cases of HAT were identified of which we conducted a pilot review of 20 randomly selected charts. Radiology and chart review was completed.

Results: (N=20) 14 (70\%) had radiologically confirmed VTE during the defined period; 7 PEs (50\%), 5 DVTs (36\%), 1 Portal vein thrombus (7\%), 1 superficial thrombophlebitis (7\%). $12(86 \%)$ fulfilled criteria for HAT. All were provoked; provoking factors included IVDU (5), malignancy (3), surgery (2), recent admission (2). 0 patients had VTERA completed; thromboprophylaxis was indicated in 10/12 HAT cases $(83 \%)$, of which only 7 (70\%) had appropriate prophylaxis prescribed. $6(30 \%)$ had no radiologically confirmed new VTE; 5 had a history of VTE, 1 had DVT ruled out.

Conclusion: No cases of VTE had VTERA documented on admission; an essential step in determining individual thrombotic and bleeding risk factors. The implementation of sanctions for non-compliance with VTERA has potential to bring rates of appropriately prescribed thromboprophylaxis closer to $100 \% .25 \%$ (3/12) of confirmed HAT were potentially preventable with evidence of non-compliance with VTE prophylaxis guidelines. This demonstrates the urgency with which prevention strategies for HAT, in particular promoting VTERA, need to be prioritized to reduce iatrogenic morbidity and mortality.

Total Pelvic Exenteration: Complexity Of Decision Making And Quality Of Life Implications - A Case Report

Kneafsey $\mathrm{S}^{1}$, Kelly $\mathrm{ME}^{1}$, Mulsow $\mathrm{J}^{2}$

National Centre for Peritoneal Malignancy ${ }^{l}$, Dept. of Surgery ${ }^{2}$, Mater Misericordiae University Hospital, Eccles St, Dublin

\section{$\mathrm{DML} / \mathrm{UCD}$}

Background: Total pelvic exenteration is a potentially curative treatment for locally advanced primary or recurrent rectal cancer, but with considerable risk of morbidity and mortality.

Methods: We report an interesting and complex case of an advanced rectal cancer in an elderly patient with multiple medical issues. We outline the complexity of decision making and need for good peri-operative counselling.

Results: We report the case of a 75-year-old male referred to the National Peritoneal Malignancy Centre with peritoneal involvement from rectal carcinoma primary. He initially underwent chemoreduction therapy but showed very minimal response in his primary tumour, and was complicated by development of a colovesical fistula, myocardial infarction and three coronary stents. As part of his staging, his peritoneal carcinomatosis index (PCI) score was 5-6. 
He was counselled extensively regarding the high morbidity and mortality of the procedure, particularly considering recent medical issues and the complexity of the resection needed to clear all disease. A total pelvic exenteration was performed including an anterior resection, proctectocystectomy, prostatectomy, peritonectomy with adjacent nodes, ileal conduit, and end colostomy formation.

Discussion: This case outlines several important issues. Pelvic exenteration is an extensive procedure with a significant impact on quality of life. Associated morbidity and mortality is high, (30\% major morbidity risk, $2 \%$ mortality risk). This has substantial challenges regarding patient counselling and future quality of life implications. The use of decision aids can help shared decision-making and educating patients regarding the complexity of surgery.

Conclusion: Rectal cancer management has evolved substantially over the past two decades. The role of radical exenterative surgery can provide long-term cure. However this poses ongoing challenges with regards future morbidity and mortality, and impact on quality of life. Tailored individual treatment and use of decision aids help facilitate patient's input in their management.

\section{A Rare Aetiology Of Jugular Foramen Syndrome}

Feeney MM, Sabu CB

General Medicine, University Hospital Limerick

\section{$\mathrm{MW} / \mathrm{UL}$}

Introduction: Jugular foramen syndrome is characterized by dysfunction of the IX, X and XI cranial nerves. Causes of jugular foramen syndrome include a tumour at the skull base, nasopharyngeal carcinoma, trauma and infection.

Clinical Details: We report a case of an 80-year-old male who presented with two weeks of nasal regurgitation and swallow difficulty on a background of worsening hoarseness and dysphonia. His medical history included coronary heart disease with stenting, haemopericardium and atrial fibrillation. Clinical examination demonstrated a left deviating uvula, right deviating tongue and right sternocleidomastoid wasting, with no long tract signs or signs of cerebellar dysfunction or meningeal irritation. CT brain demonstrated an expanded, bulky right jugular foramen with bony erosion and MRI showed a nonhomogenous hyperintense region with irregular borders in the same location. The patient was transferred to a higher centre with expertise in skull base pathology and PET CT was suggested to investigate malignancy. A marked FDG avid lesion at the right skull base was identified, consistent with malignancy. Despite suggestion from the multidisciplinary team to commence radiotherapy, the decision was made to await biopsy results before starting treatment. CT guided biopsy demonstrated no malignancy which was confirmed on open biopsy. The biopsy demonstrated a chronic inflammatory process and cultures grew Pseudomonas aeruginosa, providing a final diagnosis of chronic osteomyelitis of the skull base secondary to Pseudomonas infection. The patient was treated with a long course of ciprofloxacin, became clinically well and repeat MRI after four month showed complete resolution.

Discussion: This case report outlines a rare cause of jugular foramen syndrome, chronic osteomyelitis, that presented like malignancy on scans. It demonstrates that chronic inflammatory processes should be considered alongside malignancy in the case of FDG avid lesions and emphasizes that a diagnosis of malignancy should be made by histopathology.

ICD Implantation As Part Of Myocarditis Management In Young Adult With Suspicion Of Underlying Genetic Cardiac Disease

Tio S W, Moore A, Kiernan TJ

Dept. of Cardiology, University Hospital Limerick
MW/UL

A previously healthy 22 year old gentleman was admitted following an out of hospital cardiac arrest. A total of 35 minutes of bystander CPR and DCCV x 4 was given due to ventricular fibrillation on ECG before return of spontaneous circulation was achieved. On admission, transthoracic echocardiogram showed severe left ventricular dysfunction (EF 15$20 \%$ ). He has normal coronary angiogram. A diagnosis of myocarditis was confirmed on cardiac MRI performed one week post arrest. After a month in the hospital, his heart function returned to normal and he recovered remarkably well following a post cardiac arrest hypoxic brain injury (HBI).

Current guidelines on ICD insertion in cases of myocarditis, a potentially reversible process, are equivocal. Details supporting ICD implantation in this case included a family history of sudden cardiac death and also potentially arrhythmogenic ECG changes observed during his admission. Factors against insertion of an ICD included the impact this would have on his life. With his background history of dyslexia, learning difficulty and poor performance in school, he finally discovered welding as a job that had turned his life around. Having ICD in-situ would mean he could no longer pursue this career. Furthermore, the patient was also recovering from HBI secondary to his cardiac arrest so obtaining valid consent was also of concern.

After many detailed discussions of the above with the patient and his family it was eventually decided to continue with ICD implantation. On his 6-week follow-up post discharge, he is doing well. He has been linked to neurologist and neurology occupational therapy for further rehabilitation post HBI and a genetic testing referral has been sent due to suspicious family history of a genetic cardiac disease.

This case serves as an interesting example of determining best patient care when faced with multiple medical and social uncertainties.

\section{A Cross-Sectional Audit Of Blood Pressure (BP) Control Among Medical Inpatients In A Tertiary Care University Hospital}

O'Sullivan C, Rothwell-Kelly G, O'Farrell-Tyler A, Holian J, O'Riordan A, Watson A, Waqas S

Dept. of Nephrology, St. Vincent's University Hospital, Elm Park, Dublin

\section{$\mathrm{DML} / \mathrm{UCD}$}

Aim: The national institute for health and care excellence (NICE) guidelines recommend a target BP of $<140 / 90$ ( $<80$ years old) and $<150 / 90$ (>80 years old) at all times amongst patients with hypertension. This audit gauged the adequacy of general medical inpatients' BP control and also assessed compliance with the recommended lifestyle interventions.

Methods: A prospective cross-sectional audit was undertaken and a total of 55 medical inpatients were selected by convenience sampling from various medical wards. Demographic information was recorded along with relevant medical history and medication record. The last two BP readings on patients' monitoring charts were averaged to assess control. Patients were also asked about lifestyle interventions.

Results: A total of 55 patients were included in the audit. Seventeen (30.9\%) were male. Ages ranged from 23-92. There were $50.9 \%$ $(n=28)$ with a diagnosis of hypertension. Of these $64.3 \% \quad(n=18)$ had adequate BP control. $82.1 \%(n=15)$ of the hypertension subgroup were on antihypertensive treatment $(71.4 \%$ on 1 medication, $50 \%>/=2$ medications). Among those with hypertension, $60.7 \%$ $(n=17)$ attempted a low salt diet and only $28.6 \%(n=8)$ undertook recommended exercise. Other lifestyle intervention results were varied, with no patients among those diagnosed with hypertension using mobile apps to record their BP. Amongst those with previously undiagnosed HTN $49.1 \%(n=27), 7.4 \%(n=2)$ had BP within the hypertensive range. 
Conclusion: In patients with a diagnosis of hypertension in the hospital, majority in this audit had BP level controlled as per the recommendations. Overall lifestyle intervention was poorly followed, although $72.7 \%$ of patients had been advised about, how it can affect BP. There is a need for educating these patients further about lifestyle intervention. There is also need for longitudinal monitoring of BP for the patients who were found to be hypertensive in this audit to establish definitive diagnosis amongst them.

\section{Renal Artery Stenosis Presenting As Stroke In A Gentleman With Few Cardiovascular Risk Factors}

Coughlan $\mathrm{AK}^{1,2}$, Loughlin $\mathrm{E}^{1}$, Casserly $\mathrm{L}^{2}$

Geriatric Dept. ${ }^{1}$; Nephrology Dept. ${ }^{2}$, University Hospital Limerick

\section{$\mathrm{MW} / \mathrm{UL}$}

We present the case of a 44 -year-old gentleman who presented with rightsided limb weakness, slurred speech and right-sided facial droop over 48hours. He had malignant hypertension of $250 / 165 \mathrm{mmHg}$. He had no past medical/surgical history, no regular medications, and had last attended his GP 3 years previously, when his blood pressure was 'high-normal'. He was a non-smoker, a mechanic and drank four units of alcohol daily. He had a negligible family history of ischaemic heart disease (mother and father, seventies).

Initial work-up revealed a creatinine of 200 , cardiomegaly on chest $\mathrm{x}$-ray and left ventricular hypertrophy on ECG. A CT brain showed small vessel disease and lacunar infarcts.

He was managed for a hypertensive emergency with end-organ damage. His blood pressure was treated with a calcium channel blocker, betablocker and alpha-blocker. Fundoscopy revealed grade-III hypertensive retinopathy. His secondary hypertensive screen was normal. His LDL was $4.3 \mathrm{mmol} / \mathrm{L}$. An echocardiogram confirmed moderate concentric left ventricular hypertrophy, EF $50 \%$. Cardiac monitoring was normal. CT aortogram out-ruled coarctation of the aorta. MRI brain showed chronic lacunar infarcts and microhaemorrhages, and acute cerebellar and lacunar infarctions. Renal ultrasound demonstrated symmetrical kidneys, $10.3 \mathrm{~cm}$. A renal MRA showed bilateral renal artery stenosis, with diameters of $3 \mathrm{~mm}$ proximally and $7 \mathrm{~mm}$ distally.

The patient was referred for renal artery stenting, performed on the right side only as the left showed only mild stenosis on angiography. This yielded positive results with an adequate blood pressure response.

The lack of risk factors for cardiovascular disease is remarkable in this case. The initial hypertension was difficult to control as ACEis were contraindicated. It was important to reduce the blood pressure slowly given it's chronicity. The management of acute stroke with anti-platelets was challenging in the presence of hypertension, micro-hemorrhages, and a pending surgical procedure.

\section{The Importance Of Genetic Counselling: A Case Of Recurrent Miscarriages}

Kamath P, Downey A, Woods K, Gannon M

Dept. of Obstetrics and Gynaecology, Midlands Regional Hospital, Mullingar

\section{DML/UCD}

This case is of a 31 -year-old woman, gravida 5 para $1+3$, who is currently pregnant. She has one living son and had 2 early miscarriages and 1 late miscarriage at $22+4$ weeks (dichorionic diamniotic twins). She has no other significant medical history.

Because her son has an intellectual disability, the recurrent miscarriages raised suspicion of a possible genetic correlation between her son's condition and the miscarriages. Foetal skin biopsies and placental samples were sent for genetic analysis from the miscarried twin foetuses and a post-mortem was conducted.

Post-mortem revealed chorioamnionitis as the cause of miscarriage and a heterozygous deletion on chromosome $\mathrm{X}$ in both mother and twins. Genetic analysis reported a likely pathogenic, non-polymorphic deletion containing 25 genes including SOX3 and ATP11C genes, former of which is linked to intellectual disability. However, it did not establish whether the deletion caused foetal death. The patient was counselled by a geneticist where she was informed of the possibility of the same deletion occurring in future pregnancies. Chorionic villus sampling was offered to detect the deletion in the current pregnancy, but was declined. She had reassurance that while her son and future pregnancies may carry the deletion, it probably wasn't the cause of her recurrent miscarriages.

This case presents an interesting scenario where a genetic cause for recurrent miscarriages wasn't identified, but genetic counselling still played an important role in the care of the patient and her family. It also highlights the significance of such counselling in singleton versus multiple pregnancies.

\section{Fahr To Go To Understand This Rare Disease}

O’Riordan A, Kiely L, Hannon M

Dept. ??Bantry General Hospital, Cork

\section{S/UCC}

Introduction: Fahr's disease is a rare neurodegenerative condition characterised by calcium deposition in the basal ganglia. It is inherited in an autosomal dominant fashion and symptoms usually occur between ages 40 and 70 .

Case: A 68-year-old man presented with a 3-day history of ataxia, confusion and slurred speech. A collateral history reported increasing disorientation over the previous 3 days and episodes of unsteadiness when walking. He was otherwise well with no focus of infection. He had no significant medical history. His examination was notable for ataxia and slurred speech but no other neurological deficits. His initial blood results were unremarkable. He had a CT Brain which showed dense calcifications in the basal ganglia and cerebellar hemispheres bilaterally. These radiological findings were consistent with Fahr's disease. Further investigations, including a lumbar puncture, were non-contributory. His symptoms improved during his admission without any major intervention. He will be monitored closely for progression of his disease in the coming years.

Discussion: Fahr's disease can manifest with a variety of neurological or psychiatric symptoms. Patients may first present with cognitive impairment, seizures, headache, dysarthria, parkinsonism or psychosis. The diagnosis of Fahr's disease is usually made by the characteristic CT findings of bilateral calcification of the basal ganglia along with progressive neurologic dysfunction. Several mutations have been identified as a possible cause of Fahr's disease, but no single gene has been identified. There is currently no specific treatment for Fahr's disease and management is primarily symptom-based. Prognosis is variable but eventually leads to progressive neurological decline. There is certainly a great deal more to be learned about this disease.

\section{References:}

Saleem, S., Aslam, H.M., Anwar, M. et al. Fahr's syndrome: literature review of current evidence. Orphanet J Rare Dis 8, 156 (2013) doi:10.1186/1750-1172-8-156

Malik, R., V. K. Pandya, and D. Naik. "Fahr disease-A rare neurodegenerative disorder." Indian Journal of Radiology and Imaging 14.4 (2004): 383.

\section{A Case Report Of Hyperparathyroidism In End Stage Renal Disease}

Byrne R, Watson A

Dept. Nephrology, St. Vincent's University Hospital, Dublin 


\section{$\mathrm{DML} / \mathrm{UCD}$}

Introduction: Hyperparathyroidism in end stage renal disease (ESRD) is common. It is usually secondary hyperparathyroidism and occasionally it can be tertiary. Tertiary hyperparathyroidism, due to longstanding secondary hyperparathyroidism, is characterized by nodular hyperplasia of the parathyroid glands due to autonomous parathyroid hormone secretion and is associated with hypercalcemia (Yuen, NK et al. 2016). Surgical treatment for secondary and tertiary hyperparathyroidism is removal of 3 and $1 / 2$ of the parathyroid glands, leaving half a gland in place for calcium homeostasis.

Case: We report a case of hyperparathyroidism with hypercalcemia in end stage renal disease. A 47-year-old female presented with features of hypercalcemia, despite recently starting on dialysis for autosomal polycystic kidney disease. On admission, her corrected calcium on admission was $3.72 \mathrm{mmol} / \mathrm{L}$ and PTH was $246 \mathrm{pg} / \mathrm{ml}$. This persisted following a previous resection of 3 parathyroid glands a few months prior. The pathology of the glands removed showed normal parathyroid tissue in all sections with no evidence of hyperplasia.

Extended Sestamibi showed a large single nodule in her anterior mediastinum which was confirmed to be a large anterior mediastinal mass on CT. This was removed surgically and histology of this gland confirmed a parathyroid adenoma. Following removal, she had resolution of her hyperparathyroidism and hypercalcemia.

Conclusion: Hyperparathyroidism in ESRD when accompanied by hypercalcemia indicates primary or tertiary hyperparathyroidism. Occasionally hyperparathyroidism in ESRD is due to a primary adenoma and not nodular hyperplasia. About $25 \%$ of ectopic parathyroid glands are found in the mediastinum (Lyden, Wang and Sosa, 2019). There is a role for CT and extended Sestamibi when hyperparathyroidism and hypercalcemia persist following parathyroid surgery.

References:

Lyden, M., Wang, T. and Sosa, J. (2019). Surgical anatomy of the parathyroid glands. [online] Uptodate.com. Available at: https:// www.uptodate.com/contents/surgical-anatomy-of-the-parathyroidglands\#H1 [Accessed 20 Nov. 2019].

Yuen NK, Ananthakrishnan S, Campbell MJ. Hyperparathyroidism of Renal Disease. Perm J. 2016;20(3):15-127. doi:10.7812/TPP/15-127

\section{Are Our Patients Receiving Adequate DVT Prophylaxis? A Clinical Audit}

Van de Hoef D, O'Mara G

Dept. of Medicine for the Elderly, Roscommon County Hospital, Roscommon

\section{WNW/NUIG}

Background: 9\% deaths annually are VTE-related, and for numerous factors, hospitalisation worsens our patients' risk. It is thought that $70 \%$ of all hospital-acquired VTE's are preventable with appropriate prophylaxis.

Standard: University College Hospital Galway's Kardex guidelines for DVT prophylaxis are the standard for prescribing. Primary choice for DVT prophylaxis locally is LMWH with the standard being Enoxaparin. Correct prescribing as per the guidelines are $40 \mathrm{mg}$ Enoxaparin OD unless their $\mathrm{CrCl}$ is $<30$, or weight is $<50 \mathrm{~kg}$, in which a reduced dose of 20mg Enoxaparin OD is recommended.

Target: The target would be for our institution to have $100 \%$ adherence to the prescribing guideline.

Methodology: On a single day, charts of all of the inpatients in Roscommon County Hospital were analysed for data pertaining to the following variables: Age, Gender, the prophylaxis prescribed, weight. $\mathrm{CrCl}$ was calculated using Cockroft-Gault formula including their most recent Creatinine found on our local laboratory reporting system.
Results of first cycle: In the total 49 patients, a prescribed dose error was seen in $24 \%$ of our patients. Of these errors, $37 \%$ were receiving too low of a dose, whereas $63 \%$ were receiving too high of a dose. Of those receiving too high a dose, $80 \%$ of these patients were $<50 \mathrm{~kg}$, the other $20 \%$ having a $\mathrm{CrCl}<30$.

Action plan: The plan is to complete a second audit cycle. The guidelines are present and available within the Kardex's and should be consulted in any uncertainty. Emphasize the importance of calculating Creatinine Clearances and having up to date weights.

\section{The Quality Of GP Referral Letters To A Dermatology Clinic; Poor} Quality Leads To Poor Triaging

Rynne R, Bourke J

Dept. of Dermatology, South Infirmary Victoria University Hospital, Cork

\section{S/UCC}

Introduction: Triaging is a process by which patients are prioritised into categories based on urgency. The accuracy of triaging is dependent on the information in the referral. Currently, there are no dermatology referral guidelines in Ireland or standardised means of assessing the quality of dermatology referrals. Studies demonstrate that referral quality is low, making triaging difficult. The exact relationship between referral quality and triaging remains unknown.

Aim: The aim of this study was to establish the relationship between the referral letter quality and the accuracy of triaging, and to determine other factors influencing triaging.

Method: Over a ten-month period, 90 GP letters of newly referred patients to the South Infirmary Victoria University Hospital's dermatology department were gathered. The necessity of referrals was assessed using NHS Scotland's Dermatology Referral Criteria and The National Clinical Programme for Dermatology's Exclusion Letter. The quality of referrals was measured using a novel referral quality assessment instrument, based primarily on the National Standardised Patient Referral Template (NSRT), with combined inputs from NHS Scotland's Dermatology Pathway and international studies.

Results: Overall, $36.7 \%$ of the referral letters were triaged incorrectly and $22 \%$ of referrals were shown to be unnecessary. The relationship between referral quality and accuracy of triaging was not statistically significant $(p<0.16)$. There was a significant correlation between use of the NSRT and referral quality $(\mathrm{p}<0.01)$. Letters containing the correct diagnosis were more likely to be triaged correctly $(\mathrm{p}<0.014)$. Other factors influencing triaging included having a clear reason for referral and the degree of concern expressed by the GP.

Conclusion: In Ireland, GPs are not guaranteed any formal dermatology training. This study demonstrates that GP education may be a beneficial tool in improving the accuracy of triaging. Furthermore, this study shows that templates improve referral quality and suggests that guidelines are required to reduce inappropriate referrals.

\section{A Review Of Discharge Prescriptions From Acute To Community Hospital Settings}

Mulroy S, Gaffney G, Mulligan L

Pharmacy Dept., Sligo University Hospital, the Mall, Rathquarter, Sligo

\section{$\mathrm{S} / \mathrm{UCC}$}

Background: National and international organisations have identified medication safety as a key priority; HIQA aims to reduce the rate of medication errors through its Medication Safety Monitoring Program, and the WHO aim to reduce avoidable medication errors by $50 \%$ in the next 5 years through its 2017 Global Patient Safety Challenge "Medication without harm". Medication errors commonly occur during 
transitions of care; at least $62 \%$ of patients have at least one unintentional error during transitions. ${ }^{1}$

Objectives: To identify and quantify medication errors on discharge prescriptions from acute to community hospital settings in a cohort of elderly patients discharged from Sligo University Hospital.

Methods: Data was collected on elderly patients $>65$ recently discharged from SUH to one of 3 community rehab hospitals between March-April 2019. Their discharge prescriptions were reviewed for medication errors, and the frequency and type of errors analysed.

Results: 85 errors were recorded. These included incorrect drug name/dose/frequency(39\%), medication incorrectly included(12\%), medication omission(12\%), incorrect anti-microbial(7\%), no indication for medication $(6 \%)$, monitoring incomplete $(6 \%)$, medication duplication $(4 \%)$, drug interactions $(4 \%)$, incorrect medication timing $(3 \%)$, and other errors $(7 \%)$.

Discussion: $39 \%$ of errors relate to incorrect drug name, frequency, or dose, meaning patients are either over- or under-dosed; particularly antithrombotics and direct oral anti-coagulants, where there is a fine balance between risk of ischaemic versus haemorrhagic events.

$18 \%$ of errors resulted in unnecessary polypharmacy, such as medications incorrectly included, or included without indication; most commonly analgesia and PPIs. Polypharmacy increases risk of drug interactions and adverse drug reactions, particularly in elderly populations.

$7 \%$ of errors relate to antibiotic use; particularly penicillin use without allergy status completed, and incorrect antibiotic choice.

Pharmacist medication reviews in community hospitals reduce the number of medication errors that reach patients, however this function is not always present

Audit Of Deep Vein Thrombosis (DVT) Pathway At Galway University Hospital (GUH): Interim Anticoagulation And Time From Referral To Diagnosis/Exclusion Of DVT In Emergency Department (ED)

Manshani V, Binchy J

Dept. of Emergency Medicine, Galway University Hospital

\section{WNW/NUIG}

Introduction: At GUH, patients with suspected DVT receive a full-leg venous duplex scan as diagnostic investigation which is only available Mon-Fri in vascular lab. If duplex scan is not organized on same day of ED presentation, an interim therapeutic dose of anticoagulation is offered to patients till their scan.

Aim: To audit compliance with:-

A) NICE guideline QS.29.2- Patients with suspected DVT have all diagnostic investigations completed within $24 \mathrm{hrs}$ of clinical suspicion. ${ }^{[1]}$

B) Patients with suspected DVT are offered an interim therapeutic dose of anticoagulation therapy if diagnostic investigations are not completed on same day of ED presentation.

Methods: Data was collected for all ED patients with suspected DVT who were referred to vascular lab for duplex scans between 01/07/201930/08/2019. Data includes time of assessment, time of referral and anticoagulation given/not given. Patients with suspected DVT already on oral anticoagulants were excluded from auditing compliance to standard B.

Results:

A) $70 \%$ (63/90) of patients had all diagnostic investigations completed with the duplex scan within $24 \mathrm{hrs}$ of clinical suspicion. 11 patients presented on Sat-Sun and 9.09\% (1/11) of them had duplex scans completed $<24$ hrs. 17 patients presented on Mondays and 70.59\% (12/17) of them had duplex scans completed $<24 \mathrm{hrs}$ but only $23.53 \%$ (4/17) were able to get scans on same day of presentation.

B) $79.6 \%(43 / 54)$ of patients received interim anticoagulation.
Conclusions: Majority of patients who presented over weekends had $>24 \mathrm{hrs}$ wait time and this seemed to have knock-on effect on patients presenting on Mondays who would have to wait for a next day scan and so on. To offload burden for appointments on Mondays, we plan on increasing reserved slots for ED on Mondays for duplex scans. Furthermore, we plan to update vascular lab booking forms to include a reminder for offering/providing anticoagulation and re-audit upon implementation of these plans.

\section{Reference:}

1) NICE. 2013. NICE Topic Expert Group and project team. [ONLINE] Available at: https://www.nice.org.uk/guidance/qs29. [Accessed 30 January 2020].

"Sometimes Two Wrongs Make A Right" - A Case Of Anti-GBM Disease With Persistently Negative Anti-GBM Titres

Rowan C' Sadlier D

Dept. of Nephrology, Mater Misericordiae Hospital, Dublin

\section{$\mathrm{DML} / \mathrm{UCD}$}

Introduction: Goodpasture's disease is a rare disorder characterised by a concomitant pulmonary-renal syndrome. It is often diagnosed by the detection of Anti-Glomerular Basement Membrane (AntiGBM) antibodies specific for $\alpha 3$-chain of IV-collagen resulting in catastrophic pulmonary haemorrhage and rapidly progressive glomerulonephritis (RPGN). Prompt diagnosis and appropriate management is key to survival in these patients. A $5 \%$ false negative rate has been ascribed for conventional ELISA ${ }^{1}$ thus complicating early intervention. We present one such case only rarely mentioned in literature ${ }^{2}$.

Case Presentation: Ms X, a 30-year-old smoker, was transferred to the Mater Hospital with a 5day history of haemoptysis with associated anaemia, haematuria, oligouria, bilateral flank-pain, dyspnoea and rigors. Her background included psoriasis, epilepsy and radiologically-isolated-MS. A GCS of 3 on presentation necessitated an ICU admission for intubation and ventilation. Initial investigations revealed creatinine 241, metabolic acidosis and bilateral pulmonary infiltrates on CT-Thorax. Her course was further complicated by frank haemoptysis requiring vigorous transfusions. Most notably, she had two negative anti-GBM titres with a third weakly positive. A renal biopsy confirmed active vasculitis. Her management in ICU included CVVHD, 13 cycles of plasmapheresis, 5 courses of pulsed methylprednisolone, 2 cycles of cyclophosphamide, 2 doses of rituximab, 4units RCC, 2 units fibrinogen, lunit platelets and a tranexamic acid infusion. Ms X recovered excellently and was discharged well to continue rehabilitation.

Conclusion: In conclusion, Anti-GBM disease accounts for a minority of RPGN. False negative ELISA warrant high clinical suspicion in patients presenting with RPGN and haemoptysis.

Entrapment of Anti-GBM Antibodies within renal/lung parenchyma result in serological levels below the limit of detection for ELISA and could explain the weakly positive result here ${ }^{2}$. Rare cases of IgA-IgM mediated anti-GBM disease, not detected by conventional ELISA, have also been described $^{3}$. If ELISA is negative, renal/pulmonary biopsies should be performed where clinical suspicion of Anti-GBM disease remains high. References:

Vries TB, Boerma S, Doornebal J, Dikkeschei B, Stegeman C, Veneman TF. Goodpasture's Syndrome with Negative Anti-glomerular Basement Membrane Antibodies. Eur J Case Rep Intern Med. 2017 Jul 13;4(8):000687. doi: 10.12890/2017_000687. PMID: 30755961; PMCID: PMC6346855.

Salama AD, Dougan T, Levy JB, Cook HT, Morgan SH, Naudeer S, Maidment G, George AJ, Evans D, Lightstone L, Pusey CD. Goodpasture's disease in the absence of circulating anti-glomerular basement membrane antibodies as detected by standard techniques. Am J Kidney Dis. 
Border WA, Baehler RW, Bhathena D, Glassock RJ. IgA antibasement membrane nephritis with pulmonary hemorrhage. Ann Intern Med. 1979 Jul;91(1):21-5.

\section{A Physically And Emotionally Costly Triad ... A Case Of Pseudo- Meigs' Syndrome}

Kelly S, O’Brien D

Dept. Gynaecology, St. Vincent's University Hospital, Dublin \& National Maternity Hospital, Dublin

\section{$\mathrm{DML} / \mathrm{UCD}$}

Introduction: Pseudo-Meigs' syndrome, a variant of Meigs' syndrome, is a rare clinical syndrome characterised by the triad of pleural effusion, ascites and a pelvic tumour, other than a benign ovarian fibroma, such as a leiomyoma of the uterus or secondary metastases to the ovary ${ }^{1}$. Its diagnosis is only truly made post-operatively, once the pelvic mass has been removed and resolution of the pleural effusion and the ascites as occurred. As its presentation mimics malignancy, it can pose diagnostic challenges for clinicians.

Case Presentation: A 49-year-old, para $2+0$ lady presented to the Emergency Department with a fever and a five-month history of right iliac fossa (RIF) discomfort and fullness. Her past gynaecological history was significant for dysfunctional uterine bleeding. On examination, her BMI was 35 and the abdomen was distended. Investigations revealed a haemoglobin of $8.4 \mathrm{~g} / \mathrm{dL}$ and CA-125 $179 \mathrm{U} / \mathrm{ml}$. Ultrasound abdomen pelvis revealed a right sided pleural effusion and ascites with a $22 \mathrm{~cm}$ heterogenous mass in the RIF, which was later biopsied. Conclusion at MDT was for abdominal hysterectomy with bilateral salpinogooopherectomy given the high likelihood of malignancy. The histology returned as a benign myometrial leiomyoma.

Discussion: Fibroids are extremely common and are clinically apparent in $25 \%$ of women of reproductive age ${ }^{2}$. A leiomyoma of the uterus is the most common cause of pseudo-Meigs' syndrome, as seen here. The elevated CA125 further increased suspicion for ovarian malignancy, thus necessitating surgery before the result of the biopsy returned. Although the surgical outcome would likely have been the same, patient distress could have been minimised. Conclusion: This case highlights the importance of not over-relying on non-specific tumour markers such as CA-125. Equally imaging techniques will not always help in giving the correct diagnosis. Benign tumours must always be in the differential of a pelvic mass, to limit patient anxiety and direct appropriate treatment.

References:

1. Zutshi V, Tiwari S, Sirswal S. Pseudo-Meigs' Syndrome Caused by Recurrent Leiomyoma Uterus. Indian Journal of Gynecologic Oncology. 2018;16(4).

2. Downes E, Sikirica V, Gilabert-Estelles J, Bolge S, Dodd S, Maroulis C et al. The burden of uterine fibroids in five European countries. European Journal of Obstetrics \& Gynecology and Reproductive Biology. 2010;152(1):96-102.

\section{A Case Of Abdominal Pain Post Subtotal Colectomy}

Phelan L, Martin S

Dept. of Colorectal Surgery, St Vincent's University Hospital

\section{$\mathrm{DML} / \mathrm{UCD}$}

Introduction: Ulcerative colitis is a chronic inflammatory bowel disease. If remission cannot be achieved or maintained through medical management, surgery may be required. This frequently involves a colectomy and ileostomy. Any major colorectal surgery is considered high risk for venous-thromboembolism. UC is associated with the highest 30-day VTE rate (2.74\%), followed by surgery for colorectal cancer (1.74\%) - American College of Surgeons: National Surgical Quality Improvement Program.
Case report: We present a 34-year old gentleman with an extensive medical history including UC, primary sclerosing cholangitis and cholangiocarcinoma, orthotopic liver transplant, and nephrotic syndrome. He developed a pancolitis that failed to respond to maximal medical management. His case was discussed at the MDM and then underwent a laparoscopic total colectomy and formation of end ileostomy. He was started on prophylactic enoxaparin post-op. He recovered well and was discharged 5-days later. He then represented to the ED with sudden onset abdominal pain. He was pale, sweaty, nauseous, and tachycardic. CT imaging showed an oedematous small bowel. Due to a high index of suspicion for VTE the images were re-reviewed on request and subsequently a partial superior mesenteric vein thrombus was diagnosed. He was treated with analgesia, LMWH, and bowel rest but failed to settle. He underwent a laparotomy which showed a grossly intact bowel, without evidence of ischaemia. Importantly there was no adverse effects on the liver transplant. He was treated with therapeutic enoxaparin and eventually discharged on a course of apixaban for 3 months.

Discussion: This case highlights the risk of VTE in colorectal surgeries and the increased risk with IBD, especially UC. It has prompted a focus on extended post-op chemoprophylaxis. As described by the Association of Coloproctology of Great Britain and Ireland consensus guidelines in surgery for IBD, as well as those for colorectal cancer patients, the recommendation now is for prophylactic enoxaparin or LMWH for a period of 28-days post op. This often requires patients to be taught how to administer the subcutaneous injections themselves before discharge.

\section{Empyema Sampling In Lung Decortication Surgery}

Phelan L, Healy DG

Dept. of Cardiothoracic Surgery, St Vincent's University Hospital, Dublin

\section{$\mathrm{DML} / \mathrm{UCD}$}

Empyema is a pathology of the pleural space in which purulent material accumulates in pleural space. It is often associated with pneumonia but can also be a ramification of lung carcinoma and immunosuppression status. Although radiological imaging is used to form a preliminary diagnosis, its true predictive value remains questionable.

Microbiological and histopathological records of 43 patients undergoing decortication were included in this review. Only patients who were diagnosed with late stages of empyema and underwent decortication were included in this study. The material obtained for the data was from broncho-alveolar lavage, pleural fluid, and decorticated material (pleu$\mathrm{ral} /$ lung tissue).

The broncho-alveolar lavage was performed with flexible bronchoscopy. Around $100 \mathrm{ml}$ of $0.9 \%$ saline water was instilled in a sub-segment of each lung and was sucked back. The lavage obtained was then stored and sent to lab in different containers under sterile conditions to prevent contamination. Microbiological examination, including gram stain and culture, was performed. The sample consisted of 43 patients -23 males and 20 females. For microbiology, $4.88 \%$ of BAL samples, $7.69 \%$ of tissue fluid samples, and $7.32 \%$ of pleural fluid samples were positive. For cytology/histopathology, $0.00 \%$ of BAL samples, and $5.41 \%$ of pleural fluid samples and $7.32 \%$ of tissue samples were positive. We recommend that, for the study and analysis of the microbiological samples, a myriad of all 3 different modalities of diagnosis is essential. However, tissue sampling is the preferred modality of diagnosis for cytology/histopathology owing to its ability to detect positive cases that cannot otherwise be detected.

\section{Blood Pressure Control In Acute Haemorrhagic Stroke In St James Hospital (SJH); An Audit Of Clinical Practice}

Browne C, Harbison J

Neurovascular Service, Medicine for the Elderly Dept. St James Hospital, Dublin 


\section{DSE/TCD}

Background: Acute blood pressure (BP) control is fundamental in the management of acute haemorrhagic stroke. In accordance with the AHA/ ASA 2015 guidelines, the SJH hyper-acute stroke protocol recommends a target systolic BP (SBP) $<140 \mathrm{mmHg}$. Based largely on the INTERACT-2 trial, the AHA/ASA guidelines recommend that lowering of SBP to $<140 \mathrm{mmHg}$ is safe and can be effective for improving functional outcomes.

Methods: A retrospective analysis of BP control in all patients admitted to SJH between 14/10/2018 - 14/10/2019 with acute haemorrhagic stroke was conducted via the Electronic Patient Record. SBP at first presentation, within 1-4, 24, 48 and 72 hours was recorded. Methods of acute BP control including parenteral (intravenous labetalol, GTN patch, labetalol infusion) and oral anti-hypertensive therapy within the first 72 hours were documented. The total number included was twenty-six patients $(n=26)$. Exclusion criteria $(n=13)$ included death within 24 hours, Beaumont Hospital transfer, miscoding.

Results: $90 \%$ of patients had a SBP $>140 \mathrm{mmHg}$ at presentation (mean SBP $171 \mathrm{mmHg}$ ). $62 \%$ remained hypertensive $>140 \mathrm{mmHg}$ at 4 hours. By 24 hours, $69 \%$ of patients $(\mathrm{n}=18)$ had SBP $<140 \mathrm{mmHg}$ (mean SBP $137 \mathrm{mmHg}$ ) with only $15 \%>160 \mathrm{mmHg}$ SBP. This rate was maintained at 48 and 72 hours. $57 \%$ of patients $(n=15)$ received at least one form of parenteral therapy. $90 \%(n=10)$ of those who received no parenteral BP treatment had SBP $<140 \mathrm{mmHg}$ at 24 hours versus $53 \%(n=8)$ of those who did receive parenteral therapy.

Discussion: Blood pressure within the first 24-72 hours of acute haemorrhagic stroke was well controlled in SJH. At least two-thirds of patients achieved an internationally standard target SBP $<140 \mathrm{mmHg}$. The most scope for improvement was within four hours of presentation and, interestingly, in the cohort that were treated with parenteral therapy. This suggests the need for more intensive escalation of blood pressure treatment in those requiring parenteral treatment.

References: Hemphill JC 3rd, Greenberg SM, Anderson CS, Becker K, Bendok BR, Cushman M, Fung GL, Goldstein JN, Macdonald RL, Mitchell PH, Scott PA, Selim MH, Woo D. Guidelines for the Management of Spontaneous Intracerebral Hemorrhage: A Guideline for Healthcare Professionals From the American Heart Association/ American Stroke Association. American Heart Association Stroke Council, Council on Cardiovascular and Stroke Nursing, and Council on Clinical Cardiology. Stroke. 2015 Jul;46(7):2032-60. Epub 2015 May 28.

Anderson CS, Heeley E, Huang Y, Wang J, Stapf C, Delcourt C, et al. INTERACT2 Investigators. Rapid blood pressure lowering in patients with acute intracerebral haemorrhage. N Engl J Med. 2013;368:23552365. doi:10,1056/NEJMoa1214609.

Audit Of Breastfeeding Rates And Artificial Formula Supplementation Of Breastfeeding Infants At UMHL

Haugh C, Shahrom N, Imcha, M

Dept. of Obstetrics \& Gynaecology, University Maternity Hospital Limerick

\section{MW/UL}

Background: The World Health Organisation recommends exclusively breast-feeding infants for the first 6 months of life. The Irish National Maternity Strategy recommends adherence to the WHO Code and BFI guidelines. Irish exclusive breast-feeding rates are $46.3 \%$. Supplementation with formula milk is found to decrease overall breastfeeding rates and duration.

Aims: Aims of this audit were to analyse exclusive breastfeeding rates in UMHL and formula supplementation of healthy breast-fed infants born to healthy mothers during their hospital admission.

Audit Design and Findings: Data was collected from patient records over a four-week period from two wards in September 2019. This was compared with the figures in the ward-book, where breast feeding statistics are obtained. Excel was used for data analysis. Key findings: $23 \%$ of all infants were exclusively breast fed, $38 \%$ combined, $37.5 \%$ were artificially fed and $0.5 \%$ not recorded. Comparing ward-book entries to those obtained from breastfeeding patients' notes found ward-book data showing $68.4 \%$ of breastfeeding infants as exclusive. The women's notes revealed only $36.4 \%$ of breast-feeders to be exclusive, identifying ward-book figures to overestimate exclusivity by almost double. Latch support was recorded for only $8.5 \%$ of first-time formula supplement entries. $75 \%$ of artificial formula supplements were given within the first 24 hours of life; with $23.4 \%$ given within the first 4 hours. Demographic factors and mode of delivery had little impact on supplementation rates.

Conclusions: Exclusive breastfeeding rates identified were markedly lower than the national average and the figures recorded in the wardbook. Combined-feeding rates were underestimated twofold. The commonest reason for supplementation was 'top-up' indicating a possible culture of supplementation at odds with WHO recommendations.

Recommendations: Improvements in record keeping and data collection; education for staff and mothers; increased breastfeeding support; and standardised protocols for every supplement given to breastfeeding mothers and reduced access to hospital provided formula.

\section{Efficacy Of Serial Intravitreal AVASTIN ${ }^{\circledR}$ Injection In Patients With Diabetic Macular Edema}

Lee JJ, Chee SY, Townley D

Dept. of Ophthalmology, University Hospital Galway

\section{WNW/NUIG}

Aim: To investigate the efficacy and safety of serial AVASTIN $®$ injection in the treatment of diabetic macular edema (DME).

Background: Poorly controlled diabetes promotes progression of diabetic maculopathy. Diabetic Maculopathy is the leading cause of blindness in the working population. It damages the macula which provides central vision. The mainstay of treating DME is with anti-vascular endothelial growth factor (anti-VEGF) injection to prevent macular retinal cysts and swelling (edema). AVASTIN® is use in ophthalmology as an off-label anti-VEGF intravitreal injection but the average duration of effect of AntiVEGF is 4 weeks. The seminal studies (DRCRnet, Read, Rise \& RIDE) show that the average number of injections required over the first year required 6-9 injections. The use of 6 injections on a monthly basis reduces the delay in real world practice in receiving and rebooking injections may help reduced overall macular edema.

Methods: This is a retrospective single-center study of patients with diabetic macular edema who had their $6^{\text {th }}$ injection of $1.25 \mathrm{mg}$ AVASTIN® intravitreal injection from April to July 2019. Clinical and OCT measurements were taken pre-injection and after 6 injections. All data were entered and analyzed in Excel.

Results: A total of 57 eyes receiving 6 AVASTIN® injections 4 weeks apart in 44 patients $(56 \%$ male) were included in the study. All of the indications were due to diabetic macular edema. All 3 OCT measurements showed significant improvement $(\mathrm{p}<0.05$ for all)

-Minimum retina thickness decreased from 311.55 to 277.61

-Average macular thickness decreased from 388.61 to 339.68

-Maximum retina thickness decreased from 505.88 to 447.35

Best-corrected visual acuity (LogMAR) at 0 and 6 months were 0.33 and 0.28 respectively $(\mathrm{p}=0.15)$. There was no case of endolphathalmitis, retinal detachment or significant changes in intraocular pressure after 6 months.

Conclusion: The use of serial AVASTIN $®$ injection in patients with macular edema secondary to diabetic is effective in maintaining good vision and improving clinical macular oedema after 6 injections monthly. It prevents delays in real world in rebooking patients for repeat injections 
during this time. The safety profile of AVASTIN $®$ is also consistent with previous studies.

\section{"Then Focus": An Audit Of Patients With Urosepsis In OLOL"}

Diong S, McNeill A, McKenna C, Talento AF

Microbiology and Pharmacy Depts., Our Lady of Lourdes Hospital, Drogheda

\section{$\mathrm{DNE} / \mathrm{RCSI}$}

The aim of this prospective audit was to investigate the "then focus" part of the RCPI "Start Smart then Focus" antibiotic care bundle. Twenty adult medical and surgical inpatients diagnosed with urosepsis or systemic urinary tract infection (UTI) on intravenous (IV) antibiotics were identified over 4 weeks. Patients were identified by communicating with postcall teams daily and consulting the Microbiology Laboratory log book for patients with gram negative bacteria in blood culture. Data was collected from patients' case notes and laboratory results in particular, blood and urine cultures and radiology.

Compliance with empiric treatment guidelines on the choice of agent(s) for urosepsis was $85 \%$., Gentamicin was discontinued by day 3 of treatment for $71.4 \%$ of patients. Areas for improvement identified include adherence to local guidelines on obtaining blood and urine culture prior to initiation of antimicrobial therapy and IV to oral switch. Only $57 \%$ of blood cultures and $45 \%$ of urine cultures were taken before antimicrobial therapy was started. A further $15 \%$ did not have their urine cultured at all. $75 \%$ of patients met hospital IV to oral switch criteria on day 3 of treatment however, only $27 \%$ were appropriately de-escalated. A possible reason for this is that only $41.2 \%$ of urine cultures were reported by day 3. As a result, more directed antimicrobial therapy could not be instituted and possible de-escalation not be carried out efficiently.

Longer IV treatments can lead to more hospital-acquired complications and can have socio-economic consequences for the patient and the state. Prolonged hospital stays can increase morbidity and mortality of elderly patients, and the likelihood of a complex discharge. Audit recommendations include adhering to guidelines on taking cultures before antibiotics are started where possible, and actively using the 'review' date section on the drug kardex as a prompt for IV to oral switch.

\section{Retrospective Evaluation Of Head Shape Triage Using The Three Screening Question \& Photo Approach}

McGurk C, Enright F

Dept. of Paediatrics, University College Cork, Cork

\section{$\mathrm{S} / \mathrm{UCC}$}

Introduction: Craniosynostosis (CS) is a rare congenital cranial malformation in which one or more cranial sutures have fused in utero leading to unfavourable neurological outcomes. The clinical presentation is similar to that of positional plagiocephaly, a more common and benign condition. Most paediatric head-shape referrals in Cork come from the community to the hospital. Following review, the babies with suspected CS are sent to Dublin for review by the cranio-facial team. Therefore, these babies have progressed through 3 different clinic attendances, causing expense, inconvenience and a potential delay in treatment. To address the inefficiency between community $\&$ hospital assessment, a new triage system was implemented. This involved the use of 3 screening questions previously studied in Denmark and photographs assessed by the Paediatrician and Craniofacial Surgeons.

Aim: To examine the effectiveness of 3 screening questions and photo approach to triaging head shape referrals and eliminate the need for local CT-scanning.

Methods: This study involved a retrospective cross-sectional chart review which was undertaken in Mercy University Hospital of 20 paediatric patients (median age 9 months, Sex 13 males, 7 females) who had been referred for head shape abnormality.

Results: The questionnaire triaged 10 of the 20 referrals as CS and 10 as positional plagiocephaly. Subsequent review of the photos of the patients gave a final diagnosis of 20 patients having positional plagiocephaly and 0 being diagnosed with $\mathrm{CS}$. Therefore, the photos were able to accurately diagnose $100 \%$ of the patients. CT scans decreased by $73 \%$ during this 12-month period.

Conclusion: The previous study in Denmark found the questionnaire to be a highly sensitive and therefore safe method for detecting CS but there were no cases of CS to test this conclusion. However, the questionnaire has low specificity due to high number of false-positives that were overcome by the use of the photographs. Therefore, it was felt that the photographs should remain the mainstay of the triage system as requested by the Craniofacial team in Dublin.

Lung Volume Reduction Surgery In Alpha 1 Antitrypsin Disease: A Case Series

Ni Fhlatharta, $\mathrm{M}^{1}$, Olaniyi, $\mathrm{J}^{2}$, Crotty, $\mathrm{T}^{1}$, McElvaney, $\mathrm{G}^{3}$, Eaton, $\mathrm{D}^{2}$ Dept. of Thoracic Surgery ${ }^{I}$, Mater Misercordiae University Hospital, Dublin; Trinity College ${ }^{2}$, Dublin; Dept of Respiratory Medicine ${ }^{3}$, Beaumont Hospital, Dublin

$\mathrm{DML} / \mathrm{UCD}$

Alpha 1 antitrypsin disease (AATD) is a genetic codominant condition and commonly causes severe lower lobe emphysema ${ }^{1}$.

AATD is the 4th most common indication for lung transplantation [2], however less invasive procedures like video assisted thoracoscopic (VAT) lung volume reduction surgery (LVRS) are alternatives to transplant, especially since the National Emphysema Treatment trial.

Due to the rare nature of this condition, few case reports exist on the Irish cohort. Therefore, we conducted a retrospective case review on three patients between 2016-2019 who underwent VAT LVRS at centres of excellence for thoracic surgery in Ireland. This paper reports on 3 patients with AATD who underwent LVRS, discussing preoperative and postoperative parameters such as pulmonary function tests and CT imaging. Improvements in post-operative PFTs were demonstrated in all patients. AATD induced COPD is a complex condition but LVRS is emerging as an additional option to medical therapy and transplantation for patients with end-stage emphysema. The above case series provides additional reporting of LVRS for a rare case of lung disease postoperative results were in keeping with NETT's conclusion that LVRS improves exercise capacity versus medical treatment alone for those with end stage emphysema.

References:

1. Choate R, Mannino D, Holm K, Sandhaus R. Comparing Patients with ZZ Versus SZ Alpha-1 Antitrypsin Deficiency: Findings from AlphaNet's Disease Management Program. Chronic Obstructive Pulmonary Diseases: Journal of the COPD Foundation. 2019;6(1):29-39.

2. McElvaney N. Diagnosing $\alpha 1$-antitrypsin deficiency: how to improve the current algorithm. European Respiratory Review. 2015;24(135):52-57.

\section{Fixing A Fractured System}

A.McCarthy ${ }^{1}$, L. Muller ${ }^{23}$, P. Keeling ${ }^{12}$, K. O’Shea ${ }^{12}$,

${ }^{1}$ Dept. of Orthopaedic Surgery, St Vincent University Hospital, Dublin, Ireland; ${ }^{2}$ Graduate Entry Medicine, University College Dublin; ${ }^{3}$ Intern Network: West-Northwest

\section{$\mathrm{DML} / \mathrm{UCD}$}

Introduction: The British Orthopaedic Association Standards for Trauma and Orthopaedics (BOAST) have produced guidelines advising 
that all patients be reviewed by an orthopaedic consultant within 72 hours of presentation. However, data from traditional fracture clinics rarely fulfil this criterion. Furthermore, data from the National Health Service (NHS) has determined that traditional fracture clinics have become unfit for purpose with low patient satisfaction rates, excessive waiting times and over 6.9 million missed appointments every year. Patients with nontreatment intensive fractures can be managed by a Clinical Nurse Specialist and appropriate follow-up physiotherapy, without needing the intensive consultant follow-up which a traditional fracture clinic would automatically require.

Objectives: To test the feasibility of a pilot virtual fracture clinic (VFC) with a view to reducing service costs and improving adherence to BOAST guidelines specified timeline for orthopaedic consultant review. Methods: A retrospective analysis of 103 patients referred to our pilot VFC from January $1^{\text {st }}$ to $31^{\text {st }} 2019$ was carried out. Injuries treated through the VFC included simple distal radius +/- ulnar fractures, fifth metacarpal fractures, shoulder dislocations, head/neck of humerus fractures, lateral malleolus fractures, AC Joint injuries, radial head/neck fractures, fourth metacarpal fractures, clavicle fractures, tibial plateau fractures, and patellar fractures. The primary outcome measured time from presentation to review by an orthopaedic consultant. A cost analysis was also performed to estimate the overheads and potential savings associated with VFC introduction.

Results: Following VFC establishment, time from ED presentation to review by orthopaedic consultant reduced from a mean of 229 hours to 72 hours $(\mathrm{P}=0.0001)$. Cost analysis demonstrated that the VFC created savings of $€ 3170$ per week, amounting to projected savings of $€ 38,040$.

Conclusions: VFC has the potential to improve clinical performance while delivering substantial financial savings.

References:

BOA Standards for Trauma (BOASTs). BOAST 7: Fracture Clinic Services, August 2013. British Orthopaedic Association. http://www. boa.ac.uk/publications/boast-7-fracture-clinic-services [Cited Dec. 2019].

Logishetty, K. (2017). Adopting and sustaining a Virtual Fracture Clinic model in the District Hospital setting - a quality improvement approach. BMJ Quality Improvement Reports, 6(1), pp.u220211.w7861.

An Audit Evaluating Current VTE Prophylaxis Performance In Tertiary And Secondary Level Hospitals (University Hospital Limerick And Nenagh General Hospital)

Talbot, $\mathrm{A}^{1}$, Tan, LY ${ }^{1}$, Tyrrell, $\mathrm{R}^{1}$, Ghanem, $\mathrm{Y}^{1}$, Mustafa, $\mathrm{M}^{1}$, Hamad, $\mathrm{I}^{2}$, Safaraz, $\mathrm{S}^{2}$, El Kholy, $\mathrm{K}^{1}$, Hoey, $\mathrm{M}^{1}$, O'Keeffe, $\mathrm{D}^{1}$, Watts, $\mathrm{M}^{1}$ Depts. of Medicine, University Hospital Limerick ${ }^{1}$, Dooradoyle, Co. Limerick; Nenagh General Hospital ${ }^{2}$, Nenagh, Co. Tipperary

\section{MW/UL}

Introduction: Venous thromboembolism (VTE) is a common complication of hospital admission. The VTE mortality rate in Ireland is approximately 4,000 per year, $48 \%$ of which are deemed preventable [1][2]. In 2008, University Hospital Limerick (UHL) conducted an audit showing inadequate thromboprophylaxis among medical patients. A medical proforma was subsequently introduced with a dedicated thromboprophylaxis prompt and follow up audits in 2012, 2015, 2018. This increased prophylaxis rates by $39 \%$ in high-risk patients by 2015 . This study aims to assess current practise of thromboprophylaxis among acutely ill hospitalized patients.

Methods: A cross-sectional study was conducted in UHL and Nenagh hospital (NGH) from September to November 2019. Exclusion criteria included patients on warfarin or therapeutic anticoagulation or those with anticoagulation contraindications. The overall risk was calculated using the Padua Score.[3] Anticoagulation prescriptions from medication lists were then recorded. Results: Two-hundred and forty-three patients (193 UHL, 50 NGH) were identified. One-hundred and eight-two were included following implementation of exclusion criteria. In UHL, the medical proforma was used in all patients. Seventy-three $(50.7 \%)$ thromboprophylaxis prompts were completed compared with $25 \%$ in 2018 . Twenty-one patients were low risk, forty-seven moderate risk and seventy-six were high risk. Of low-moderate risk patients and high-risk patients $60.3 \%$ and $76 \%$ received thromboprophylaxis respectively. This was a statistically significant increase in the high-risk group from $63 \%$ in 2018 ( $\mathrm{p}=0.071$ ). Overall thromboprophylaxis rates were significantly higher than in $2008(10 \%$ in low-moderate risk, $24 \%$ in high risk). Thromboprophylaxis rates in NGH were $76 \%$ in low-moderate risk and $80 \%$ in high risk patients.

Conclusions: VTE is a preventable life-threatening condition. Completion of the thromboprophylaxis proforma increased thromboprophylaxis rates in high risk patients. Despite being in the upper quartile of national figures (range 29.7\%-92\%), our thromboprophylaxis rates are still suboptimal.[1] Optimisation of VTE prevention strategies is vital to reduce patient exposure to potentially devastating outcomes.

References:

Health Service Executive (2018). Preventing Blood Clots in Hospitals. Improvement Collaborative Report, National Recommendations and Improvement Toolkit - HSE Quality Improvement Division

Thrombosis Ireland (2017) Irish Statistics accessed 26/11/18 http:// thrombosisireland.ie/wp-content/uploads/2017/10/irish-stats.pdf

Barbar S, Noventa F, Rossetto V, et. al. A risk assessment model for the identification of hospitalized medical patients at risk for venous thromboembolism: the Padua Prediction Score. J Thromb Haemost. 2010 Nov;8(11):2450-7.

The Use Of Heart Rate Variability Guided Exercise During Chemotherapy In A Triathlete With Triple Negative Breast Cancer

Talbot $\mathrm{A}^{1}$, McCabe $\mathrm{M}^{2}$, Daly $\mathrm{B}^{3}$, Gallagher $\mathrm{DJ}^{4}$

Dept. of Medicine ${ }^{1}$, University Hospital Limerick; ${ }^{2}$ Sports Med Ireland ${ }^{2}$, 32 Kildare St, Dublin; Janssen-Cilag Ltd ${ }^{3}$, Johnson \& Johnson, Airton Road, Tallaght, Dublin; Cancer Genetics Dept ${ }^{4}$., James' Hospital, James' Street, Dublin

\section{$\mathrm{MW} / \mathrm{UL}$}

Background: Heart rate variability (HRV) as a measure of autonomic activity has been used guide daily training in athletes. [1] This can be measured by the root mean square of successive differences (RMSSD) which measures the difference in time length between heartbeats conducted from the sinoatrial node. There are no current guidelines in Ireland for exercise during chemotherapy. We herein present the use of HRV-guided exercise during chemotherapy in a forty-four-year-old triathlete with breast cancer.

Case: A forty-four-year-old nulliparous triathlete was diagnosed with stage 1 BRCA1 mutated triple negative breast cancer. Investigations included a wide local excision which revealed a T1cN0M0 13.5mm grade 3 triple negative invasive ductal carcinoma. Surgical management involved bilateral mastectomy and oophrectomy. Adjuvant chemotherapy included four cycles of doxorubicin/cyclophosphamide and ten cycles of paclitaxel. The patient reported no side effects during chemotherapy with one episode of neutropenia. (absolute neutrophil count $0.7 \times 10^{9} / \mathrm{L}$ ). HRV was recorded by patient each morning to guide exercise for a two-year period. Six hundred and ninety HRV recordings were reviewed on the Training Peaks application. This application considers baseline HRV approximately $50-90 \mathrm{~ms}$. The lowest daily HRV recorded was $63.5 \mathrm{~ms}$ on cycle two day nine of AC chemotherapy from baseline of $80-100 \mathrm{~ms}$. HRV ranged from $63.5 \mathrm{~ms}$ to $101.8 \mathrm{~ms}$ during chemotherapy. Intense exercise was maintained throughout treatment guided by daily HRV without adverse events. This included strength and conditioning, cycling and running. This patient returned to international competitive sport within one year of treatment. This included acceptance into an international iron man competition involving a $3.86 \mathrm{~km}$ swim, $180.25 \mathrm{~km}$ cycle and $42.2 \mathrm{~km}$ run.

Conclusion: The case demonstrates the safe use of HRV-guided exercise during chemotherapy resulting in maintenance of fitness level and minimal 
chemotherapy side effects. HRV may be a useful adjunct for the individualisation of exercise programmes in the management of Irish cancer patients.

References: 1. Task Force Report. (1996) Heart rate variability: standards of measurement, physiological interpretation, and clinical use. Circulation 93:1043-65. doi: 10.1161/01.CIR.93.5.1043 Shaffer F, Ginsberg JP. (2017). An Overview of Heart Rate Variability Metrics and Norms. 5 :258. doi:10.3389/fpubh.2017.00258

How Does Limerick Breast Cancer Services Compares? Audit Looking At The Recurrence Of Breast Cancer In Patients Who Had Neoadjuvant Chemotherapy Between 2015-2016 At University Hospital Limerick, UHL

Fraser C, Aucharaz N, Devine M, Bradbury M, Tormey S, Merrigan A Breast Surgery Dept., University Hospital Limerick

\section{$\mathrm{MW} / \mathrm{UL}$}

Background: Neoadjuvant chemotherapy (NACT) was initially designed as a means of downsizing inoperable breast tumours. NACT allows for breast conserving treatment. NACT has been compared to adjuvant chemotherapy with results showing no significant difference. Met analysis done on the use of NACT in breast conserving therapy found locoregional recurrence to be $10.3 \%$ with overall survival rates being $75 \%$ at 8 years. Studies have highlighted that rate of recurrence is impacted by; pathological response, age of patient, initial size of tumor, clinical and pathological status.

Aims: Our goal is to compare the rate of locoregional recurrence of breast cancer patients who had NACT.

Methods: All data was collected from an oncology Share Drive. Our inclusion criteria consisted of all patients who had neoadjuvant chemotherapy followed by surgical intervention between 2015-2016. Out of 42 patients who met our criteria data was collected for 33. Currently still in the process of data collection.

Results: Pathological response was seen in all patients who had NACT, with $71 \%$ completed NACT while $21 \%$ of patients stopped treatment due to adverse sides effects. The rate of recurrence among patients who had NACT was found to be $0 \%$ in the group studied. This data shows NACT followed by surgical intervention may offer suitable patients up to a 4-year disease free period. Conclusion: NACT is used as the standard of care in management of locally advanced breast cancer in the University Hospital Limerick. Recurrence rates were found to be $0 \%$ 4years post intervention in this group of patients. Longer follow-up is needed to evaluate and compare UHL outcomes. By January 2020, we plan to accumulate more data from the period 2014-2016.

\section{Perforated Meckel's Diverticulum: A Case Report}

Alameer A, Sarwar M, Kabir U, Al-Khattab M, Butt J

Dept. of Surgery, Letterkenny University Hospital, Letterkenny, Co. Donegal

\section{WNW/NUIG}

Introduction: Meckel's diverticulum is known to be the most common congenital anomaly of the gastrointestinal tract ${ }^{1}$. The incidence of Meckel's diverticulum in the general population is close to $2 \%$. It results from the incomplete obliteration of the omphalomesenteric duct ${ }^{3}$. Major complications include bleeding, obstruction, inflammation, perforation and rarely malignancy. A wide variety of diagnostic tests exist for evaluation of suspected Meckel's diverticulum. However, pre-operative diagnosis remains a challenge.

Case Description: A 15-year old male presented to the emergency department at our hospital with a 3 day history of right iliac fossa pain, fever, nausea and vomiting. On examination the patient had tenderness in both iliac fossae, guarding and rebound. The patient had presented twice before with similar symptoms albeit less severe. On the first occasion a CT scan of the abdomen showed terminal ileitis and a microabscess in the right lower quadrant which was managed conservatively. On the second time a CT abdomen showed resolution of the aforementioned abscess and no abnormality otherwise. On this occasion, CT scan of abdomen and pelvis showed chronic inflammatory changes in the terminal ileum with acute appendicitis. Patient was taken to the operating room for a laparoscopic appendicectomy. A perforated Meckel's diverticulum was discovered intra-operatively and was managed with a diverticulectomy in addition to the planned appendicectomy. Post-operative course was uneventful and the patient was discharged home 3 days following the procedure. Conclusion: Meckel's diverticulum remains an important differential in cases of acute abdomen. Subacute presentations are possible and involvement of other disciplines such as paediatrics is essential for the optimum management of such cases.

References: Sagar J, Kumar V, Shah DK. Meckel's diverticulum: a systematic review [published correction appears in J R Soc Med. 2007 Feb;100(2):69]. J R Soc Med. 2006; 99(10):501-505. doi:10.1258/ jrsm.99.10.501

Bouhout T, Serji B, Egyir EU, et al. An unusual complication of Meckel's diverticulum: Littre's hernia. Pan Afr Med J. 2018;31:243. Published 2018 Dec 21. doi:10.11604/pamj.2018.31.243.10740

Robert Wyllie, Jeffrey S. Hyams, Pediatric Gastrointestinal and Liver Disease (Fourth Edition),W.B. Saunders,2011,Page iv, ISBN 9781437707748, https://doi.org/10.1016/B978-1-4377-0774-8.10095-8.

Broken-Hearted And Breathless: A Case Of Takotsubo Cardiomyopathy Secondary To Acute Asthma

Kennedy K, McEnery T, Akasheh N

Dept. of General Internal/Respiratory Medicine, St. James's Hospital, Dublin

\section{DSE/TCD}

Introduction: Takotsubo Cardiomyopathy (TCM) is an acute reversible cardiomyopathy induced by physical or emotional stress, most prevalent in postmenopausal women ${ }^{1}$. 'Takotsubo' means 'octopus pot' in Japanese and refers to the characteristic morphology of the left ventricular apex on echocardiography. ${ }^{2}$ Asthma is a rare driver of TCM with few previous reports.

Case Presentation: This case describes a 74-year-old woman admitted with an acute asthma exacerbation caused by respiratory syncytial virus bronchiolitis, who developed hypoxaemic respiratory failure secondary to acute pulmonary oedema. She had a background of non-ischaemic dilated cardiomyopathy, ejection fraction (EF) $40 \%$ and no inducible ischaemia on cardiac MRI. She presented with acute dyspnoea and diffuse wheeze on auscultation. Initially she responded to steroids and bronchodilators, however subsequently developed increasing respiratory distress warranting intubation and ICU admission. ECG showed LBBB and ST changes, troponin was mildly elevated and chest radiography showed bilateral airspace opacification. Bedside echocardiography revealed left ventricular apical ballooning due to basal hyperkinesis and apical hypokinesis, characteristic of TCM. Inotropic support and diuresis lead to rapid improvement in respiratory and haemodynamic status. She was extubated and rehabilitated on the ward. Formal echocardiography revealed $\mathrm{EF} 40 \%$ indicating cardiac function returned to previous baseline. Discussion: TCM is usually preceded by stress, however there is no consensus as to the mechanism of the stress response precipitating acute heart failure. Acute asthma is a rare physical stressor to consider in the differential. The challenge is distinguishing TCM from the asthma exacerbation itself or from acute coronary syndrome, which it mimics in terms of clinical, biochemical and electrocardiographic features, however there is no obstructive coronary lesion identified. Treatment is supportive, with resolution in weeks to months and rarely recurrence ${ }^{1}$.

Conclusion: This case demonstrates acute respiratory failure driving acute heart failure, highlighting the need for awareness of the relationship between asthma and TCM. 


\section{References:}

Gianni M, Dentali F, Grandi AM, Sumner G, Hiralal R, Lonn E. Apical ballooning syndrome or takotsubo cardiomyopathy: a systematic review. European Heart Journal. 2006;27(13):1523-9.

Sato H, Tateishi H, Uchida T, et al., Takotsubo type cardiomyopathy due to multivessel spasm. In: Kodama K, Haze K, Hon M, eds, Clinical aspect of myocardial injury: from ischemia to heart failure, Tokyo: Kagakuhyouronsya, 1990;56-64.

\section{A Case Of PPI-Induced Acute Interstitial Nephritis Without Recovery Of Renal Function In A Patient With Type 1 Diabetes Mellitus}

Connellan D, Redahan L

Dept. of Nephrology, Mater Misericordiae University Hospital, Eccles St, Dublin

\section{$\mathrm{DML} / \mathrm{UCD}$}

Introduction: Acute Interstitial Nephritis (AIN) was first described in 1898 by WT Councilman, chief pathologist in Brigham Hospital, and described as "non-suppurative inflammatory interstitial lesions", usually associated with streptococcal infection. ${ }^{1}$ Nowadays druginduced AIN accounts for $70 \%$ of cases; with common agents including antibiotics, NSAIDs, and increasingly, proton pump inhibitors (PPI). ${ }^{2}$

Case Description: A 29-year-old male with a history of type 1 diabetes mellitus and peptic ulcer disease (PUD) presented with a 1-week history of epigastric pain. His initial investigations noted a haemoglobin of $\mathrm{Hb}$ $4.5 \mathrm{~g} / \mathrm{dL}$ and a serum creatinine $(\mathrm{sCr})$ of $321 \mathrm{umol} / \mathrm{L}$. He was treated as pre-renal AKI secondary to anaemia with probable underlying diabetic nephropathy. His glycaemia control was poor from the outset with erratic sugars, frequently requiring IV insulin infusions.

His oesophago-gastro-duodenoscopy (OGD) revealed severe erosive oesophagitis with two oozing gastric and duodenal ulcers. He commenced treatment with an IV PPI and subsequently oral esomeprazole and lansoprazole.

By Day 10 of his admission, his $\mathrm{sCr}$ had increased to $500 \mathrm{umol} / \mathrm{L}$ and a renal biopsy revealed an eosinophil rich tubulointerstitial infiltrate, tubular necrosis and moderate to severe and occlusive arteriolarsclerosis.

His PPI was withdrawn but steroid therapy was deemed inappropriate given his severe PUD and poor glycaemic control. His renal function continued to worsen to creatinine $>600 \mathrm{umol} / \mathrm{l}$ with the eventual requirement of haemodialysis. He remains dialysisdependent 6-months following his initial presentation and is undergoing work-up for Simultaneous Pancreas-Kidney (SPK) transplant. Discussion: Progression to end-stage kidney disease (ESKD) without recovery of renal function in AIN is rare and may be more likely in patients with severe tubulointerstitial disease on biopsy. This is possibly reflective of pre-existing inflammatory or fibrotic changes related to underlying renal disease. ${ }^{3}$

\section{References:}

Raghavan, R. and Eknoyan, G. (2014). Acute interstitial nephritis; a reappraisal and update. Clinical Nephrology, 82(09), pp.149-162.

Muriithi, A. et al (2014). Biopsy-Proven Acute Interstitial Nephritis, 1993-2011: A Case Series. American Journal of Kidney Diseases, 64(4), pp.558-566

Clarkson, M. et al (2004). Acute interstitial nephritis: clinical features and response to corticosteroid therapy. Nephrology Dialysis Transplantation, 19(11), pp.2778-2783.

\section{Serial Bloodstream Infections Culminating In A CPE Bacteraemia}

Edwards Murphy D, Joyce E, Murphy M, Fleming C Dept. of Infectious Diseases, University Hospital Galway
WNW/NUIG

Introduction: Carbapenemese producing enterobacterales (CPE) are the latest superbug to threaten patient safety. Carbapenemeses are enzymes that breakdown broad-spectrum antibiotics such as meropenem, promoting antimicrobial resistance. Widespread hospital screening identifies colonisation; it is a reportable disease and mandates the isolation of patients with contact precautions and approximately $8 \%$ will develop a CPE infection from colonization. In this case we report the rare, but most serious, complication of colonisation a case of CPE bacteraemia, which represents a $2 \%$ risk once colonised.

Case Discussion: A 79-year old lady was admitted with a background of recurrent urosepsis requiring treatment with meropenem. Previous admission screening confirmed this patient was MRSA, VRE and CPE colonized. Meropenem was commenced on admission; blood and urine cultures then grew VRE and Daptomycin was then added. Further blood cultures grew a candida albicans and antifungal therapy was started. The source of both bloodstream infections was a hydronephrosis secondary to a renal stone; a percutaneous nephrostomy was performed aiming for source control. Despite this intervention this lady developed a third bloodstream infection, an OXA-48 carbapenemase producing Klebsiella pneumoniae on the seventeenth day of admission. The isolate was sensitive to ceftaz-avibactam and ciprofloxacin but resistant to meropenem. Interestingly OXA-48 isolates are often sensitive to meropenem but that was not the case here. This patient improved clinically following removal of the stone and source of urosepsis; antimicrobials were further rationalised to complete a 14 day course of ceftaz-avibactam and ciprofloxacin combination therapy. The patient was discharged home well.

Conclusion: Source control was critical in managing this patient with multiple bloodstream infections. In Ireland, CPE bacteraemia is rare compared with other EU constituents where it accounts for half of all bloodstream infections, with $50 \%$ mortality. Antimicrobial stewardship and hand hygiene are key measures to preventing CPE outbreak.

Nota Bene: An Audit Of The Medical Notes Of Surgical Patients

Noone A*, Troy A*, Ni Sheaghdha A, Burke $\mathrm{O}^{1}$, O’Sullivan R, Cullen N, O'Connor A, McNamara D

Dept. of Colorectal Surgery, Beaumont Hospital, Dublin

Joint First Authors*

\section{DNE/RCSI}

Objective: To perform an audit of the daily medical notes written by the primary team in charge of colorectal surgery patients' care and document any incidences of missing medical data.

Introduction: Note-taking is an important part of ensuring excellence of patient care. Accurate and encompassing documentation by the primary medical team in charge shows acknowledgement of the daily variances in their patient's medical conditions. It also allows for swift handover of information to healthcare workers unfamiliar with the patient and provides legal protection to practitioners in cases of litigation. However, the fast-pace of surgical ward rounds, the large volumes of patients, and the ever-changing aspects of the surgical patient's clinical status places doctors at a certain risk of forgetting to document all the relevant details of their patients.

Methods: We sought to evaluate this risk by examining the medical notes of colorectal surgery patients made by the primary team from the morning ward round, and then documenting the incidences of missing information that plays an important role in the daily care of the patient.

Results: There is often poor documentation of numerous important aspects of patient care. This does not provide adequate enough recordkeeping by us as practitioners and puts us potentially at risk in any future cases of litigation.

Conclusion: The introduction of a tailored document-checklist completed at the bedside may play an important role in decreasing these incidences of improper documentation. When utilised properly, this would 
help decreasing the time spent writing medical notes and improve the clarity and quality of record-keeping. We propose the introduction and implementation of such a document as a routine part of medical notetaking.

\section{Management Of High-Risk Cutaneous Squamous Cell Carcinoma At Galway University Hospital}

\author{
Cinelli D. O’Donnell J. Sheil F, Jones D \\ Plastic Surgery Dept., University Hospital Galway, Galway
}

\section{WNW/NUIG}

In the Irish population, Fitzpatrick Type 1 skin is most common, predisposing to non-melanoma skin cancers, such as squamous cell carcinomas. While complete surgical excision is the only treatment required for the majority of SCCs, an estimated 5-10\% of lesions have features associated with increased propensity to metastasise and are therefore considered high-risk lesions. The SIGN 140 NHS guidelines provide criteria for defining high-risk lesions, stating that such cases benefit from MDT discussion and in some cases, adjuvant therapy. This study aims to assess the frequency of such high-risk lesions in our department and to examine our practises in relation to MDT discussion and adjuvant treatment of such lesions.

The histopathology reports and online patient records relating to all patients who had a cutaneous SCC excised in July 2019 in our department were analysed. Histopathological data was used to identify those in the high-risk subgroup. MDT discussion, referral for radiotherapy and further surgical excisions, was recorded for these cases.

Of the 67 primary cutaneous SCCs excised, $69 \%$ had high-risk features. $67 \%$ of high-risk SCCs were located on the ear, nose or scalp, $20 \%$ of high-risk SCCS were discussed at MDM. $11 \%$ of these were re-excised, $78 \%$ underwent radiotherapy and $11 \%$ were followed for surveillance. $15 \%$ of patients received further treatment without multidisciplinary input, $71 \%$ undergoing re-excision and $29 \%$ undergoing radiotherapy. High-risk SCCs may be over-represented in our patient population. While all patients with cutaneous SCCs are followed clinically, the majority are not referred for adjuvant treatment or discussed at MDT. This study highlights the need for a standardised care model for lesions classed as high risk at a local, and national level.

\section{A Prospective Audit On The Incidence Of Urinary Catheter Related Urethral Injuries In Beaumont Hospital}

Meenan A, Nic an Ríogh A, Davis N

Urology Dept. TUN Directorate, Beaumont Hospital.

\section{DNE/RCSI}

Objective: To perform a prospective audit of the urethral injuries relating to urinary catheterisation in Beaumont Hospital over a 3-month period. Introduction: Urinary catheterisation is one of the most commonly performed procedures in medicine. To highlight the dangers associated with traumatic urethral catheterisation we prospectively monitored the incidence and clinical outcomes of urethral catheter related injuries

Methods: This prospective study was performed in Beaumont Hospital from July 2019 to September 2019. Data was collected daily as part of the Urology on-call handover sheet. Recorded data included method and extent of urethral catheterisation injury, urological management provided and complications associated with urinary catheter related trauma.

Result: A total of 13 catheter related injuries were recorded in male patients during this 3-month audit period. The mean patient age was 68.4 years. Urethral injuries were caused by creating false passages with the catheter tip in the urethra $(n=6)$ and by inflating the catheter anchoring balloon in the urethra $(n=7)$. To date, 3 of these patients have undergone further urological intervention as a result of their injuries, with two patients requiring cystoscopy and a third requiring an indwelling suprapubic catheter.

Conclusion: Current forms of educational training are insufficient for preventing urinary catheter related injuries. Iatrogenic urethral injuries will continue to occur unless improved training protocols are implemented or the safety mechanics of the urinary catheter are improved.

\section{Case Report: Systemic Al Amyloidosis Presenting As Acute Heart Failure}

McGuckin M, Murphy L, Joyce E

Dept. of Cardiology, Mater Misericordiae University Hospital, Dublin

\section{$\mathrm{DML} / \mathrm{UCD}$}

We report the case of a 46-year-old transgender female who presented with NYHA class IV symptoms with orthopnoea, paroxysmal nocturnal dyspnoea and a dry cough. The patient was an ex-smoker and is transgender, not currently on hormonal treatment, with no other past medical history. Clinical examination revealed an elevated JVP, bilateral crepitations and peripheral oedema. Brain natriuretic peptide was elevated at 900 and liver function tests were also deranged. ECG showed sinus rhythm with low voltage QRS complexes. Echocardiogram demonstrated biventricular failure, with an ejection fraction of $40-45 \%$ and severe left ventricular hypertrophy. Right heart pressures were elevated with severely reduced cardiac output.

Cardiac MRI showed a restricted left ventricle with diffuse subendocardial enhancement, in keeping with an infiltrative cardiomyopathy. Further investigations revealed elevated serum free light chains with a kappa predominance, and bone marrow plasmacytosis, diagnostic of multiple myeloma. Cardiac biopsy confirmed the diagnosis of AL amyloid cardiomyopathy, and treatment was initiated with bortezomib and dexamethasone.

The patient subsequently presented with syncope and a permanent pacemaker was implanted for suspected bradyarrhythmia. She subsequently presented with a PEA arrest and had a prolonged intensive care unit admission. She was successfully extubated, however, due to the severity of her illness is not a candidate for mechanical circulatory support or heart transplantation. She is now being treated in a palliative capacity with weekly bortezomib.

Discussion: Cardiac involvement is seen in $50-70 \%$ of patients with AL amyloidosis (1). Patients may present with heart failure caused by restrictive cardiomyopathy, arrhythmias due to conduction system involvement, or ischaemic heart disease due to amyloid deposition in coronary arteries. Median survival is less than 8 months (2), with most patients unsuitable for cardiac transplantation, often due to the extent of extracardiac involvement (3).

AL amyloidosis is a rare cause of restrictive cardiomyopathy with significant bradyarrhythmic consequences, as demonstrated in this case.

\section{References:}

Uptodate.com. (2019). UpToDate. [online] Available at: https:// www.uptodate.com/contents/cardiac-amyloidosis-clinical-manifestationsanddiagnosis? search $=$ cardiac\%20amyloid\&source $=$ search_result\&selectedTitle $=1 \sim 77 \&$ usage type $=$ default $\&$ display_rank $=1$ [Accessed 7 Dec. 2019].

Shi, J., Guan, J., Jiang, B., Brenner, D.A., del Monte, F., Ward, J.E., Connors, L.H., Sawyer, D.B., Semigran, M.J., Macgillivray, T.E. and Seldin, D.C., 2010. Amyloidogenic light chains induce cardiomyocyte contractile dysfunction and apoptosis via a non-canonical p38 $\alpha$ MAPK pathway. Proceedings of the National Academy of Sciences, 107(9), pp.4188-4193.

Dubrey, S.W., Cha, K., Anderson, J., Chamarthi, B., Reisinger, J., Skinner, M. and Falk, R.H., 1998. The clinical features of immunoglobulin light-chain $(\mathrm{AL})$ amyloidosis with heart involvement. QJM: monthly journal of the Association of Physicians, 91(2), pp.141-157. 


\section{New Mother New Diagnosis - A Case Report}

Egan M, Hussein $\mathrm{H}$

Dept. of Cardiology, Beaumont Hospital, Dublin

\section{DNE/RCSI}

Introduction: Spontaneous coronary artery dissection (SCAD) is defined as dissection of the epicardial coronary vessels not linked to atherosclerosis, trauma or cardiac catheterisation. ${ }^{1}$ SCAD is a rare illness often misdiagnosed, that accounts for up to $4 \%$ of cases of acute coronary syndrome (ACS) in young women. ${ }^{2}$

Case Presentation: A previously well 41 -year-old female presented to Beaumont Hospital with severe sudden onset central chest pain that radiated down her left arm and between both scapulae. She felt lightheaded, dyspnoeic and diaphoretic with one episode of vomiting at the onset of symptoms. Fourteen weeks prior, she had an uneventful spontaneous normal vaginal delivery. On examination she was tachycardic but otherwise vitally stable. $1^{\text {st }}$ and $2^{\text {nd }}$ ECGs showed an accelerated idioventricular rhythm with global ST elevation. The third ECG showed non-sustained VT with fusion beats and AV dissociation. The only abnormal blood result was troponin rise of 59 to 1749 .

Differential diagnosis included postpartum Takotsubo cardiomyopathy or coronary artery vasospasm. ECHO showed an estimated ejection fraction of $50 \%$ and an akinetic apex with basal and mid anterior wall hypokinesis. Coronary angiogram confirmed an extensive spontaneous dissection from mid to proximal LAD with TIMI 3 flow to distal vessel with no loss of side branches. Recommendations were for conservative medical management. Discussion: Coronary angiography is necessary to classify SCAD, which guides management. SCAD is often associated with underlying conditions such as fibromuscular dysplasia, connective tissue disease and vasculitis. Stressful events such as pregnancy or labour can also precipitate the condition. ${ }^{3}$ SCAD is the most common cause of MI related to pregnancy but pregnancy related SCAD accounts for less than $5 \%$ of all reported SCAD. ${ }^{4}$ The association is not well-understood. It was postulated that it is related to hormonal changes during pregnancy and the changes in the connective tissue and arterial walls. ${ }^{5}$

References:

1. Hayes SN, Kim ESH, Saw J, et al. Spontaneous Coronary Artery Dissection: Current State of the Science: A Scientific Statement From the American Heart Association. Circulation 2018; 137(19): e523-e557. 2. Al-Sadawi M, Shaikh S, Marmur JD, Salciccioli L, Kariyanna PT, McFarlane IM. Post-partum Spontaneous Coronary Artery Dissection: A Case Report. Am J Med Case Rep. 2018;6(10):218-221.

3. Puri R, Leong DP, Nicholls SJ, Liew GY, Nelson AJ, Carbone A, Copus B. et al. Coronary artery wall shear stress is associated with endothelial dysfunction and expansive arterial remodelling in patients with coronary artery disease.EuroIntervention 2015; 10(12): 1440-1448

4. Ito H, Taylor L, Bowman M, Fry ET, Hermiller JB, Van Tassel JW. Presentation and therapy of spontaneous coronary artery dissection and comparisons of postpartum versus nonpostpartum cases. Am J Cardiol 2011. June 1; 107(11): 1590-6

5. Sheikh AS, O’Sullivan M. Pregnancy-related Spontaneous Coronary Artery Dissection: Two Case Reports and a Comprehensive Review of Literature. Heart Views: The Official Journal of the Gulf Heart Association 2012; 13(2): 53-65.

\section{An Unusual Cause Of Bowel Obstruction}

Ivers A $\mathrm{i}^{\mathrm{i}}$, Toor A, Aremu MA

Dept. of Surgery, Connolly Hospital Blanchardstown, Dublin

\section{DNE/RCSI}

We report the case of a 25-year old Indian gentleman who presented to the emergency department with a 2-week history of generalised abdominal pain and vomiting, associated with night sweats and weight loss of $10 \mathrm{~kg}$ over 4 months. Physical examination revealed abdominal distension, tenderness and guarding, with increased bowel sounds. He had no medical or surgical history but family history was notable for tuberculosis 16 years prior.

Plain abdominal radiograph showed dilated loops of small bowel and computed tomography confirmed small bowel obstruction, with matted bowel loops, extensive irregular mural thickening, moderate volume ascites, omental thickening and nodular peritoneal deposits. Diagnostic laparoscopy showed widespread peritoneal, omental and small bowel white plaque deposits. Adhesions to the anterior abdominal wall were divided, biopsies were taken from the omental and peritoneal deposits and ascitic fluid sample was sent for culture, ZN stain and cytology.

The patient recovered well postoperatively. Mantoux test returned positive, although was not sufficient to diagnose current TB infection. Histological examination revealed caseating granulomas, however, there were no acid-fast bacilli seen on microscopy and microbiological cultures failed to grow mycobacteria. Nucleic acid amplification testing (NAAT) using PCR was subsequently performed on ascitic fluid samples and was positive for Mycobacterium tuberculosis.

Chest radiographs showed no signs of pulmonary involvement. Public Health were notified and concurrent HIV testing returned negative.

Anti-TB quadruple therapy was commenced for minimum of six months, with liver, renal and ophthalmology baseline assessments performed. Abdominal TB is a rare manifestation of extra-pulmonary TB (EPTB). Traditional methods of diagnosis are based on sputum samples and are highly likely to be negative in EPTB. When suspected, it is recommended to use NAAT and start empiric therapy if there is a high clinical suspicion of infection.

\section{Angiosarcoma In A Previously Irradiated Breast: A Case Report}

O'Flaherty C, Alazawi D

Dept. of Breast and General Surgery, St. James's Hospital Dublin

\section{DSE/TCD}

Introduction: Radiation exposure is a known risk factor for soft tissue sarcomas. Radiotherapy associated angiosarcoma (RAAS) has been reported as a rare but significant late complication of breast irradiation used as part of the treatment for breast cancer ${ }^{1}$.

Case presentation: Ms. X, a 75-year old lady, was referred to the triple assessment clinic in November 2019 with a one-month history of a right breast lump. This occurred on a background of previous right breast invasive ductal carcinoma in 2008 for which she underwent wide local excision and axillary node clearance followed by a course of adjuvant chemotherapy and radiotherapy and left breast ductal carcinoma in situ in 2013 for which she underwent wide local excision followed by radiotherapy.

Clinical examination noted a lump in the medial aspect of the right breast graded E5 as per the BIRADs scoring system. She underwent a same day mammogram and ultrasound which showed the presence of a $3 \mathrm{~cm}$ heterogeneous mass with calcifications correlating with the area of clinical concern and was graded R4. There were no pathological nodes. The impression at the time was of a high grade invasive ductal carcinoma however a core biopsy was taken which confirmed the presence of a rarer, more aggressive malignancy - an angiosarcoma.

Subsequent staging investigations including CT TAP and bone scan were negative. Her case was discussed at the breastcare multidisciplinary team meeting and it was decided to proceed with surgical management involving a right mastectomy.

Discussion: In the largest single cohort study to date the risk of RAAS following radiotherapy is approximately $0.1 \%{ }^{2}$. Due to its rarity defining optimal treatment is challenging. Surgery, radiotherapy and chemotherapy have been proposed with surgery remaining largely the standard of care to date. Despite all treatments however RAAS is an aggressive malignancy associated with a high mortality ${ }^{3}$.

\section{References:}

Huang J, Mackillop WJ. Increased risk of soft tissue sarcoma after radiotherapy in women with breast carcinoma. Cancer 2001; 92:172-180 
Rombouts AJM, Huising J, Hugen N, et al. Assessment of RadiotherapyAssociated Angiosarcoma After Breast Cancer Treatment in a Dutch Population-Based Study. JAMA Oncol. 2019;5(2):267-269

Torres KE, Ravi V, Kin K, Yi M, Guadagnolo BA, May CD, et al. Longterm outcomes in patients with radiation-associated angiosarcomas of the breast following surgery and radiotherapy for breast cancer. Ann Surg Oncol. 2013;20(4):1267-74.

\section{Ultrasonography In Acute Appendicitis In Paediatrics - The Diagnostic Yield}

Farouk A, Devine M, Merrigan A, Tormey S, Buckley J

Dept. of Breast Surgery, University Hospital Limerick

\section{MW/UL}

Background: The most common cause of emergency surgery in the paediatric population is acute appendicitis. Ultrasonography remains the standard initial radiological investigation of choice in diagnosis of acute appendicitis in children although significant variation in efficacy exists within different health centres. The objective of this study was to explore the diagnostic value and limitations associated with ultrasonography in acute appendicitis in a paediatric cohort while assessing the specificity and sensitivity. A secondary objective of the study was to evaluate the negative appendicectomy rates in this tertiary referral centre.

Methods: A retrospective study of all paediatric patients who underwent appendicectomy between the ages of 4 and 16 that had pre-operative ultrasounds from $01 / 01 / 2017$ to $31 / 12 / 2018$ was carried out in University Hospital Limerick. The Health InPatient Enquiry system and hospital radiology software were used to identify the required patients with assessment of clinical, radiological, intra-operative findings, and histological results.

Results: A total of 341 patients had an appendicectomy over the 24month period, 119 patients had pre-operative ultrasounds. Of these, following histopathology confirmation, ultrasonography was found to have a sensitivity of $36.67 \%$ and specificity of $98.31 \%$ with a positive predictive value of 0.95 and negative predictive value of 0.60 . One patient was found to be falsely positive on US.

Conclusion: Though highly specific for appendicitis, the utility of obtaining a pre-operative ultrasound is low given the undesirable sensitivity and wide user variation, hence treatment in accordance with astute clinical examination is paramount.

Fast Positive! Assessing Clinical Information Provided By Doctors For Thrombolysis Alerts And Comparing To Diagnostic Outcome

Davey $\mathrm{MG}^{1}$, Waters $\mathrm{C}^{1}$, Moran, $\mathrm{L}^{1}$, Dennehy, $\mathrm{K}^{1}$, O’Malley $\mathrm{E}^{1}$, McCarthy, $\mathrm{P}^{1}$, Walsh $\mathrm{T}^{2}$

Depts. of Radiology ${ }^{1}$, Geriatric Medicine ${ }^{2}$, University Hospital Galway

\section{WNW/NUIG}

Background: FAST positive stroke calls rely on efficient and detailed communication between health care professionals to provide optimal patient outcomes. Our primary goal was to improve the quality of clinical information provided by doctors on imaging requests for thrombolysis alerts to improve stroke call outcomes.

Methods: 100 consecutive FAST positive stroke cases were included in our initial assessment (IA) between April - August 2018. Clinical information provided in the initial imaging request was assessed and compared with the final radiological diagnoses. 'Clinical information' provided on imaging requests and 'resulting diagnosis' were ranked in two categories; (1) Bamford classification stroke syndrome, and (2) the side of the suspected stroke. A quality improvement initiative was carried out; study results were communicated to the NCHD lead and consultant-led thrombolysis training was held for medical registrars. Our second assessment (SA) involved 75 cases assessed between January-July 2019.
Results: There were 100 cases in the IA and 75 cases in the SA. 79\% $(n=79)$ provided sufficient clinical information to correspond to a stroke syndrome and specified a side of symptoms/territory in IA, whilst $64 \%$ $(\mathrm{n}=48)$ did in SA. In IA, 5 cases provided no clinical information; for example "FAST +" and "Stroke Alert". 3 cases provided no clinical information in SA. $45 \%$ of cases in IA had a resulting diagnosis corresponded to the stroke syndrome in the request, and $73 \%$ had in the SA. The IA had the resulting side of lesion corresponded to the side/territory in $73 \%$, with only $59 \%$ in the SA.

Conclusion: Thrombolysis alerts are time sensitive scenarios where accurate detail and efficient communication are paramount to provide the best outcomes. Our quality improvement intervention provided modest improvement in patient outcomes in our tertiary referral centre. This study highlights the requirement for further training and quality improvement initiatives to enhance thrombolysis call outcomes.

An Audit On Management \& Outcomes Of Adrenal Incidentalomas Detected On CT Scans

Haque $\mathrm{M}^{1}$, Ramesh $\mathrm{N}^{1}$, Kyithar $\mathrm{MP}^{2}$

Depts. of Radiology ${ }^{1}$, Endocrinology ${ }^{2}$, Midlands Regional Hospital Portlaoise

$\mathrm{DML} / \mathrm{UCD}$

Background: An adrenal incidentaloma (AI) is defined as any adrenal mass $\geq 1 \mathrm{~cm}$ in its longest axis that is detected incidentally on $\mathrm{CT}^{1}$. AIs are either secretory or non-secretory, benign or malignant, so further evaluation is warranted with laboratory findings and $\mathrm{CT}$ with and without contrast to distinguish characteristics of AIs. As AIs have become a common clinical dilemma with the increasing use of CT, investigations need to be carefully chosen to prevent unnecessary and/or expensive tests whilst simultaneously preventing underdiagnosis of a secreting or malignant tumour.

Aims: This retrospective audit examined the assessment of AIs in MRHP and its adherence to European Society of Endocrinology (ESE) Clinical Practice guidelines ${ }^{2}$.

Methods: Reviewing 3749 abdominal CT scans between $1^{\text {st }}$ January $31^{\text {st }}$ December 2018 in MRHP \& identifying an incidental adrenal lesion. Chart review was conducted $\&$ an anonymised database was created to identify if follow up recommended by guidelines ${ }^{2}$ was adhered to.

Results: 129 patients with adrenal lesions were identified; $50.4 \%$ male \& $49.6 \%$ female; $29 \%$ were referred to endocrinology clinics; $56.6 \%$ were adrenal adenomas $>1 \mathrm{~cm}$ (not incll patient with an adenoma of $9 \mathrm{mmx} 8 \mathrm{~mm}$ ), $27.1 \%$ were bulky adrenals; $15.5 \%$ were adrenal nodules; $69.8 \%$ were left sided, $15.5 \%$ were right sided, $14.7 \%$ of which were bilateral adrenal lesions. $15 \%$ received follow up MRI/non-contrast CT adrenals; $78 \%$ received no biochemical investigations, however in $11 \%$ of them, co-morbidities such as metastatic disease \& palliative status precluded them from further investigation. By contrast, all patients reviewed by the Endocrinology team were appropriately investigated and managed; the majority conservatively, including a malignant phaeochromocytoma due to advanced age, and none required surgical intervention.

Conclusion: The management of AI in MRHP reflects poor adherence to guidelines. Adherence was significantly better in patients managed by the Endocrinology team. We suggest the development of a local referral \& management pathway.

References:

Menegaux, F., Chéreau, N., Peix, J.-L., Christou, N., Lifante, J.-C., Paladino, N., ... Mathonnet, M. (n.d.). Management of adrenal incidentaloma. Journal of Visceral Surgery, 151(5), 355-364.

Fassnacht, M., Arlt, W., Bancos, I., Dralle, H., Newell-Price, J., Sahdev, A., Tabarin, A., Terzolo, M., Tsagarakis, S., \& Dekkers, O. (2016). Management of adrenal incidentalomas: European Society of Endocrinology Clinical Practice Guideline in collaboration with the European Network for the Study of Adrenal Tumors, European Journal of Endocrinology, 175(2), G1-G34. 
A Cross Sectional Study Illustrating The Clinical Utility Of An Admission Chest Radiograph In An Acute Stroke Population

Murphy F, Harris L, Rutherford R

Respiratory Dept., Galway University Hospital Group

\section{WNW/NUIG}

Background: Usefulness of chest radiographs in the acute stroke setting is unclear, with recent guidelines recommending it as a Class $2 \mathrm{~b}$ recommendation. We sought to evaluate patterns of acute CXR acquisition in an acute stroke population in an Irish emergency department and its clinical utility.

Methods: This was a retrospective observational study. We reviewed all patients presenting with acute stroke syndromes who had a CXR performed during their inpatient stay. Admission CXRs, and any subsequent CXRs performed were reviewed.

Results: We examined 220 patients over a 12-month period. $77.3 \%(n=$ 170) had a CXR performed on the day of admission. $53.5 \%$ of these admission CXRs were reported as normal. Radiographic abnormalities included pulmonary nodules, consolidation, cardiomegaly, atelectasis, and pulmonary oedema. There were 7 patients with new pulmonary nodules. One was diagnosed with lung cancer, and two patients were diagnosed with pulmonary metastases from separate primary tumours. $38 \%$ $(n=19)$ of the patients without an admission CXR had one performed during their inpatient stay, with $68 \%(\mathrm{n}=13)$ of these CXRs having a radiological abnormality.

Conclusion: A routine CXR on admission had high clinical utility. As an opportunistic screening method in a stroke population it is high yield for picking up suspicious lung lesions. It must not delay door to scanner time but has the potential to be useful in an acute setting.

Verotoxigenic E.Coli Resulting In Haemolytic Uraemic Syndrome (HUS): A Case Report

Hulston S, Palmer C, Sheridan J

Dept. of Gastroenterology, St. Vincent's University Hospital, Dublin

\section{$\mathrm{DML} / \mathrm{UCD}$}

Introduction: Infection with E.coli 0157 can present with a variety of symptoms including bloody diarrhoea and abdominal cramps. This infection is often linked with HUS. ${ }^{1}$ HUS is a clinical syndrome characterized by the triad of thrombotic microangiopathic haemolytic anaemia, thrombocytopenia, and acute kidney injury. ${ }^{2}$ Supportive therapy remains the mainstay of treatment.

Case Details: A 20-year-old female presented to the emergency department with a three-day history of bloody diarrhoea, abdominal cramps and vomiting. The cramps were worse on passing stool and were a 7/10 in pain. She was apyrexial on admission and reported no subjective fevers. She had no history of recent travel or sick contacts. On admission, her bloods were grossly normal with only a mildly elevated CRP. Differential diagnoses included infective gastroenteritis and new diagnosis of IBD. A stool sample was sent for evaluation and sigmoidoscopy was performed. Sigmoidoscopy showed severe inflammation extending from the proximal descending colon to the sigmoid, with marked oedema and ulcer-like lesions. The stool was positive for Verotoxigenic E.coli 0157. The public health department was notified. The patient was isolated and treated conservatively with IV fluids and antibiotics were avoided. She remained stable and was discharged with verbal advice. Unfortunately, six days after discharge, RK was readmitted with persistent nausea and vomiting, epigastric pain and two episodes of haematemesis. Her diarrhoea had resolved, and she was passing stool and urine normally. This time she was anaemic $(\mathrm{Hb}=5.6)$, thrombocytopaenic (platelets=115) and had a severe AKI (Urea=25.7 and Creatinine=165). She was diagnosed with
HUS and taken over care by the renal team. She was transfused two units of red cells and managed conservatively.

Discussion: This case highlights how it can be difficult to avoid the progression of VTEC to HUS. Even though antibiotics were avoided, and the patient was stable on discharge, she still developed this complication.

References:

1. Su C, Brandt LJ. Escherichia coli O157: H7 infection in humans. Ann Intern Med. 1995;123:698-707. https://doi.org/10.7326/0003-4819-1239-199511010-00009.

2. Canpolat, N. (2015). Hemolytic uremic syndrome. Türk Pediatri Arşivi, 50(2), pp.73-81.

Anti-Phospholipid Syndrome: An Unusual Cause Of Deranged Liver Function Tests

Moynagh N, O'Shea B

Rheumatology Dept., St. James's Hospital Dublin

\section{DSE/TCD}

Introduction: Antiphospholipid syndrome is a systemic autoimmune disorder with a vast array of clinical presentations. It is characterized by venous or arterial thrombosis, in the presence or absence of pregnancy morbidity, with persistent laboratory evidence of antiphospholipid antibodies. (1) Method: A case report on Mrs. X, a thirty-six-year old woman who presented to St. James's hospital with deranged liver function tests (LFTs) and severe epigastric on a background of chronic atrophic pancreatitis

Results: Mrs. X presented to St. James's Hospital with severe epigastric pain, deranged LFTs and high blood glucose on a background of chronic pancreatitis secondary to gallstones. CT abdomen showed gross abdominal distention and a head of pancreas mass, querying a tumour and subsequent tumour thrombus leading to her presentation. Gastroenterology suggested an upper gastro-intestinal scope with potential subsequent endoscopic retrograde cholangiopancreatography, advising that current LFTs would be in line with an obstructing stone. When her upper gastrointestinal scope was normal, they progressed to endoscopic ultrasound (EUS). EUS showed extensive portal vein thrombosis. No suspicious head of pancreas mass was identified. MRI pancreas was advised to further classify findings, along with a prompt haematology review. MRI pancreas showed propagation of the thrombus into multiple segmental branches of the liver. When worked up by the haematology service, it was noted that Mrs. X was lupus anticoagulant positive. With the clinical criteria of arterial thrombosis and the first of two required antibodies testing positive, a working diagnosis of anti-phospholipid syndrome was made. Mrs. X was initiated on therapeutic enoxaparin to prevent further clot propagation. She will receive close follow up by our coagulation and hepatology services for the foreseeable future.

Conclusion: Anti-phospholipid syndrome is a differential for a multitude of presentations. Prompt diagnosis and management is essential for best patient outcome.

\section{Reference:}

(1) Doruk E, et al. (2019) Diagnosis of antiphospholipid syndrome. Uptodate. https://www.uptodate.com/contents/diagnosis-ofantiphospholipid-syndrome? search=anti\%20phosphol ipid $\% 20$ syndrome \& source $=$ search result\&selectedTitle $=1 \sim 150 \& u$ sage_type $=$ default\&display_rank $=\overline{1} \# H 72934381$

\section{Recurrent Collapse When 26 Weeks Pregnant: A Diagnostic Dilemma}

Mulligan M, Delanty N

Epilepsy Monitoring Unit (EMU), Beaumont Hospital, Dublin

\section{DNE/RCSI}


Case: A 30-year-old female was admitted to the Epilepsy Monitoring Unit following a history of recurrent collapse with suspected seizurelike activity over the previous 14 years. A typical event occurred when waking from sleep; she experienced a prodrome of 'tingling' in her face, headache and feeling flushed prior to collapsing from her bed. She typically lost consciousness and had whole-body shaking lasting approximately 2 minutes. No tongue biting or urinary incontinence were noted. She felt tired after the events. Since age 16, event frequency had been every 3 months, however, during the pregnancy, they had increased to monthly. Over the preceding years, she was reviewed by cardiology (normal ECG, exercise stress test, echocardiogram and cardiac MRI with sinus rhythm on Holter), neurology (no epileptiform discharges on EEG, normal CT and MRI brain) and endocrinology (Addison's and insulinoma outruled). As an isolated EEG could not outrule seizures, levetiracetam was commenced and videoEEG scheduled. During EMU admission, one subjectively typical event (similar to above) was recorded. On EEG review, no epileptiform discharges were noted throughout admission; however, cardiac telemetry being simultaneously recorded revealed earlymorning complete heart block with ventricular standstill. With ventricular standstill lasting between 3 and 7 seconds on occasion, a dual chamber permanent pacemaker was inserted. The patient was discharged for outpatient follow-up.

Discussion: This case demonstrates how collapse history and investigation, despite being extremely prevalent, can be challenging for clinicians to correctly identify the aetiology. The inter-specialty nature of the presentation requires specialists to remain cognisant of other diagnoses. Technology should be emphasised to patients as a useful tool, videoing the events for physician review can aid management. The sensitivity and specificity of investigations should be considered and if the history is non-conclusive, more thorough work-up should be expedited.

\section{Increasing Compliance With Diabetes Cycle Of Care In General Practice}

Carthy $\mathrm{E}^{1}$, O'Shea $\mathrm{E}^{2}$, Halloran $\mathrm{R}^{2}$, Howard $\mathrm{N}^{2}$, Cantillon $\mathrm{P}^{2}$ University College Hospital Galway ${ }^{1}$, West North West Intern Network Claddagh Medical Centre ${ }^{2}$, Galway

\section{WNW/NUIG}

Introduction: The increasing prevalence of diabetes mellitus, particularly type 2 , has led to the development of appropriate care through a more structured involvement of primary and secondary care sectors. The Diabetes Cycle of Care $(\mathrm{CoC})$ was introduced by the HSE in October 2015 to enable patients and physicians a streamlined bi-annual assessment of their condition. Literature has assessed specific areas of the cycle, yet limitations exist in exploring compliance with these guidelines and methods to improve patient-physician uptake.

Aim: To perform a single-centre audit on patient eligibility and compliance with the $\mathrm{CoC}$, and to implement a fail-safe recall system that can be reproduced nationally.

Methods: A standardised audit cycle was introduced over a 12-month period. A prospective audit database of patients with Type 2 Diabetes calculated the number of visits over the $1^{\text {st }}$ stage of the audit cycle. Practice staff were then educated on the data and a recall system was introduced as an intervention. A subgroup analysis was also performed on time since diagnosis, associated complications, social status and HbA1c.

Results: Of 104 patients with T2DM, 22\%(n=23) had no appointment in the given year, $55 \%(\mathrm{n}=59)$ had one while $23 \%(\mathrm{n}=24)$ had two or more. There was little gender discrepancy in non-attendees with $56 \%(n=13)$ male, and $43 \%(n=10)$ females. Average age of non-attendees was 61 while average age of total was 68.75 .
Conclusion: The primary outcome of this audit showed a low rate of patient-physician compliance with the $\mathrm{CoC}$ bi-annual review. This study was also able to identify positive predictive factors for non-attendance. Whilst this primary care centre is above the regional average, we hypothesise the intervention introduced will increase compliance to national expectations. To complete this audit cycle, we are currently reassessing the impact of the recall system with a view for this intervention to be utilised by other health centres.

\section{An Audit Of Diabetic Ketoacidosis Management In Naas General Hospital}

O’Byrne $\mathrm{R}^{1,2}$, Moore $\mathrm{K}^{1}$, Woods, $\mathrm{C}^{1}$

Naas General Hospital ${ }^{1}$, Naas, Kildare; Tallaght University Hospital ${ }^{2}$, Tallaght, Dublin

\section{DSE/TCD}

Diabetic ketoacidosis (DKA) represents the diabetic emergency defined by ketosis, acidosis, and hyperglycaemia. The physiological effects of DKA can be fatal if rapid treatment is not instigated, and precise clinical care is required to maximise the chances of recovery, while minimising the risks of complications. It is vitally important that institutions audit their management of DKAs to ensure treatment is line with local policies and international best practices. This audit studied DKAs in Naas General Hospital (NGH) over a oneyear period and assessed how well management guidelines were followed. In total, there were 22 instances of DKA, in 20 patients, with 2 cases occurring in inpatients. Infection was the single largest precipitant of DKA, particularly in those patients with type 1 diabetes mellitus (T1DM). The mean time to starting the hospital's DKA protocol was $2.1 \mathrm{hrs}$, with the mean length of protocol (total time on fixed-rate insulin infusion) being $19.8 \mathrm{hrs}$. Management and monitoring of patients were largely in-line with hospital protocol, particularly in terms of glucose and ketone monitoring (95\% adherence to protocol). $\mathrm{K}^{+}$monitoring was poor however, with only $5 \%$ of patients getting $\mathrm{K}^{+}$measurements as stipulated. The importance of regular $\mathrm{K}^{+}$measurements is highlighted here by the occurrence of hypokalaemia and hyperkalaemia in $36 \%$ and $27 \%$ of patients respectively; when detected, these complications were typically managed appropriately during this audit. Hypoglycaemia was found to occur in $27 \%$ of patients, with the hypoglycaemia protocol started appropriately in all of these cases. Resolution of DKA was confirmed in $68 \%$ in patients, while only $45 \%$ of patients were transferred to SC insulin as per protocol. While is scope for improvement in DKA management in NGH, overall the findings of this audit compared favourably to similar audits performed elsewhere.

Chest Pain And Wellens Pattern ECG In A Patient With Marfan Syndrome: The Diagnostic Challenge

O’Leary A, Hynes B

Dept. of Cardiology, Galway University Hospital

\section{WNW/NUIG}

Introduction: Marfan Syndrome (MFS) is a connective tissue disorder of the fibrillin 1 gene with an incidence of 1/3000-5000. Given multiple cardiac manifestations, including aortic regurgitation, mitral valve prolapse, aortic dilatation and dissection, presentation with chest pain can prove a significant diagnostic challenge.

Case Presentation: A 53-year-old lady with MFS and a known $3.9 \mathrm{~cm}$ ascending aortic aneurysm was admitted with a 10-day history of retrosternal chest pain radiating to the back and left arm, associated with one episode of right acrocyanosis. Cardiovascular examination was unremarkable with features of MFS, including arachnodactyly, noted. 
Electrocardiogram revealed Wellens pattern type 1, pathognomonic of critical proximal LAD stenosis. Serial data revealed Troponin-Tn of 69 and 58 at hours zero and six respectively. All other bloods were unremarkable. A working diagnosis of an NSTEMI was made. Due to the presence of a known thoracic aortic aneurysm, a dissection had to be excluded prior to administration of dual anti-platelet therapy. An urgent gated CT-Aortogram was performed which excluded a dissection but revealed a calcific lesion of the proximal LAD. She proceeded to coronary angiogram where a $90 \%$ lesion of the proximal LAD was identified, confirming Wellens Syndrome. She had PCI performed and was discharged home symptomatically well the following day. Discussion: This case demonstrates the challenge posed by chest pain in the patient with MFS. Wellens pattern is associated with $75 \%$ progression to acute anterior MI necessitating urgent management. Typically, presence of a Wellens pattern and troponin leak in the absence of MFS would be diagnostic of a NSTEMI; however in this case of a patient with a known thoracic aortic aneurysm, we could not proceed with NSTEMI management without a CT-Aortogram.

\section{The Diagnostic Complexities Of Infected Hepatic Cysts}

Lowry $\mathrm{J}^{1}$, Murphy $\mathrm{M}^{1}$, Tuite $\mathrm{H}^{1}$, Ward $\mathrm{J}^{2}$

Depts. of Infectious Disease ${ }^{1}$, Radiology $^{2}$, University Hospital Galway

\section{WNW/NUIG}

Introduction: Cystic lesions of the liver represent a heterogenous class of disorders varying greatly in aetiology and clinical presentation. While predominantly asymptomatic and found incidentally, they can present with complications. Infected Hepatic Cysts represent a rare, yet severe complication associated with high morbidity and mortality. These infections are highly heterogenous, yet their aetiological distinction is essential as it influences patient management [1]. However, as highlighted by this case, this can be difficult to achieve in clinical practice.

Case: We present the case of an 81-year-old nursing-home-resident who presented with breathlessness and right-upper-quadrant pain over the past five days. On examination she was apyrexial, with tenderness in the rightupper-quadrant and massive hepatomegaly.

Blood tests showed significantly elevated inflammatory markers [CRP 556 $\mathrm{mg} / \mathrm{L}]$, elevated white-cells $\left[14.2 \times 10^{9} / \mathrm{L}\right]$ and an obstructive pattern of liver enzyme elevation. An ultrasound abdomen revealed a large complex hepatic cyst with a measured volume of $8.5 \mathrm{~L}$. This was followed by Computed Tomography revealing dimensions of $30 \times 24 \times 20 \mathrm{~cm}$ and an appearance concerning for hydatid aetiology - for which CT's sensitivity approaches 94\% [2]. Thus, treatment with Piperacillin-Tazobactam and Albendazole was commenced and drainage/aspiration delayed to a later date (due to associated risk of anaphylaxis in hydatid disease if leakage of cyst contents precedes Albendazole therapy).

Due to clinical deterioration in the following days, Percutaneous Transhepatic Drainage was performed and $8.5 \mathrm{~L}$ of fluid was drained. This was subsequently culture-positive for Escherichia-Coli, with culture and serology negative for Echinococcus.

Discussion: Clinical presentation coupled with imaging resulted in a highly suspected diagnosis of Hydatid Disease, however ELISA serology returned negative. Furthermore, some case series report $30-40 \%$ of patients with hepatic cystic echinococcosis as antibody negative [3]. Ultimately, while definitive percutaneous drainage was performed, this case highlights the complexity of hepatic cystic infection and the diagnostic uncertainty surrounding their aetiology.

\section{References:}

[1] Lantinga MA, Geudens A, Gevers TJG, Drenth JPH. Systematic review: the management of hepatic cyst infection. Alimentary Pharmacology \& Therapeutics. 2014Dec;41(3):253-61.

[2] Marrone G, Crino' F, Caruso S, Mamone G, Carollo V, Milazzo M, et al. Multidisciplinary imaging of liver hydatidosis. World J Gastroenterol. 2012;18:1438-1447.
[3] Zhang W., McManus D.P. Recent advances in the immunology and diagnosis of echinococcosis. FEMS Immunol Med Microbiol. 2006; 47(June (1)):24-41

A Combined Investigation Of Antepartum Information On Analgesia/Anaesthesia Techniques, And Patient Satisfaction Survey Regarding Same

Meagher, $\mathrm{A}^{1}$; Cribben, $\mathrm{N}^{1}$; Costello, $\mathrm{J}^{2}$. Anaesthesia Dept., ${ }^{1}$ University Hospital Galway; Saolta Hospital Group ${ }^{2}$

\section{WNW/NUIG}

Introduction: A key recommendation of the OAA / AAGBI Guidelines for Obstetric Anaesthetic Services 2013 was that 'Women should have antenatal access to evidence-based information about the availability and provision of all types of analgesia and anaesthesia'.

Comprehension is a cornerstone of informed consent and it is imperative that women have adequate knowledge regarding risks and benefits of analgesia and anaesthetic techniques prior to consent being obtained. Ideally, this information should be conveyed at multiple points during the antenatal course, to allow time for discussion and questions.

Methods: We conducted a post-partum survey of first-time mothers regarding their receipt of both oral and written information and satisfaction levels with same. Information sought included:

Primary source of information (Medical professionals, Booklets, Online Sources etc.) Attendance at antenatal classes

Overall satisfaction with information provided

Results:

$100 \%$ received antenatal information regarding analgesia/anaesthesia $66 \%$ received this in written form

$76 \%$ were "very satisfied", and overall satisfaction levels were at $90 \%$

$76 \%$ of respondents attended antenatal classes, and of this group, $87 \%$ were "very satisfied"

A third of women used the internet/friends/family as their primary source of information

Patients whose primary source of information was a medical professional also had higher satisfaction levels

Conclusions: Our results show a positive relationship between patient satisfaction and attendance at antenatal classes, as well as positively correlating with medical professionals as the primary source of information. We must continue to strive for high standards both in true informed consent, and patient satisfaction with our services.

Hyperparathyroidism And Panhypopituitarism - A Case Report Of An Elusive Endocrinological Diagnosis

McLaughlin E, O'Meara YM

Dept. of Nephrology, Mater Misericordiae University Hospital, Dublin

\section{$\mathrm{DML} / \mathrm{UCD}$}

Background: Pan-hypopituitararism is a rare condition, caused by pituitary tumours or their treatment, in which there is decreased secretion of most or all pituitary hormones. ${ }^{3}$

The incidence is 4.2 per 100,000 per year. $^{4}$

Case Report: We present the case of a 54-year old lady with end-stage renal disease secondary to diabetic nephropathy who presented to dialysis with a two month history of worsening malaise, fatigue, constipation and a fluctuating headache. Investigations revealed hypercalcaemia $(2.82 \mathrm{mmol} / \mathrm{L})$ and an elevated parathyroid hormone $(62.5 \mathrm{mmol} / \mathrm{L})$ despite being on a stable dose of Cinacalcet for secondary hyperparathyroidism. A Sestamibi scan confirmed parathyroid hyperplasia, in keeping with secondary hyperparathyroidism, and a parathyroid adenoma. It was felt that this was symptomatic hypercalcaemia and surgery was advised. The pre-operative course was complicated by 
fluctuating consciousness levels, resistant hypercalcaemia and haemodynamic instability, requiring CVVHD and inotropic support. However, post-parathyroidectomy, despite normalisation of calcium levels, symptoms of fluctuating consciousness and malaise persisted. An MRI Brain was sought and subsequently showed a pituitary macroadenoma. The pituitary screen showed panhypopituitarism, with a deficiency in all anterior pituitary hormones; most notably a morning cortisol of $32 \mathrm{nmol} / \mathrm{L}$ and simultaneous ACTH of $15 \mathrm{ng} / \mathrm{L}$. Intravenous hydrocortisone was started immediately, with a significant improvement of all symptoms within 24 hours. The patient was discharged on oral hydrocortisone and has been referred for consideration of pituitary surgery. Conclusions: Identification of the pituitary macroadenoma explained a lot of the patients symptoms. It prompted consideration that there may have been an infiltrating granulomatous process affecting the pituitary which had caused the initial sudden derangement in calcium homeostasis. ${ }^{5}$ However work-up for malignancy and granulomatous disease was negative. Ultimately, this was a non-functioning pituitary macroadenoma with mass effect and resultant panhypopituitarism as well as hypercalcaemia due to a parathyroid adenoma. There remains the consideration of a possible MEN syndrome, connecting the parathyroid and pituitary findings in this patient.

\section{References:}

(1) Tanriverdi F, Dokmetas HS, Kebapcı N, et al. Etiology of hypopituitarism in tertiary care institutions in Turkish population: analysis of 773 patients from Pituitary Study Group database. Endocrine 2014; 47:198. (2) Regal M, Páramo C, Sierra SM, Garcia-Mayor RV. Prevalence and incidence of hypopituitarism in an adult Caucasian population in northwestern Spain. Clin Endocrinol (Oxf) 2001; 55:735.

(3) Leporati P, Landek-Salgado MA, Lupi I, et al. IgG4-related hypophysitis: a new addition to the hypophysitis spectrum. J Clin Endocrinol Metab 2011; 96:1971.

\section{The Complications Associated With Hypertriglyceridemia: A Case Report}

Hanahoe A, Byrne M

Dept. of Endocrinology, Mater Misercordiae University Hospital, Dublin

\section{$\mathrm{DML} / \mathrm{UCD}$}

Hypertriglyceridemia is defined as a fasting serum triglyceride level of $>1.7 \mathrm{mmol} / 1$. Aetiology is multifactorial and it is a disorder associated with multiple complications. This case concerns a lady with hypertriglyceridemia who developed diabetes mellitus (DM) secondary to chronic pancreatitis.

Mrs C is a 34-year-old lady from Zimbabwe with severe hypertriglyceridemia who presented to the MMUH ED reporting a 3-week history of polydipsia, polyuria and abdominal pain. She is a known diabetic, secondary to chronic pancreatitis with eight admissions to MMUH in the previous two years. The patient was diagnosed with pancreatogenic DM nine months previously and was commenced on Metformin but discontinued the medications as she was experiencing intolerable side effects. Her HbAlc was 110 on admission with triglyceride levels of 29 , a cholesterol of 1.4 while HDL, LDL and non-HDL levels were unmeasurable. Her past medical history was significant for HIV for which she took antiretroviral therapy. She had a significant family history as both her father and sister were insulin dependent diabetics who experienced similar episodes of recurrent pancreatitis. Examination revealed mild epigastric tenderness with no evidence of tendon xanthoma, acanthosis nigricans, arcus senilis. Non-pharmacological management played an integral role in both hypertriglyceridemia and DM, focusing on a low-fat diet with weight loss and exercise. The patient attended a lipid clinic in a tertiary centre for the management of her hypertriglyceridemia and was currently managed on fenofibrate and atorvastatin. Humulin M3 and diamicron were commenced on this admission for
DM management with a plan for review of her blood sugar levels 3 weeks post discharge.

This case highlights the complex nature of hypertriglyceridemia and the range of complications associated with this disease resulting in the necessity of a multifactorial approach to appropriately manage this condition.

\section{Case Report: Challenges In The Management Of Chronic Pulmonary Aspergillosis}

Hughes K, O’Brien S, McLaughlin A

Dept. of Respiratory Medicine, St James' Hospital, Dublin

\section{DSE/TCD}

Introduction: Chronic Pulmonary Aspergillosis (CPA) affects approximately 3 million people worldwide ${ }^{1}$. The standard first line treatment is with azole antifungals ${ }^{2}$.

Case presentation: We report the case of a 43 -year-old male who presented with an episode of life-threatening massive haemoptysis on a background of known Chronic Pulmonary Aspergillosis. The patient had a long standing left upper lobe aspergilloma and bronchiectasis secondary to childhood Pertussis. While receiving treatment with azole antifungals the patient experienced adrenal suppression, which may have been the result of steroid potentiation or as a direct complication of azoles. This necessitated the cessation of azole antifungal therapy.

The patient then presented with what was his second episode of massive hemoptysis. Patient was treated with caspofungin under guidance by microbiology however this was felt to be suboptimal ${ }^{3}$. Interventional radiology could not attempt embolisation due to torturous vessels and risk of anterior spinal artery syndrome. The patient was reviewed by surgeons who subsequently performed a successful left sided pneumonectomy (the entire left lung had to be removed due to adhesions).

Discussion: This case highlights the challenges faced in the management of this condition. Adverse effects are common with azole antifungal medications and treatment options thereafter are often limited. This patient was faced with the difficult decision of choosing between the risk of massive haemoptysis in the future and major cardiothoracic surgery. Newer treatment modalities, such as transbronchial removal of intracavitary aspergilloma ${ }^{4}$, have been described in case series and represent exciting new options in the management of CPA which may allow some patients to avoid surgery.

References:

Global and Multi-National Prevalence of Fungal Diseases-Estimate Precision Felix Bongomin, Sara Gago, Rita O. Oladele, David W. Denning. J Fungi (Basel) 2017 Dec; 3(4): 57. Published online 2017 Oct 18. doi: 10.3390/jof3040057. PMCID: PMC5753159

Chronic Cavitary and Fibrosing Pulmonary and Pleural Aspergillosis: Case Series, Proposed Nomenclature Change, and Review David W. Denning,1 Kostantinos Riniotis,1 Richard Dobrashian,2 and Helen Sambatakou1 1 School of Medicine, University of Manchester, and 2 Department of Radiology, North Manchester General Hospital, Manchester, United Kingdom

Diagnosis and management of Aspergillus diseases: executive summary of the 2017 ESCMID-ECMM-ERS guideline. Ullmann, A.J. et al. Clinical Microbiology and Infection, Volume 24, e1 - e38

\section{Alcohol And Dementia- What's The Harm?}

Kiely O, O’Malley G

Dept. of Geriatrics, Sligo University Hospital

\section{WNW/NUIG}

Introduction: Dementia, a common disease is becoming more prevalent amongst the elderly however its relationship with alcohol is still not fully 
understood. This continues to prove challenging for diagnosis and management as we saw in one particular case.

Case Presentation: An 83-year old woman presented initially at Geriatric OPD by GP referral for recent cognitive impairment. She scored a MOCA of 21/30 with no other issues. She came to our attention 2 months later in ED with general decline, reduced appetite and right hip pain for 2 weeks. She was unsure if she had fallen. Her physical exam and vital signs were normal. Her bloods showed a CRP 129 and deranged LFTs. During admission it became apparent that she drank 28 units of wine per week for a number of years. A MOCA scored her a $14 / 20$ with $0 / 5$ in Delayed Recall and $1 / 5$ in Visuospatial/Executive Functioning. Her CT brain was inconclusive. She was diagnosed with Alcohol Induced Hepatitis. She presented 1 month later with pseudogout and again for the third time in 6 months with a hip fracture. Discussion: MOCA scores seen in Alcohol-Related Dementia show weaknesses in Delayed Recall and Visuospatial/Executive Functioning as in this case. Despite alcohol abuse for many years, this issue wasn't mentioned by GP or family members when memory issues first arose. There is conflicting data published regarding alcohol and its contribution to dementia. Some studies show a moderate intake of alcohol in the elderly contributes to reduced risk of dementia compared with abstention however; others highlight the higher incidence of dementia in those who previously had episodes of binge drinking. The question is could any of these admissions been prevented had her alcohol abuse been tackled or the subject broached regarding its impact on the patient's memory?

\section{A Rare Presentation Of Steroid Induced Psychosis Leading To ICU Admission}

Duncan A, Martin A

Dept. of General Medicine, Beaumont Hospital, Dublin

\section{DNE/RCSI}

Background: Steroid induced psychosis is a well-reported and rare phenomenon. The purpose of this case is to highlight an unusual presentation and diagnostic difficulties encountered.

Method: We present the case of a 63-year-old lady who was admitted for observation post reaction to infliximab for IBD. The patient received IV Hydrocortisone. The past medical history is relevant also for depression. The patient had an episode of vasovagal syncope and dropped their GCS to three with no focalized neurological deficit and equal pupils.

CT brain reported no evidence of acute abnormality. Anesthetics decided the patient would need to be transferred to the ICU for intubation with no change in GCS.

Neurology was urgently consulted for query metabolic encephalopathy vs. non-convulsive status-epilepticus. It was noticed that the patient was now resisting eye opening and the pupils were rolled backwards. The GCS remained unchanged. An urgent MRA, EEG and LP were completed, all within normal parameters.

After 36 hours of ICU admission the patient regained consciousness spontaneously and self-extubated. At this point a psychiatry opinion was sought.

It was felt the differential was;

1: Steroid induced psychosis

2: Functional Disorder

2: Hypoactive delirium

Conclusion: Steroid induced psychosis remains a diagnostic dilemma. The literature suggests that the prevalence can be as high as $6 \%$ depending on dose and risk factors such as psychiatric illness [1,2]. This case illustrates an extremely rare presentation. It is a diagnosis of exclusion and the pathophysiology is poorly understood [2,3]. A key issue was trying to explain this to the patient and understand what role, functional disorder plays. A functional disorder is now considered a "rule-in" diagnosis, remaining a difficult diagnosis in medicine [4,5]. New recommendations in managing these patients have recently been released and help guide in improving transparency in diagnosis and communication with patients [4].
References:1: Janes, M. and Kuster, S. (2019). Steroid-induced psychosis. Baylor University Medical Center Proceedings, 32(4), pp.614-615.

2: Dubovsky, A. and Arvikar, S. (2012). The Neuropsychiatric Complications of Glucocorticoid Use: Steroid Psychosis Revisited. Psychosomatics, 53(2), pp.103-115.

3: American Psychiatric Association Diagnostic and Statistical Manual of Mental Disorders. 5th ed Washington, DC: American Psychiatric Publishing; 2013.4: Adams, C. and Anderson, J. (2018). You've made the diagnosis of functional neurological disorder: now what? Practical Neurology, 18(4), pp.323-330.5: Chafetz, M., Bauer, R. and Haley, P. (2019). The other face of illness-deception: Diagnostic criteria for factitious disorder with proposed standards for clinical practice and research. The Clinical Neuropsychologist, pp.1-23.

\section{Whispering TB: A Case Of Laryngeal Tuberculosis}

Farrell A, Alnafisee D, McLoughlin H

Respiratory Dept., Portiuncula Hospital, Ballinasloe, Galway

\section{WNW/NUIG}

Background: Laryngeal TB represents less than 2\% of extra-pulmonary TB cases and is highly contagious ${ }^{1}$. Risk factors include smoking, alcohol, immunosuppression, immigrating from high-risk areas, and the emergence of multi-drug-resistant organisms ${ }^{2,3}$. Previously patients noticed odynophagia, weight loss and night sweats, recently the most common complaint is hoarseness ${ }^{3}$. There are two ways laryngeal TB can develop - by direct spread of bacilli in bronchial secretions or haematogenous spread from a distant primary focus ${ }^{1}$.

Case: A 30-year old woman presented to the Respiratory Department with a 3-week history of dry cough, dyspnea on exertion and intermittent low-grade fever on a background of a 5-month history of hoarseness. This had been investigated by Otolaryngologists and she was diagnosed with Idiopathic Laryngitis. Tuberculosis screening two years prior was negative.

Methods \& Results: Her evaluation consisted of: chest radiograph, HRCT, sputum culture, biopsy, Mantoux test, CT neck and routine blood tests, HIV and Hepatitis Screening.

A patient with no significant past medical or smoking history, chest $\mathrm{x}$-ray revealed hyperinflation and extensive bilateral pulmonary nodular densities particularly in the left peri-hilar region. Routine bloods and viral screens were normal.

HRCT results-multiple calcified nodules, characteristic tree-in-bud type opacification and bronchoscopy confirmed diagnosis of pulmonary TB. Endoscopy showed white plaques present bilaterally on the vocal cords. Previous histopathology showed inflamed granulation tissue and bronchoalveolar lavage was positive for AFB.

TB treatment was commenced, however there was some residual hoarseness due to the development of an anterior glottic web.

Conclusion: Patients are more likely to present to ENT surgeons because of the initial symptom of hoarseness, multiple tests must be completed before out-ruling TB. HRCT or sputum culture is recommended as TB may not be evident on initial xray. A collaborative approach between Respiratory and ENT team was evident throughout this case and will continue during the patient's prolonged course of treatment.

References:

1.Reis, J., Reis, C., Costa, D., Lucena, M., Schubach, A., Oliveira, R., Rolla, V., Conceição-Silva, F.and Valete-Rosalino, C. (2016). Factors Associated with Clinical and Topographical Features of Laryngeal, Tuberculosis. PLOS ONE, 11(4), p.e0153450.

2..Kandiloros, D., Nikolopoulos, T., Ferekidis, E., Tsangaroulakis, A., Yiotakis, J., Davilis, D. and Adamopoulos, G. (1997). Laryngeal tuberculosis at the end of the 20th century. The Journal of Laryngology \& Otology, 111(7), pp.619-621.

3 . Ling, L., Zhou, S. and Wang, S. (2010). Changing trends in the clinical features of laryngeal tuberculosis: a report of 19 cases. International 
Journal of Infectious Diseases, 14(3), pp.e230-e235.

Cardiovascular Risk Factors In Chronic Inflammatory Arthritides

Sheridan $\mathrm{R}^{1,2}$, Whelan $\mathrm{B}^{2}$, Silke $\mathrm{C}^{2}$, O'Sullivan $\mathrm{M}^{2}$

Dept. of Rheumatology ${ }^{1}$, Sligo University Hospital \& National University of Ireland, Galway; North Western Rheumatology Unit ${ }^{2}$, OLHM3, Co. Leitrim

\section{WNW/NUIG}

Background: Inflammatory arthritides (IA), including rheumatoid arthritis (RA), psoriatic arthritis (PsA) and ankylosing spondylitis (AS), are common in Ireland and impart a significant burden on both healthcare and quality of life. Patients with IA are at significantly increased risk of cardiovascular disease (CVD) due to a state of chronic inflammation and accelerated atherosclerosis, as well as traditional cardiovascular risk factors (TCVRFs).

It has been demonstrated that the relative risk is as high as 1.5 when compared to the general population, and similar in risk to diabetes. ${ }^{[1-3]}$

Aims: To investigate the prevalence of TCVRFs in IA patients.

Methods: Cross-sectional study of 318 patients with physiciandiagnosed IA who attended the outpatient's department in OLHM.

Results: Of our group of IA patients, 208 (65\%) had RA, 74 (23\%) had PsA and $36(11 \%)$ had AS. The mean age of all participants was 58.97 years (SD 14.86). 205 (64\%) were female.

Modifiable risk factors examined were the presence of hypertension, hypercholesterolemia, diabetes and active smoking. In our cohort, 191 $(60 \%)$ patients had at least one modifiable risk factor for cardiovascular disease. $111(35 \%)$ of patient's had just one risk factor, 64 (20\%) had two, $16(5 \%)$ had three and none had all four risk factors studied.

The most common risk factors were hypertension, (116 patients, 36\%) followed by hypercholesterolemia (96, 30\%) and smoking (63, 20\%). 21 $(7 \%)$ of patients were diabetic.

The incidence of serious cardiovascular disease in our patient cohort was high, with $30(9 \%)$ having a history of a significant cardiovascular event, defined as previous myocardial infarction or cerebrovascular accident, or having had previous coronary revascularisation.

Conclusion: The prevalence of TCVRFs remains high in our patient cohort. Recognition of cardiovascular risk factors is crucial in IA patients as they provide a readily treatable approach to reducing the otherwise high CVD burden. References: Aviña-Zubieta JA, et al. "Risk of cardiovascular mortality in patients with rheumatoid arthritis: a meta-analysis of observational studies." Arthritis Rheum 2008; 59:1690.

van Halm VP, et al. "Rheumatoid arthritis versus diabetes as a risk factor for cardiovascular disease: a cross-sectional study, the CARRE Investigation." Annals of Rheumatic Diseases 2009;68:1395-400.

Agca R, Heslinga SC, et al "EULAR recommendations for cardiovascular disease risk management in patients with rheumatoid arthritis and other forms of inflammatory joint disorders: 2015/2016 update." Annals of the Rheumatic Diseases 2017;76:17-28.

\section{My Feelings Form - Giving Children A Voice Through Colour And Cartoon}

Glancy C, Mulligan A, Gadencheva V, Sresthaporn N

Dept. of Child and Adolescent Psychiatry, University College Dublin

UCD School of Medicine, University College Dublin ${ }^{1}$, Dublin North City \& County Child and Adolescent Mental Health Service, Health Services Executive $^{2}$, Dept. of Child and Adolescent Psychiatry, University College Dublin $^{3}$, Dept. of Child Art Psychotherapy, University College Dublin ${ }^{4}$

\section{$\mathrm{DML} / \mathrm{UCD}$}

Background: The "My Feelings Form" (MFF) is a new self-report form designed by our group to measure emotional wellbeing/distress in children aged 4-13, using colour, pictures and emoticons, with a Likert scale. A validation study of the MFF in 314 children from the general population has been described (Sresthaporn N. et al., 2019).

Aims: To validate the MFF in the clinical population and to compare results in the clinic group with results of the MFF in the general population. Methods: Ethics approval was obtained from UCD and HSE. Children attending a Child and Adolescent Mental Health Service (CAMHS) were invited to complete the MFF, with parental consent. The MFF has a total possible score of 56, with 0 indicating no problems and 56 indicating maximum measured problems. An initial clinic sample of 14 patients is described; data collection is ongoing. The data was recorded into SPSS for analysis.

Results: The mean MFF score of the initial clinical group sample was 19.86 with a range of 5-50. This compares with a mean score of 16.08 in the general population, with a range of $4-38.35 \%$ of children in the initial clinical group reported to have "hurt themselves on purpose", compared with $23 \%$ of children in the school group. Furthermore, in both groups there was a significant negative correlation between "hurting themselves on purpose" and being able "to tell someone".

Conclusions: The MFF appears to be a user-friendly tool which may empower children in communicating their feelings. Results of measured problems were higher in the initial clinical group, yet both groups had negative responses recorded. Further research, which is currently underway, is evaluating a larger sample of CAMHS patients.

\section{A Case Of Anaemia Post Penetrating Stab Wound}

Allen J, McLornan L

Urology Dept., Beaumont Hospital, Dublin

\section{DNE/RCSI}

Introduction: The lumbar arteries are an uncommon site for injury following trauma. Pseudoaneurysm may result from blunt or penetrating trauma but always require a high index of suspicion for an early diagnosis. The initial injury can be missed and subsequently present later as a pseudoaneurysm. Here, we present an unusual case of penetrating injury to the posterior abdominal wall, where the injury to the lumbar artery lead to the development of a pseudoaneuysm and a subsequent anaemia.

Case Summary: A 24-year-old male of African origin, presented to the ED of Beaumont Hospital with ongoing lower back pain after suffering a penetrating stab wound in the right renal angle one week previously. Upon initial evaluation, he was noted to be hemodynamically stable. The patient had been assessed directly after the event but had discharged against medical advice before any treatment could be administered. No significant past medical history or family history were noted. Imaging on admission [CT AP] revealed an enhancing $2.3 \times 1.5 \mathrm{~cm}$ focus between the erector spina muscle and the psoas muscle likely centred on lumborum, representing a likely pseudoaneurysm. In conjunction with Vascular and IR, Ultra-Sound guided percutaneous injection of thrombin into the pseudoaneurysm was the most viable treatment option.

A total of three separate US thrombin injections were performed with the final repeat ultrasound revealing the previous pseudoaneurysm now sized at $1.3 \times 0.7 \mathrm{~cm}$, with no internal flow demonstrated. Satisfactory occlusion was achieved.

Serial FBC's showed a hypochromic microcytic anaemia despite no obvious source for bleeding on imaging. Iron/transferrin levels were within normal limits, folate levels were markedly decreased $[<2.0]$, sickle cell screen was normal, while a blood film was suggestive of a haemoglobinopathy. Our patient was commenced on folic acid supplementation and referred to the outpatient department with haematology for further follow up and testing to confirm a suspected thalassemia diagnosis.

An Unusual Case Of A Bile Leak Presenting 7 Weeks PostLaparoscopic Cholecystectomy

Leeson H, Donohoe C 
Dept. of Upper Gastrointestinal Surgery, St James's Hospital, Dublin

DSE/TCD

Introduction: Laparoscopic cholecystectomy is a common surgical procedure, one recognised complication is a bile leak, which is generally considered an early complication postoperatively. Bile leaks may be serious, potentially causing biliary sepsis and even death.

Case: A 33-year old woman presented to the emergency department complaining of severe abdominal pain, vomiting and diarrhoea, with abdominal tenderness having received an elective laparoscopic sleeve gastrectomy and cholecystectomy 7 weeks previously in Turkey. A computed tomography scan revealed free intra-peritoneal fluid around the gallbladder fossa and duodenum. The late timing and normal liver function tests made a bile leak seem unlikely. MRI liver/MRCP demonstrated the free fluid was resolving and she was discharged.

Five weeks later, the patient re-presented, again complaining of severe abdominal pain. The differential included a complication related to her sleeve gastrectomy such as herniation or necrosis. An interval increase in free fluid was observed on CT. Our differential evolved to include a bile leak, inflammation of a remnant cystic duct or a severe duodenitis. An unremarkable oesophagogastroduodenoscopy was followed by an endoscopic ultrasound which revealed inflammatory changes in the gallbladder fossa and free fluid. A bile leak was diagnosed.

The patient deteriorated, another abdominal CT demonstrated increasing free fluid and a MRI liver/MRCP highlighted a collection at the liver. The patient required biliary stenting and drainage of the liver collection.

Conclusion: While a bile leak in the immediate post-operative period is not uncommon, there are few reports of bile leaks presenting so late as the aforementioned case. A small leak may have been ongoing since the time of operation which became symptomatic at 7 weeks. This case highlights the need for a high index of suspicion in a patient re-presenting with acute abdominal pain at any point post-laparoscopic cholecystectomy where no definitive cause is identified despite multiple investigations.

\section{A Rare Case Of Peristomal Varices Causing Stomal Bleeding}

Hasnol MH, Caldwell M

Dept. of Surgery, Sligo University Hospital

\section{WNW/NUIG}

Background: Ectopic varices are a rare cause of gastrointestinal bleeding with associated portal hypertension, accounting up to only $5 \%$ of all variceal-related bleeding ${ }^{1}$. It is reported that $26 \%$ of them are from peristomal varices ${ }^{1}$ and they can present with potentially fatal unstable stomal bleed.

Case: We present a case of a 54-year-old lady with a 1-day history of unstable stomal bleeding on a background of subtotal colectomy with ileostomy due to refractory Crohn's colitis with associated primary sclerosing cholangitis. She had changed 3 stoma bags containing fresh blood and continued to have clotted fresh blood on admission. There was associated light-headedness and palpitations with her shock index of 1.6: heart rate $145 \mathrm{bpm}$ and blood pressure $87 / 65 \mathrm{mmHg}$. Her liver function test was deranged and her haemoglobin was $7.9 \mathrm{~g} / \mathrm{dl}$.

She was resuscitated immediately with urgent gastroscopy performed with no cause of bleeding found. Further investigation with CT abdomen and pelvis identified esophageal varices and later on multi disciplinary meeting found peristomal varices. She was managed conservatively initially having received 6 units of red cell concentrate and terlipressin. Following this, she underwent variceal embolisation under radiological imaging to which was effective in achieving haemostasis as an inpatient. Her follow up plan was to undergo TIPS procedure for the portal hypertension.

Conclusion: In conclusion, the rare presentation of stomal haemorrhage with suspected or diagnosed chronic liver disease should warrant clinicians high suspicion of index for peristomal varices. With early detection, both the appropriate acute and chronic management, to stop the active bleeding and to treat the portal hypertension can be achieved promptly. In spite there are various therapeutic management described in the literature, there are no set guidelines on managing stomal bleeding or ectopic variceal bleeding. Regardless, a multi-disciplinary approach for these patients is paramount in their care.

Reference:

1) Norton I, Andrews J, Kamath P. Management of ectopic varices. Hepatology 1998; 4: 1154-1158

\section{A Novel Case Of Rouxxxx-En-Y Gastric Bypass}

McMurray M, Naqeeb J, Sabahi J, Bodnar Z

Dept. of Surgery, Letterkenny University Hospital

\section{WNW/NUIG}

Aims and Objectives: Tetrasomy X (48, XXXX) is an incredibly rare chromosomal disorder caused by the presence of four $\mathrm{X}$ chromosomes, rather than the expected one or two. The disease was first described in 1961 , in that time there have been approximately 100 cases reported worldwide, however, given poor access to genetic screening and various phenotypes inhibiting confirmation, only 60 cases have been described in the medical literature.

Given the lack of knowledge surrounding patients with Tetrasomy X, medical professionals may be reluctant to intervene as they would otherwise. As a result, we have decided to present this case of Roux-en-Y Gastric Bypass in a morbidly obese tetrasomic patient to display not only the effectiveness, but the safety in performing this procedure in not just Tetrasomic patients, but the entire morbidly obese demographic.

Methods: The 46-year-old woman was referred to our Metabolic Surgery Unit for further management from Galway University Hospital in 2017. Since 2008, she was seen by multiple specialists, in various fields, all involving a variety of different diet and weight loss programs to no avail. Due to her significant comorbidities and her genetic disorder, the relative risk of surgery was previously deemed too high to be a viable option in her case.

She was seen in our clinic with a BMI: $48(148 \mathrm{~kg})$ and symptomatic choleliathiasis, for which she was attending the accident and emergency department on a weekly basis. It was decided that she was a safe candidate for Laproscopic Roux-en-y Gastric Bypass and cholecystectomy as one procedure and on March $11^{\text {th }}, 2019$ the team proceeded with surgery.

Results: The patient made an excellent recovery post-op, suffering no complications and enjoying a significant improvement in her quality of life. Her BMI 6-months post-op was $37.8(123 \mathrm{~kg})$, which equates to a total of $25 \mathrm{~kg}$ lost. As a result of this weight loss, her related comorbidities (joint pain, leg ulcers, metabolic syndrome) have already begun to improve. This was seen not only clinically, but also in the reduction of her diabetic and antihypertensive medication. In addition to the weight loss, she no longer suffered from any gallbladder related complaints and self reported increases in mood and well-being.

Discussion: Bariatric surgery has consistently been demonstrated as a safe and effective intervention for morbid obesity, with results superior to any non-surgical intervention. Despite greater body weight loss, higher rates of remission of type II diabetes mellitus and metabolic syndrome, superior reductions in the use of antigylcaemics, antihypertensive, and lipid lower drugs, Ireland still lags behind most developed nations in its use of metabolic surgery, regardless of the fact obesity is a significant public health issue.

Our case, the first ever Roux-en-Y Gastric Bypass procedure performed in a patient with the tetrasomy $\mathrm{X}$ chromosomal abnormality, is unique to the bariatric literature and an excellent example to highlight the importance and safety of bariatric surgery as a whole.

Medication Safety: An Audit Of Kardex Transcription Discrepancies

Rathakrishnan S, Tan LY, English M, Nisar A, Slattery N 
University of Limerick Hospital Group, Limerick

MW/UL

Introduction: Medication errors (ME) can lead to serious consequences; from increased hospital length of stay (LOS), to death ${ }^{1}$. Transcription error is a specific type of ME that is borne from erroneous data entry ${ }^{2}$. The objective of this audit is to examine the prevalence of transcription error in University Hospital Limerick (UHL), a university teaching hospital, by inspecting the paper-based Kardex system being utilised.

Methods: A randomised list of inpatients, whose LOS extended beyond 14 days, and thus required more than 1 Kardex, was generated. All inpatient Kardexes on current admission were analysed for transcription errors. From 35 inpatients, 85 Kardexes were derived and studied retrospectively. Two types of transcription errors were identified; critical errors (CE) and non-critical errors (NCE). CE include omitted drugs, or discrepancies in dose, frequency, route, timing of time-sensitive drugs, or a combination of these. NCE include omission of prescriber identification and incorrect start dates amongst other factors.

Additional data was gathered on Kardex re-write checks led by pharmacists, to analyse their effect on transcription error rate. CE were communicated to the respective teams.

Results: 51 out of the 85 Kardexes examined were transcribed from a previous Kardex. 115 transcription errors were derived from the 51 rewritten Kardexes.

Each transcribed Kardex contained an average of 2.3 discrepancies. 7.8\% consisted of erroneous omissions. CE and NCE consisted of $20 \%$ and $80 \%$ of the discrepancies, respectively.

$50.98 \%$ of re-written Kardexes were formally reviewed by pharmacists. Errors in checked and unchecked Kardexes were $53.85 \%$ and $60 \%$ respectively.

Conclusion: As evidenced by this audit, there is a rate of 3.29 errors/ patient in transcribing Kardexes.

We propose (i) the adoption of an electronic prescribing system, removing the need for transcribing drugs every 14 days, thereby reducing the rate of transcription-related errors; and (ii) increments in the number of checks for re-written Kardexes.

References:

Allan E, Barker K. Fundamentals of medication error research. Am J Hosp Pharm 1990;47:555-71.

Ringold DJ, Santell JP, Schneider PJ, Arenberg S. ASHP national survey of pharmacy practice in acute care settings: prescribing and transcribing1998. Am J Health Syst Pharm 1999;56:142-57.

\section{A Case Of Perforated Appendicitis: Unusual Presentation Of Crohn's Disease}

\section{Karlsson R, Donohoe C}

Dept. of Surgery, St. James's Hospital, James’s St., Dublin

\section{DSE/TCD}

Introduction: The first presentation of Crohn's Disease can mimic the presentation of numerous surgical pathologies. This may be difficult to distinguish clinically while managing a patient in the acute setting.

Case Presentation: A 16-year old male presented asymptomatically to the Emergency Department following complete resolution of five days of right iliac fossa pain associated with dysuria and non-bloody, non-mucous diarrhoea. Pyrexia of $38.2^{\circ} \mathrm{C}$ was measured. Collateral history confirmed no family history of autoimmune or inflammatory disease and the patient denied any previous episodes of altered bowel habit. Examination revealed a soft, non-tender abdomen with a palpable mass in the right iliac fossa. There were no extra-intestinal features of inflammatory bowel disease.

Serological investigation revealed raised inflammatory markers; a white cell count of 14.1 and CRP of 211. Imaging revealed perforated appendicitis with multiple abscesses, as well as mural thickening of the terminal ileum. Following MRI analysis, the small bowel changes were felt to be reactive to the multiple abscesses, and while a differential diagnosis of inflammatory bowel disease was considered at this stage, it was felt to be less likely than primary appendiceal inflammation.

The patient was managed conservatively with intravenous antibiotics, and was discharged once inflammatory markers had returned to within normal limits. The patient remained asymptomatic throughout his entire inpatient stay.

Follow-up colonoscopy revealed a distorted ileocaecal valve with active ulceration and inflammation of the bowel wall, consistent with Crohn's Disease. Biopsies have been taken and the patient will be seen in a gastroenterology clinic for further assessment.

Conclusion: This case serves as a reminder as to the importance of formulating a differential diagnosis, even when faced with a seemingly archetypal presentation. The recently published results from the RIFT Study Group ${ }^{3}$ further highlight the importance of imaging in the work-up of these patients. Reference:

${ }^{3}$ (2019), Evaluation of appendicitis risk prediction models in adults with suspected appendicitis. Br J Surg. doi:https://doi.org/10.1002/bjs.11440

Cardiac Metastasis Of Lung Cancer In A 56-Year-Old Male

Kavanagh A, Daly A, Young VK

Dept. of Cardio-thoracic Surgery, St James's Hospital, Dublin

DSE/TCD

Introduction: Cardiac tumours are rare. The most common primary cardiac tumour is atrial myxoma, however, cardiac metastasis is reported to be 20 to 40 times more common (1). Surgical intervention is rarely indicated and has a limited role in the setting of cardiac metastasis.

Case Presentation: A 56-year-old male presented with a two-month history of persistent dyspnoea on exertion, fatigue and weight loss. He had a significant background history of non-small cell lung cancer (NSCLC) (pT4N0M0 R0) which was treated with a left upper lobectomy eight months prior and subsequently adjuvant chemotherapy. A computed tomography thorax on admission demonstrated a new right atrial mass extending into the inferior vena cava. Further imaging with transoesophageal echocardiogram and cardiac magnetic resonance imaging helped characterize the anatomy of the right atrial mass with appearance favouring tumour versus thrombus. Positron emission tomographycomputed tomography showed no evidence of distant metastasis and a biopsy of the mass under interventional cardiology was attempted but was deemed inconclusive. Given the evidence of obstruction the right atrial mass was resected despite the presence of stage IV disease. Surgical technique is discussed. Ultimately, histology confirmed NSCLC, compatible with previous resection and in keeping with metastatic lung cancer.

Discussion: Although considered a rare site for tumour metastasis the incidence of cardiac metastasis is reported up to $18 \%$ in literature with lung cancer accounting for approximately $30-40 \%$ of cases described (2). Cardiac metastasis is often silent and surgical intervention is rarely required. However, as evident from this case surgery may be warranted in the event of haemodynamically-threatening features. This case highlights the importance of accurate diagnosis and characterization of a cardiac mass, the indications for surgery and the surgical technique used.

\section{References:}

Kassop D, Donovan M, Cheezum M, Nguyen B, Gambill N, Blankstein $\mathrm{R}$ et al. Cardiac Masses on Cardiac CT: A Review. Current Cardiovascular Imaging Reports. 2014;7(8).

Verma, V., Talmon, G. and Zhen, W. (2015). Intracardiac Metastasis From Non-Small Cell Lung Cancer. Frontiers in Oncology, 5.

\section{Caught Flat Footed - Peripheral Neuropathy Due To Immune Complex Vasculitis}

Doris $\mathrm{M}^{1}$, O’Sullivan $\mathrm{F}^{2}$ 
Emergency Dept. ${ }^{1}$; Geriatrics/Internal Medicine ${ }^{2}$, Sligo University Hospital, Sligo

\section{WNW/NUIG}

Background: We present the case of a 75-year old gentleman presenting to Sligo Emergency Department with a 5 day history of painful right foot drop and a 1 day history of painful left foot drop, on a background of coeliac disease and resected oesophageal cancer in-situ.

On examination he had bilaterally absent dorsiflexion and proprioception loss at the hallux, present at ankle. A purpuric rash was noted on the right ankle. Inflammatory markers were elevated with CRP 117 and ESR 62. He was admitted on a working diagnosis of vasculitis.

Further testing showed an elevated rheumatoid factor $>600$, negative anticcp, low $\mathrm{C} 4$ and normal C3. A skin biopsy showed immune complex deposition. The broad diagnosis therefore is of an immune complex small-vessel vasculitis leading to mononeuritis multiplex. The patient was commenced on 5 days of IV methylprednisolone to good effect, with resolution of the left foot and improvement in the right.

Discussion: Vasculitis is rare in Ireland with an incidence of $15 /$ million $^{1}$, however an important one to be aware of as prompt treatment can save life and limb.

Small-vessel vasculitides have a tendency to cause peripheral neuropathy $^{2}$, particularly in the lower limbs. This particular case suggested a cryoglobuulinaemia due to the presentation, and the presence of high rheumatoid factor and low C4. However, the cryoglobulin assay has not produced a typical pattern of precipitation.

A high index of suspicion should be maintained in anyone with an acute onset peripheral neuropathy, especially with raised inflammatory markers or visible ulceration. Specific testing to find the exact subtype can be challenging and lengthy as in this case, however more important is the broad diagnosis, which can largely be done clinically with a thorough history and exam, and initial treatment which is generally high dose glucocorticoids ${ }^{3}$.

\section{References:}

Molloy E, Clarkson M, Griffin M, Little M, Power J. "VINE": The Vasculitis Irish Network - A summary proposal for inclusion in a European Reference Network. Vasculitis Ireland Network. 2019.

Moore, P. (2000). Vasculitic neuropathies. Journal of Neurology, Neurosurgery \& Psychiatry, 68(3), pp.271-274.

Blaes F. Diagnosis and therapeutic options for peripheral vasculitic neuropathy. Therapeutic Advances in Musculoskeletal Disease. 2015;7(2):45-55.

\section{Idiopathic Pulmonary Arterial Hypertension?}

Rigney K, O’Brien E

Respiratory Dept. Beaumont Hospital, Dublin

\section{DNE/RCSI}

Introduction: Pulmonary arterial hypertension $(\mathrm{PAH})$ is a rare condition defined as a MPAP of $\geq 25 \mathrm{mmHg}$ at right heart catheterisation. Estimated prevalence $10-52$ cases per million ${ }^{1}$. PAH is subcategorised according to underlying pathogenesis, including idiopathic, heritable, drug/toxin-induced and PAH associated with other conditions (connective tissue diseases, HIV, congenital heart disease and schistosomiasis) ${ }^{2}$. Accurate diagnosis is crucial as the management is different for each subcategory.

Case Description: A 35-year-old female, EP presented to the emergency department with a week-long history of progressive exertional dyspnoea and palpitations. No significant medical history, non-smoker, normal BMI. Recently stopped COCP. Family history positive for PE. Tachycardic, otherwise vitally stable. Clinical examination revealed loud P2. ECG showed RV strain. Chest X-ray showed evidence of dilated pulmonary outflow tract. Routine bloods remarkable for a transaminitis and raised BNP, pregnancy test negative. Commenced on therapeutic anticoagulation. Echocardiogram demonstrated a dilated RV and positive 60/60 sign. CTPA negative for PE, but demonstrated dilated main PA measuring $3.6 \mathrm{~cm}$, in keeping with $\mathrm{PAH}^{3}$. Anticoagulation stopped following CTPA. MPAP at RHC $60 \mathrm{mmHg}$, diagnostic of $\mathrm{PAH}^{4}$.V/Q scan showed no evidence of chronic thromboembolic disease. Subsequent investigations revealed positive $\mathrm{HCV}$-antigen but negative $\mathrm{HCV}$-antibody. HCV-PCR showed undetectable viral load. No risk factors for HCV. HIV negative. Furthermore, her IgG was raised, with anti-smooth muscle antibody, ANA, Ro-52, RF, anti-CCP and ANCA positive. Rheumatology and hepatology consulted. US liver unremarkable, no evidence of CTD on clinical examination. Discharged with follow up arranged at MMUH PAH clinic, rheumatology and hepatology.

Discussion: There has been no reported correlation between HCV infection and PAH however there have been several cases of PAH associated with autoimmune hepatis, ANCA-associated vasculitis and Sjögren's syndrome ${ }^{5-8}$. The significance of these positive results for EP is unknown at present. Conclusion: This case demonstrates a rare clinical condition and the complexities related to diagnosis.

References:

Hoeper M, Simon R. Gibbs J. The changing landscape of pulmonary arterial hypertension and implications for patient care. European Respiratory Review. 2014;23(134):450-457.

Simonneau G, Gatzoulis M, Adatia I, Celermajer D, Denton C, Ghofrani A et al. Updated Clinical Classification of Pulmonary Hypertension. Journal of the American College of Cardiology. 2013;62(25):D34-D41.

Edwards P, Bull R, Coulden R. CT measurement of main pulmonary artery diameter. The British Journal of Radiology. 1998;71(850):10181020.

Kiely D, Elliot C, Sabroe I, Condliffe R. Pulmonary hypertension: diagnosis and management. BMJ. 2013;346(apr16 1):f2028-f2028.

Kaneko F, Yokomori H, Tahara K, Takeshita T, Takeuchi H, Yoshida H et al. Autoimmune Hepatitis Associated with Pulmonary Arterial Hypertension. Internal Medicine. 2008;47(22):1971-1976.

Naito A, Terada J, Tanabe N, Sugiura T, Sakao S, Kanda T et al. Autoimmune Hepatitis in a Patient with Pulmonary Arterial Hypertension Treated with Endothelin Receptor Antagonists. Internal Medicine. 2014;53(7):771-775.

Launay D, Hachulla E, Hatron P, Jais X, Simonneau G, Humbert M. Pulmonary Arterial Hypertension: A Rare Complication of Primary Sjögren Syndrome. Medicine. 2007;86(5):299-315.

Launay D, Souza R, Guillevin L, Hachulla E, Pouchot J, Simonneau G et al. Pulmonary arterial hypertension in ANCA-associated vasculitis. Sarcoidosis Vasc Diffuse Lung Dis. 2019;2006 Oct;23(3):223-8.

\section{Delivery Discussions In University Maternity Hospital Limerick}

Doheny J, McKeown C, Imcha M

Dept. of Obstetrics, University Maternity Hospital Limerick

$\mathrm{MW} / \mathrm{UL}$

Background: University Maternity Hospital Limerick is a tertiary care centre with approximately 4500 births annually. The centre is committed to the emotional wellbeing of all patients, evidenced by the introduction of a dedicated perinatal mental health team in July 2018.

Aim: This study aimed to explore patient's perspective surrounding the events and communication at the time of delivery.

Methods: A questionnaire was administered in person to all patients who delivered within a 7-week period, beginning in August 2019. Some elements of the questionnaire included the mode of delivery, if any complications occurred and whether the patient felt their concerns were addressed. Patients were also encouraged to make comments in relation to any concerns that may have arisen.

Results: In total 100 patients were included. 45 women had a spontaneous vaginal delivery, 19 required instrumental deliveries (vacuum/forceps) and the remainder underwent caesarean section. 7 patients 
experienced a complication at the time of delivery and all felt as though their concerns were adequately addressed. Comments were provided by 56 patients.

Discussion: A delivery discussion provided a platform for new mothers to inform us of any concerns they may have had. The majority of patients felt their concerns were addressed and thankfully many positive comments were provided. In addition to this, having the opportunity to discuss their delivery has acted as a secondary debrief.

Conclusion: Communication between clinical team members and patients remains instrumental in providing the level of care we strive to achieve.

\section{Rare ECG Warning Signs: A Case Report On Wellens Syndrome}

Healy R, Sheehy M

Dept. of Respiratory Medicine, Mullingar Midlands Regional Hospital, Co. Westmeath

\section{DML/UCD}

Introduction: Wellens Syndrome is a rare presentation of T wave abnormalities on electrocardiogram in patients presenting with unstable angina. These $\mathrm{T}$ wave changes are highly specific for critical stenosis of the left anterior descending coronary artery. The criteria include; characteristic $\mathrm{T}$ wave changes, a history of anginal chest pain, normal or minimally elevated cardiac enzyme levels, ECG without $Q$ waves, without significant ST-segment elevation and a normal precordial R-wave progression. There are two forms of T wave abnormalities that occur with Wellens syndrome. Type A $(25 \%)$ is a biphasic $\mathrm{T}$ wave with initial positivity and terminal negativity. Type B (75\%) is a deeply and symmetrically inverted T wave. The danger with Wellens syndrome is that patients can be pain free by the time of ECG and have normal cardiac enzymes but they are at an extremely high risk for MI within days or weeks.

Presentation: I report on a case of a 68-year-old lady with recently diagnosed COPD, re-presenting to ED with increasing SOB at rest, productive cough and central chest pain. Serial troponins were significant and ECG findings included TWI. The patient was discussed with cardiology who advised it was potentially a Wellens type picture. The lady was referred for angiogram. Furthermore, the patient successfully underwent PCI to her LAD.

Discussion: Wellens syndrome was first described in 1982. T wave inversion on ECG is a relatively common presentation and should always be carefully investigated. The accurate diagnosis of Wellens syndrome can be challenging to make in pain free patient but should always be considered as a potential cause if there are characteristic ECG changes.

Conclusion: The recognition of these subtle ECG changes is crucial to avoid anterior MI in the future for these patients and to prevent significant mortality and morbidity.

Foramen Magnum Stenosis In Achondroplasia - A Complication With Potentially Fatal Outcomes

Corley L, McDonnell C

Discipline of Paediatrics, Trinity College Dublin

\section{$\mathrm{DML} / \mathrm{UCD}$}

Background: Achondroplasia is the most common inherited bone dysplasia, with an incidence of approximately $1: 20,000$ live births ${ }^{(1)}$. It is caused by abnormal endochondral ossification of the skeleton due to gain-of-function mutations in the FGFR3 gene. Cervical medullary compression due to foramen magnum stenosis is estimated to affect $5-10 \%$ of patients with achondroplasia ${ }^{(1)}$. Compression of the medulla may result in quadriparesis, sleep apnoea or respiratory arrest.

Case Report: We present the case of L, a 12-week old girl with achondroplasia. L acutely stopped breathing while seated in a standard 90 degree car seat during a short car journey. Her brother noted that her head had dropped, a frequent occurrence. When her chin was lifted she was frankly cyanotic and in respiratory arrest. Her mother administered CPR for approximately 20 minutes until ambulance staff arrived. She required intubation, ventilation and emergency transfer to Temple Street. MRI Brain and C-Spine in conjunction with a neurosurgical assessment suggested cranio-cervical junction obstruction secondary to foramen magnum stenosis. L proceeded to surgical decompression. The procedure and post-operative course were uncomplicated with no further apnoeic episodes. $\mathrm{L}$ is progressing well developmentally and is meeting milestones appropriately. She is receiving full multidisciplinary input.

Discussion/ Learning Points: This case demonstrates the very real risks posed by foramen magnum stenosis to children with achondroplasia. Given the magnitude of morbidity associated with this issue, this case

highlights the importance of appropriate assessment, prompt identification and timely intervention in the prevention of potentially fatal outcomes. Consistent approach to optimal assessment, evaluation and management of foramen magnum stenosis is lacking ${ }^{(2)}$, although screening rates have improved since the publication of AAP surveillance guidelines.

(3) We advocate screening in symptomatic children while we await the findings of a new Pan European study ${ }^{(4)}$.

\section{References:}

1. Coi, A. and Santoro, M. (2019). Epidemiology of achondroplasia: A population - based

study in Europe. American Journal of Medical Genetics Part A .

2. White, K. and Bompadre, V. (2015). Best practices in the evaluation and treatment of

foramen magnum stenosis in achondroplasia during infancy. American Journal of

Medical Genetics Part A , 170(1), pp.42-51.

3. Nadel, J., Wilkinson, D. and Garton, H. (2019). Screening and surgery for foramen

magnum stenosis in children with achondroplasia: a large, national database

analysis. Journal of Neurosurgery: Pediatrics , 23(3), pp.374-380.

4. Cheung M, Alves, I, Hagenas L, Mohnike K (2019), Meeting report from the

achondroplasia foramen magnum workshop, Salzburg, Austria. Bone $127 ; 499-502$

\section{A Case Of Undiagnosed Factor X Deficiency Presenting As Severe Menorrhagia}

Madden Doyle L, Rajab H

Dept. of Gynaecology, Beaumont Hospital, Dublin

\section{DNE/RCSI}

Abnormal uterine bleeding (AUB) and heavy menstrual bleeding (HMB) are common - and often debilitating - problems, affecting 3-30\% of women in their reproductive years. The prevalence of coagulopathy in this patient demographic is however, often overlooked, particularly in the absence of other manifestations. Often, in primary care, a thorough workup is not undertaken and symptoms are managed purely from a gynaecological perspective. In this case, a 37-year old woman, P2+0, presented to ED with menorrhagia and symptomatic anaemia, requiring blood transfusion. Her past medical history was significant for recurrent iron deficiency anaemia over 15 years, requiring multiple iron transfusions. Additionally, she had a sleeve gastrectomy performed abroad, with associated $57 \mathrm{~kg}$ weight loss over the preceding 9 months. Laboratory results showed deranged coagulation screen, with prolonged PT of 1.5, while aPTT remained normal. Haematology were consulted, who advised undergoing factor II, V, VII \& X assays. Results of same showed low factor $\mathrm{X}$ assay at 0.29 . Haematinics were also performed, yielding a low B12 \& folate. As an inpatient, multiple blood transfusions, tranxaemic 
acid, vitamin K \& B12 and folate replacement were administered. On discharge, referrals were made to upper gastrointestinal surgery $\&$ to the national coagulation centre to undergo total abdominal hysterectomy \& bilateral salpingoophrectomy. PCC will be administered prophylactically pre-operatively. This case highlights the often multifactorial nature of menorrhagia. While an underlying coagulation disorder can be suggested on history, in this case there was no additional indicative symptoms. FIGO classification of AUB subdivides aetiology into structural and non- structural, the latter of which can often be overlooked. However, a specific cause for HMB is often not identified in up to $50 \%$ of cases. Research suggests a substantial number of these cases can be accounted for by mild undiagnosed bleeding disorders, and we suggest patients should be investigated for same.

Acute Presentation Of Temporal Lobe Abscess Secondary To Cholesteatoma: A Case Report

Macarulay $\mathrm{R}^{1}$, Merry $\mathrm{C}^{1}$, Lennon $\mathrm{P}^{2}$

Depts. of Infectious Diseases ${ }^{1}$, Otolaryngology ${ }^{2}$, St. James's Hospital Dublin

\section{DSE/TCD}

Introduction: Cholesteatomas are benign growths of keratinising squamous epithelium in the ear that can be congenital but are more commonly acquired. They are feared for their invasive and destructive growth potential if left untreated.

Case presentation: We present a case of a 67 -year old male brought in by ambulance with acute onset dysphasia, left facial droop, and right sided inattention. CT brain on admission showed no acute intracranial haemorrhage and he was thrombolysed. On collateral history, it was revealed the patient suffered from chronic otitis media dating back to childhood, and had previous right mastoidectomy. Of note he recently had prolonged infection managed with ciprofloxacin.

MRI brain post-thrombolysis confirmed initial CT findings of aggressive cholesteatoma in left middle ear cavity with breech of tegemen, extension into inferior left temporal lobe and early development of cerebral abscess. Neurosurgery was not indicated acutely due to small abscess size.

Under ENT, he underwent left cortical mastoidectomy, facial nerve decompression and grommet insertion with no peri-operative complications. Intra-operative swabs grew Pseudomonas and Ent Faecalis and IV meropenem and vancomycin were commenced.

A number of MRIs were performed post-operatively, and MRI day 4 post op showed interval increase in size. The patient was briefly transferred for neurosurgical opinion however intervention was ultimately not indicated due to size remaining static. He was transferred back for completion of 6 weeks of antibiotics. He improved clinically and MRIs showed progressing decrease in abscess size until eventual complete resolution.

Discussion: Cholesteatomas resulting in brain abscess are a rare but recognised complication ${ }^{1}$ with a reported mortality rate of $36 \% .^{2}$ This case highlights an unusual acute presentation with LMN pattern of left sided facial droop due to invasion of left facial nerve within the middle ear and dysphasia and right sided inattention due to left temporal lobe invasion.

\section{References:}

1. Duarte MJ, Kozin ED, Barshak MB, Reinshagen K, Knoll RM, Abdullah KG, et al. Otogenic Brain Abscesses: A Systematic Review. Laryngoscope Investigative Otolaryngology 2018. p. 198-208.

2. Samuel J, Fernandes CM, Steinberg JoL. Intracranial otogenic complications: a persisting problem. Laryngoscope 1986. p. 272-8.

Audit Of Venous Thromboembolism (VTE) Risk Assessment, Thromboprophylaxis Prescribing And Compliance In Surgical Admissions To Portiuncula University Hospital (PUH)

McMorrow M, McAuliffe D, Cashen A, Garvin J
Dept. of Surgery, Portiuncula University Hospital, Co. Galway

\section{WNW/NUIG}

Background: As per the HSE Quality Improvement Division's report "Preventing Blood Clots in Hospitals", venous thromboembolism (VTE) is associated with $9 \%$ of all deaths. "Optimum prevention of VTE requires risk assessment of every inpatient early after the decision to admit them to hospital". ${ }^{1}$

Aims: To assess whether surgical patients in PUH have a VTE risk assessment performed on admission, if thromboprophylaxis is prescribed through thromboembolic stockings (TEDs) and low molecular weight heparin (LMWH), and if TEDs are worn by patients if prescribed.

Methods: All surgical admissions over 7 days (28/11/19-04/12/19), except for paediatric patients, were included. The drug kardex of each patient was examined to assess whether a thromboprophylaxis risk assessment was undertaken. Further examination involved determining if TEDs, LMWH or both had been prescribed, and if not, was a reason documented. Finally, patients were examined to see if TEDs were being worn if prescribed. If the patient had been discharged by the time of assessment, nursing staff documentation of a TEDs check was recorded as worn; no documentation was recorded as N/A.

Results: There were 44 adult surgical admissions. 0 patients had a risk assessment carried out. 26 (59\%) were charted for both TEDs and LMWH. $11(25 \%)$ for TEDs alone, $2(18 \%)$ of which had reasoning documented. $7(16 \%)$ were charted for neither, 2 (29\%) of which had reasoning documented. Of the 37 patients charted for TEDs, 27 were in-patients with $5(19 \%)$ wearing TEDs on examination. Of the 10 discharged, only 1 had documentation of TEDs checked.

Conclusion: No patients were risk assessed for VTE and there was poor documentation. There was also poor adherence to the use of TEDs. Further education of admitting doctors and nursing staff of the importance of VTE risk assessment and prevention is essential. Furthermore, an investigation into other methods to increase VTE risk assessment is needed. Reference:

National Medication Safety Improvement Programme. Preventing Blood Clots in Hospitals. Kilkenny: Health Service Executive; 2018. 56 p.

\section{An Audit Of The Prescription Of Sleeping Tablets In The Elderly} Population In A Model 3 Hospital In The West Of Ireland

Garman T, Hembrecht S, Waldron R, Barry K, Khan I, Khan W Dept. of General Surgery, Mayo University Hospital, Co. Mayo

\section{WNW/NUIG}

Introduction: The use of sleeping tablets in hospitals, nursing homes and the community is increasing [1][2] despite well documented evidence detailing multiple risks to patients, in particular elderly populations. Adverse psychomotor events, falls and cognitive impairment [2] are commonly reported side effects, with sleeping tablets also contributing to the multifaceted issue of polypharmacy [1].

Aim: To assess current prescribing practices of sleeping tablets in Mayo University Hospital (MUH).

Method: Patient's Kardexes from all surgical and medical wards were audited for regular, as required or stat prescriptions of drugs commonly used as sleeping tablets over a one-week period. "Sleeping tablets" were defined as all $\mathrm{Z}$ hypnotics and benzodiazepines (specified for night-time prescription).

Results: 161 Kardexes were audited, with 39 patients prescribed a sleeping tablet (24.2\%). $87 \%$ of these patients were over the age of 65 , and $38.4 \%$ over the age of $80.79 .5 \%$ of medications used were $Z$ hypnotics (Zolpidem \& Zopiclone), and 20.5\% were benzodiazepines (Temazepam, Alprazolam, Lorazepam, Diazepam). 51.3\% were regular prescriptions, $30.7 \%$ as required and $18 \%$ stat prescriptions. 
Discussion: An overwhelming majority (87\%) of patients receiving sedative medications in MUH were over 65, despite current literature advising against this practice [3].

Over half of sleeping tablets prescribed were a regular prescription, indicating widespread use of sedative medication in the community. It has been proven that discontinuing benzodiazepines during hospital admissions reduces the use after discharge [2] thus we should prioritise stopping these medications where possible.

Conclusion: There is a demonstrable lack of awareness of the risks of prescribing sedative medications in the elderly and no clear consensus on appropriate prescribing practice in MUH at present. An education session will be arranged with the hospital pharmacy department with the hope to improve this awareness and the practice will be re-audited to assess for improvement. References:

1. Halvorsen, K., Selbaek, G. and Ruths, S. (2016). Trends in potentially inappropriate medication prescribing to nursing home patients: comparison of three cross-sectional studies. Pharmacoepidemiology and Drug Safety, 26(2), pp.192-200.

2. McMillan, J., Aitken, E. and Holroyd-Leduc, J. (2013). Management of insomnia and long-term use of sedative-hypnotic drugs in older patients. Canadian Medical Association Journal, 185(17), pp.1499-1505.

3. American Geriatrics Society 2019 Updated AGS Beers Criteria ${ }^{\circledR}$ for Potentially Inappropriate Medication Use in Older Adults. (2019). Journal of the American Geriatrics Society, 67(4), pp.674-694.

\section{Ensuring Co-Prescription Of Appropriate Gastro-Protective Agents In Elderly Patients Taking Daily Aspirin}

O' Callaghan MP', O' Callaghan $\mathrm{M}^{2}$, O' Callaghan $\mathrm{E}^{3}$, O' Callaghan Marcella ${ }^{3}$, O' Callaghan $\mathrm{M}^{3}$

Dept. of General Surgery ${ }^{1}$, Castlebar Hospital, Mayo University Hospital; Dept. of Orthopaedic Surgery ${ }^{2}$, University Hospital Limerick, Limerick City; Bruff Family General Practice ${ }^{3}$, Bruff, Co. Limerick

\section{$M W / U L$}

Background: Patients taking daily aspirin are at risk of both gastrointestinal (1) and intracranial haemorrhage. (2) Current NICE guidelines advise concurrent gastro-protective therapy in patients taking daily aspirin, particularly those aged over 70 years.

Aim: Our primary goal was to audit whether elderly patients taking aspirin were appropriately co-prescribed a proton pump inhibitor (PPI).

Method: The inclusion criteria for the audit were patients over the age of seventy who were prescribed daily aspirin. Patient charts were analysed to establish the current status of the patient (active patient, RIP, inactive e.g. moved practice) and if they were still taking aspirin. Data were gathered retrospectively using a Socrates data collection programme and results were compiled into an Excel sheet.

Results: The total sample size was 357 patients. Of these, 66 were no longer active patients in the practice. 42 patients had previously taken aspirin, but no longer were. 249 patients were included in the data analysis. Of 249, a PPI was co-prescribed in 138 patients $(55 \%)$.

Discussion: Our audit revealed that 55\% of patients were appropriately coprescribed a PPI. Recent research into the risks of PPIs may deter general practitioners from prescribing these medications. PPIs have been associated with increased risk of community acquired pneumonia, clostridium difficile associated diarrhoea and osteoporotic fractures in the elderly. These risks, as well as the fear of polypharmacy, may be a barrier to prescription. However as per current NICE guidelines, it is important to ensure that we are providing adequate gastro-protection to the elderly in order to avoid gastro-intestinal haemorrhage. Conclusion: More work needs to be carried out to ensure adherence to current guidelines. A re-audit will be carried out in October 2020 to ensure quality improvement in order to prevent adverse events in the elderly.

References:

1. Huang E, Strate L, Ho W, Lee S, Chan A. Long-Term Use of Aspirin and the Risk of Gastrointestinal Bleeding. The American Journal of
Medicine [Internet]. 2011;124(5):426-433. Available from: https://www. ncbi.nlm.nih.gov/pubmed/21531232

2. Huang W, Saver J, Wu Y, Lin C, Lee M, Ovbiagele B. Frequency of Intracranial Hemorrhage With Low-Dose Aspirin in Individuals Without Symptomatic Cardiovascular Disease. JAMA Neurology [Internet]. 2019;76(8):906. Available from: https://www.ncbi.nlm.nih.gov/pubmed/ 31081871

Considerations In Surgical Versus Nonsurgical Management Of Head And Neck Merkel Cell Carcinoma

Ni Fhlatharta $\mathrm{M}^{1}$, Crotty $\mathrm{T}^{1}$, McHugh $\mathrm{A}^{2}$, Moran $\mathrm{T}^{2}$.

Dept. of Thoracic Surgery ${ }^{I}$, Mater Misercordiae University Hospital, Dept. of Otorhinolaryngology ${ }^{2}$, St. Vincent's University Hospital, Dublin.

\section{$\mathrm{DML} / \mathrm{UCD}$}

Background: MCC is a rare cutaneous neuroendocrine tumour with a high risk of regional and distant metastasis. Management of this highly aggressive tumour is complex and mandates a multidisciplinary approach for optimal outcomes.

Method: Over 12 months, three cases of MCC in the cervicofacial region were identified and described. A critical review of the literature is included.

Results: Tumour staging confirmed $2 / 3$ cases had regional spread. One patient underwent unilateral parotidectomy with modified radical neck dissection and adjuvant radiotherapy. The other case is awaiting radiotherapy following a selective neck dissection. The final case had no further surgery and is currently under surveillance.

Conclusions: This case series illustrates the diversity in managing cervicofacial MCC's at various stages of their development.

\section{Tuberculosis Uveitis- Challenges in Diagnosis}

Connolly L, Neary S, Hurley C

Dept. of Ophthalmology, University Hospital Limerick

MW/UL

Introduction: According to the World Health Organisation TB remains one of the top ten causes of death worldwide. Interest in the disease has increased in recent years due to multidrug-resistant TB and international migration. Ocular TB occurs in $0.6-10 \%$ of cases and can be primarydirect invasion of the eye from the external surface or secondary- by either haematogenous spread of the bacteria or an immune reaction from infection causing inflammation.

Case Presentation: ZM, a 33-year old male from Bangladesh, was referred from his opticians to the ophthalmology clinic with a two-month history of progressive blurry vision in his right eye. He had no previous medical conditions but reported 'flu like' symptoms two months prior. Physical exam revealed anterior cervical lymphadenopathy. Ophthalmic examination revealed a non-granulomatous panvasculitis, with inflammatory activity in the anterior chamber, vitreous and retinal vasculitis. While these ophthalmic findings were not strongly indicative of TB, the ophthalmologist maintained a high index of suspicion considering his history and physical examination. ZM was referred for further investigations and a lymph node biopsy helped diagnose TB lymphangitis. He responded well to anti-TB medication and a reducing dose of steroids.

Discussion: This case highlights the importance of a comprehensive approach to patients presenting with uveitis, which considers the clinical history, physical, and ophthalmic examination in guiding further investigations While certain ophthalmic findings, such as granulomatous cells, are more suggestive of ocular TB, its manifestation in the eye can vary. Definitive diagnose involves a positive culture from ocular fluids, which is rarely practiced clinically. The diagnosis therefore is often presumptive, defined as ${ }^{(1)(2)}$ 
1. Intraocular findings suggestive of TB for which other causes have been excluded

2. Strongly positive tuberculin skin test, radiological evidence of TB or evidence of extrapulmonary involvement by tissue culture or microscopic examination

3. Response to anti-TB therapy

References:

1. Gupta V, Gupta A, and Rao N. Intraocular tuberculosis - an update. Surv Ophthalmol 2007; 52: 561-587.

2. Abu El-Asrar AM, Abouammoh M, Al-Mezaine HS. Tuberculous uveitis. Middle East Afr J Ophthalmol 2009;16:188-201

\section{Audit On The Prescription Of Bone Protection In The Elderly}

Jamil IJ, O'Mara G

Dept. of Medicine, Roscommon University Hospital.

\section{WNW/NUIG}

Background and Aims: Osteoporosis is a disease characterised by low bone mass and structural deterioration of bone tissue, with a consequent increase in bone fragility and susceptibility to fracture. The prevalence of osteoporosis increases significantly with age, from $2 \%$ at 50 years old to $25 \%$ at 80 years old. Fragility fractures are fractures that result from mechanical forces that would not ordinarily result in fracture, known as low-level trauma. NICE guidelines recommend that patients at risk of a fragility fracture be assessed and considered for bone protection. The aim of this audit is to assess whether or not the current practice in Roscommon University Hospital adheres to these guidelines.

Method: The audit was done on all inpatients on the date of $18^{\text {th }}$ November 2019. A kardex review was conducted for data collection and a chart review was also done to identify those with risk factors for osteoporosis. The data collected included age, gender, BMI and previous medical history. A statistical analysis was done and current practices were compared to the available guidelines from NICE and NOGG.

Results: A total of 31 patients were included in the audit, age range from 53-91 years old. All patients had at least one risk factor for osteoporosis. 16 patients $(51.6 \%)$ were prescribed a form of bone protection. There was no documented usage of the FRAX tool to assess for risk of fragility fractures.

Conclusion: The adherence to available guidelines is currently poor and can be improved upon. As FRAX can only be used up to the age of 90, an alternative screening and risk stratification tool may be developed for use in the hospital during admission for future inpatients.

\section{When Less Is Sometimes More: A Case Of Arterial Thoracic Outlet Syndrome}

\section{McDonnell B, Power Foley M, McDonnell C}

Vascular Surgery Department, Mater Misericordiae University Hospital, Dublin

\section{$\mathrm{DML} / \mathrm{UCD}$}

Background: Thoracic Outlet Syndrome (TOS) was formerly called Scalenus Anterior Syndrome and attributed to subclavian artery compression by the anterior scalene muscle (ASM). Today, TOS encompasses a constellation of upper limb symptoms caused by compression of varying neurovascular structures by musculoskeletal elements of the thoracic outlet. Arterial TOS accounts for $1 \%$ of cases (1), caused by an impinged subclavian artery (SA) becoming aneurysmal or stenotic. Definitive management by thoracic outlet decompression with first rib resection $+/$ - interposition graft repair of a SA aneurysm can be considered, however these procedures carry a significant risk of permanent neurovascular damage.

Case Details: A 50-year-old woman presented with an acutely ischaemic right upper limb, initially managed with a brachial artery embolectomy and therapeutic anticoagulation. Post-operatively, she had persistent paraesthesia and pallor in the ulnar artery distribution, concerning for arterial TOS. On examination, she had no palpable ulnar pulse. A MRAngiogram and MR-Venogram revealed moderate-to-severe stenosis of the right subclavian artery, a patent right subclavian vein and no cervical rib.

After discussing management options, she opted for surgical intervention. She underwent exploration of the thoracic outlet via a supraclavicular approach with the intent of performing a first rib resection. Dissection of the SA revealed that the overlying ASM was highly adherent and the sole source of compression. The SA was skeletalised and ASM divided, to notable improvement. As the SA passed with sufficient space between the first rib and clavicle, and was not palpably calcified, no further intervention was deemed necessary. The patient's symptoms resolved within hours of surgery. Her post-operative course was uncomplicated and she was discharged on lifelong aspirin.

Conclusion: This case exemplifies how judicious intra-operative decision-making guided by anatomical findings can avoid a more morbid procedure while still achieving symptomatic relief.

\section{Reference:}

Sanders RJ, Hammond SL, Rao NM. Diagnosis of thoracic outlet syndrome. J Vasc Surg. 2007;46(3):601.

\section{Abdominal Aortic Aneurysm With Coinciding Horseshoe Kidney - Planning A Strategic Surgical Approach}

Low R, Power Foley M, O’Donohoe MK

Dept. of Vascular Surgery, Mater Misericordiae University Hospital, Dublin

\section{$\mathrm{DML} / \mathrm{UCD}$}

Introduction: The co-existence of abdominal aortic aneurysm (AAA) and horseshoe kidney (HK) creates a unique challenge for vascular surgeons. The risk of permanent renal failure is significant, regardless of approach. The main issue is how to manage the main renal arteries and any accessory renal arteries (ARAs) originating off the aneurysm sac. If sufficient renal perfusion is lost, this may result in renal infarction $+/$ - failure. Reimplantation of multiple ARAs at open repair or revascularising them via a custom-made fenestrated device at endovascular repair may be technically challenging and not guaranteed to preserve renal function.

Patient Details: A 66-year-old woman presented with a $5.6 \mathrm{~cm}$ AAA. Comorbidities included gross obesity, (BMI 40), hypertension and Type II Diabetes Mellitus with diabetic nephropathy. Baseline creatinine and estimated Glomerular Filtration Rate (eGFR) were $120 \mu \mathrm{mol} / \mathrm{l}$ and $43 \mathrm{ml} /$ $\min / 1.73 \mathrm{~m}^{2}$, respectively. Preoperative CT-Aortogram noted a HK with two dominant arteries arising from the aorta in the normal position, and three smaller ARAs supplying the isthmus arising directly off the aneurysm at the bifurcation. The AAA was otherwise suitable for a standard EVAR.

Investigations \& Results: A selective renal angiogram was performed to determine the perfusion field of each artery and concluded the two dominant arteries supplied $>50 \%$ of the HK. A DMSA scan indicated the isthmus was non-functional, while the left and right moieties provided equal function. With input from nephrology and radiology, EVAR was the chosen approach. Factors considered included the patient's body habitus, comorbidities, and the consequences of infarcting the non-functional isthmus. The patient had a successful and uncomplicated procedure using a Bolton Graft. Post-operative CT-EVAR demonstrated no endoleak or renal ischaemia. By discharge, creatinine and eGFR were at baseline.

Conclusion: Multidisciplinary collaboration and thorough preoperative work-up aids surgical decision-making in difficult cases. 


\section{Fournier's Gangrene - One Woman's Journey}

Boyle E, Ridgway P

Dept. of General Surgery, Tallaght University Hospital

\section{DSE/TCD}

Introduction: Fourniers gangrene (FG) is a well known but rare disease occuring when necrotising fasciitis affects the genitals or perineum. It is a surgical emergency requiring urgent debridement. It has a mortality rate of $20-30 \%{ }^{1}$. It is approximately 10 times more common in men than in women ${ }^{2}$. Risk factors for developing FG include diabetes, alcoholism and treatment with SGLT2 inhibitors ${ }^{3,4}$.

Case Report: We present a case of a 58-year old woman admitted with a one week history of pain in the vulval/perineal region. She had a background history of non-insulin dependent type 2 diabetes but with poor blood glucose control. Her regular medications were dampagliflozin, atorvastatin, glicazide, metformin, sitagliptin and valsartan. On examination a large area of erythema involving the left groin, anterior thigh and left buttock was noted. Crepitus was also appreciated on examination. She had grossly raised inflammatory markers and a lactic acidosis on admission. The patient was started on intravenous antibiotics and her diabetic medication was held. She underwent surgical debridement which found necrotic fascia extending up to the lateral inguinal area and to the anterior vaginal wall. Extensive debridement was undertaken and she was admitted to the intensive care unit and remained intubated. Twelve hours later she returned to theater for a re-look procedure with extensive washout and further debridement. She was extubated following the re-look procedure and a vac dressing was fitted two days later in theatre. Postoperatively she improved, a urinary catheter was inserted and her antibiotics were rationalised as she recovered. She was discharged with an indwelling catheter and arrangements for twice weekly vac dressing changes until wound review in approximately three weeks.

Discussion: Early recognition and appreciation of the risk factors for Fournier's gangrene are crucial for the diagnosis and timely management of this potentially life threatening infection.

\section{References:}

Thwaini A, Khan A, Malik A, et al. Fournier's gangrene and its emergency management. Postgrad Med J. 2006;82(970):516-519. doi:10.1136/ pgmj.2005.042069

Eke N. Fournier's gangrene: a review of 1726 cases. Br J Surg 2002;87(6):718-28.

Chernyadyev S, A, Ufimtseva M, A, Vishnevskaya I, F, Bochkarev Y, M, Ushakov A, A, Beresneva T, A, Galimzyanov F, V, Khodakov V, V: Fournier's Gangrene: Literature Review and Clinical Cases. Urol Int 2018;:91-97. doi: 10.1159/000490108

Onder, C., Gursoy, K., Kuskonmaz, S., Kocer, U., \& Culha, C. (2019). Fournier's gangrene in a patient on dapagliflozin treatment for type 2 diabetes. Journal of Diabetes, 11(5), 348-350. https://doi.org/10.1111/ 1753-0407.12896

\section{Thymoma: A Case Report}

Ryan S, Power L, Gill C, Kennedy B

Acute Medical Assessment Unit,, St. James's Hospital

\section{DSE/TCD}

Introduction: Thymomas are rare neoplasms which arise in thymic epithelial tissue. They are the most common tumour of the anterior mediastinum in adults, however, are rare overall, with an annual incidence of 1.33.2 per million.

Case Report: A previously well 39-year-old woman presented following GP referral with an eight-week history of progressive shortness of breath and cough, with changes on chest $\mathrm{x}$-ray.
Her shortness of breath was initially on exertion, progressing to dyspnoea at rest. Her cough had initially been productive of green sputum, now dry. She had received several courses of antibiotics and steroids without much improvement in symptoms.

An outpatient chest X-ray had been ordered by the GP which showed a large opacification over the right mid and lower zones and advised CT thorax for further evaluation.

Following admission a CT thorax revealed a large, $10.8 \times 10.7 \times 10.2 \mathrm{~cm}$ rounded mass arising from the anterior mediastinum, abutting the SVC and right atrium, consistent with a thymoma. A CT guided biopsy confirmed the diagnosis of a thymoma, WHO type B1.

The patient was discharged and later admitted for an elective thymectomy under the Cardiothoracic surgeons.

Discussion: Thymomas present in a variety of different ways. One third present with paraneoplastic syndromes, most commonly Myasthenia gravis. One third with local symptoms such as cough and dyspnoea associated with the intra-thoracic mass. One third remain asymptomatic and are found incidentally.

Computed tomography is recommended to evaluate mediastinal masses and if the lesion is well circumscribed in keeping with a thymoma, a histological diagnosis is not necessary prior to surgery. A biopsy is deemed necessary in cases where the lesion is inoperable or involves surrounding structures, or where surgical resection may be incomplete.

Surgical resection is the gold standard of management. In inoperable cases, chemoradiotherapy can improve both symptoms and long-term survival.

Audit Into The Use Of Proton Pump Inhibitors And Their Association With Reduced Renal Function In A Medical Cohort Over A 1-Month Period

Neville J, Khan S

Galway Clinic, Galway

\section{WNW/NUIG}

Introduction: Proton pump inhibitors are commonly prescribed medications for the relief of dyspepsia and prevention of ulcer formation secondary to non-steroidal anti-inflammatory drugs (NSAIDs). Prolonged use of these medications carries the risk of reduced renal function secondary to interstitial nephritis.

Aims: To assess the proportion of patients taking PPIs, the age group in which PPIs are most commonly used, if PPIs were indicated and if the use of PPIs had any correlation to reduced renal function. In addition, the proportion of patients with chronic kidney disease (CKD) using PPIs on a regular basis was assessed.

Methods: A retrospective chart review was conducted on all medical admissions from January $1^{\text {st }}$ to January $31^{\text {st }} 2019$ inclusive. The age and sex of the patients, name of PPI, dose of PPI, renal profile and history of CKD of the patients taking PPIs was collected. As well as this, the indication for the PPI (gastritis, gastro-oesophageal reflux disease, peptic ulcer disease, gastrointestinal bleed and concurrent use of NSAIDs) and if the patient was taking the PPI prior to admission was recorded.

Results: Of 194 medical admissions, 84 patients were taking PPIs; 31 male and 53 female. 28 patients $(33.33 \%)$ had an indication to be taking a PPI. 67 patients $(79.76 \%)$ taking PPIs were aged 65 and over. 27 patients $(32.14 \%)$ who were taking PPIs had eGFR $<60.9$ patients $(10.71 \%)$ taking PPIs had a known history of CKD. All of those with CKD were aged 65 and over. 13 patients (15.47\%) were commenced on PPIs during their admission. The remainder were using PPIs prior to admission.

Conclusions: The results of this audit highlighted that PPIs are currently being over-prescribed, in particular in those over 65 and with renal impairment. An effort should be made to reduce the dose of PPIs in renal impairment or eliminate PPIs where they are not indicated. 
Waiting for Godot: How Long Are Older Patients Waiting in the Emergency Department Before Admission?

O'Riordan E, Coary R, Robinson D

Dept. of Medicine for the Elderly, St James' Hospital, Dublin

\section{DSE/TCD}

Background: Admission times from emergency departments (EDs) are an important indicator for clinical outcomes for patients. This is especially true for older patients, who often present with frailty associated conditions. The Irish Association for Emergency Medicine targets a 6 hour wait time for $95 \%$ of patients from presentation to admission or discharge (1). The aim of this audit was to review our own practice against this national standard for all patients admitted from ED under the care of the Medicine for the Elderly (MedEl) teams.

Methods: All patients referred to MedEl for the month of August 2019 were included (typically $\geq 65$ years old), but also included stroke patients (all ages). Data were collected from the local ED statistics office. The time points recorded were time of initial presentation and full admission.

Results: 114 patients were admitted under MedEL in August 2019; 21 were fully admitted within 6 hours $(18 \%)$. The mean time from initial presentation to admission to a ward was 9 hours 45 minutes. The longest time was 24 hours 42 minutes and the shortest time was 1 hour 5 minutes. The mean time in ED prior to referral to MedEl was 4 hours and 23 minutes. The mean time from referral to MedEl to ward admission was 5 hours and 10 minutes.

Conclusion: Currently, we are not meeting the standards for admission times. This is particularly relevant when managing older, frailer patients who are at higher risk of adverse outcomes. However, this is a widespread issue nationally as reflected in HSE Performance reports. Further investigation is required to identify areas for improvement and a re-audit should take place at a suitable interval.

Reference:

The Emergency Medicine Programme for Ireland 2012

Adherence To Clinical Guidelines And Practice Patterns Among Patients Undergoing Haemodialysis In The Irish Health System

Leneghan C, Browne LD, Stack AG

Dept. of Renal Medicine, University Hospital Limerick

\section{MW/UL}

Background: The Kidney Disease Improving Global Outcomes (KDIGO) initiative have published clinical practice guidelines with a view to improving patient outcomes. For patients undergoing haemodialysis (HD), greater adherence to clinical targets specified by KDIGO is associated with lower mortality (1). International data confirm that substantial variation exists across countries. To date there is a paucity of data on Irish compliance rates. This national audit sought to assess adherence rates to clinical guidelines among Irish patients receiving HD. Method: Data was obtained from the National Kidney Disease Clinical Patient Management System (KDCPMS). Adult ( $\geq 18$ years) HD patients who had received a minimum of six HD sessions per month in the fourth quarter of 2015 were included in the study. Anonymised data was extracted on patient demographics, HD vintage, blood pressure control, measures of anaemia, metabolic acidosis, mineral bone disease, and dialysis efficiency. Compliance with KDIGO guidelines was assessed and compared across all centres.

Results: A total of 1,258 prevalent HD patients were identified across 15 renal centres in Ireland. $57.2 \%$ of patients achieved target blood pressure with significant inter-centre variation ranging from $30.7-71.6 \% .59 .1 \%$ were within the guideline target for Haemoglobin. Regarding control of bone disease indicators, only $22.3 \%$ of patients had simultaneous attainment of the target ranges for calcium, serum phosphate and $\mathrm{PTH}$, and $7.3 \%$ had none of these parameters within the target ranges.
Conclusion: This first national audit found low adherence rates to several quality indicators among patients undergoing $\mathrm{HD}$ with significant centerto centre variation. These low rates are likely to increase mortality rates in the Irish HD population. The prognostic impact of these findings will now be explored in a national cohort study as part of a new quality improvement initiative.

Reference:

Tentori F, Hunt WC, Rohrscheib M et al. Which targets in clinical practice guidelines are associated with improved survival in a large dialysis organization? J Am Soc Nephrol 2007; 18: 2377-2384

\section{Case Report: Challenges In The Management Of Chronic Pulmonary Aspergillosis}

Hughes K, O’Brien S, McLaughlin A

Dept. of Respiratory Medicine, St James' Hospital, Dublin

\section{DSE/TCD}

Introduction: Chronic Pulmonary Aspergillosis (CPA) affects approximately 3 million people worldwide. The standard first line treatment is with azole antifungals.

Case Presentation: We report the case of a 43-year-old male who presented with an episode of life-threatening massive haemoptysis on a background of known Chronic Pulmonary Aspergillosis. The patient had a long standing left upper lobe aspergilloma and bronchiectasis secondary to childhood Pertussis. While receiving treatment with azole antifungals the patient experienced adrenal suppression, which may have been the result of steroid potentiation or as a direct complication of azoles. This necessitated the cessation of azole antifungal therapy.

The patient then presented with what was his second episode of massive hemoptysis. Patient was treated with caspofungin under guidance by microbiology however this was felt to be suboptimal. Interventional radiology could not attempt embolisation due to torturous vessels and risk of anterior spinal artery syndrome. The patient was reviewed by surgeons who subsequently performed a successful left sided pneumonectomy (the entire left lung had to be removed due to adhesions).

Discussion: This case highlights the challenges faced in the management of this condition. Adverse effects are common with azole antifungal medications and treatment options thereafter are often limited. This patient was then faced with the difficult decision of choosing between the risk of massive haemoptysis in the future and major cardiothoracic surgery. Newer treatment modalities, such as transbronchial removal of intracavitary aspergilloma, have been described in case series and represent exciting new options in the management of CPA which may allow some patients to avoid surgery.

\section{Hydatid Cyst: A Case Report}

Ryan S, Power L, Gill C, Kennedy B, Kent B

Acute Medical Assessment Unit,, St. James's Hospital

\section{DSE/TCD}

Introduction: Cystic echinococcosis is a zoonotic disease caused by Echinocossus granulosus. Transmission to humans occurrs through ingestion of faeces containing eggs. The liver is the commonest organ infected with the lungs the second most common.

Echinococcus granulosus is rare in Ireland. Presentations include anaphylaxis from a ruptured cyst, local symptoms e.g. pain, or asymptomatic incidental findings.

Case Report: A previously well 28-year-old woman, originally from Moldova, presented with an episode of collapse. She fulfilled SIRS criteria with presumed but no identifiable infectious cause. She was treated empirically for sepsis. 
On day five of admission she reported a recent hospital admission in Moldova due to parasitic infection and liver cyst. A liver ultrasound revealed a $10.6 \times 9.3 \mathrm{~cm}$ univesicular cyst in keeping with a hydatid cyst. Albendazole was commenced and a pigtail drain inserted. A fluid sample contained protoscoleces of Echinococcus granulosus.

The drain was removed after one week and she was discharged with ongoing albendazole treatment. She is being considered for PAIR (Puncture Aspiration Injection Re-aspiration).

Discussion: The treatment of echinococcosis can involve medical, surgical and percutaneous interventional management.

Treatment with benizimidazole agents alone are often inadequate for complete resolution. Medical therapy is therefore used for early stage cysts, inoperable cysts or in combination with surgery or percutaneous procedures.

Surgical management has long been considered the first-line, definitive management option for echinococcosis. Options include radical or conservative surgery with radical surgery having lower recurrence rates. However, PAIR has become increasingly used for percutaneously accessible and less complex cysts and PAIR with medical treatment has higher cure rates, lower recurrence, and shorter length of stay than surgery alone. Inactive cysts are often management by the watch and wait approach. Follow up is recommended with yearly ultrasound imaging. Recurrences have been reported despite treatment for up to ten years.

\section{A Rare Case Of Refractory Gastrointestinal Bleed Secondary To Jejunal Diverticulosis In A Kidney Transplant Patient}

Dhannoon A, Keady C, Hecht D, Joyce M

Dept. of Colorectal Surgery, Galway University Hospital

\section{WNW/NUIG}

Introduction: Jejunal diverticulosis is a rare entity comprising of multiple outpouchings of the mesenteric border of the bowel with a prevalence of $0.3-1.3 \%$ on autopsy studies. 1 They are pulsion diverticulae due to herniation at the site of blood vessel penetration through the bowel wall. They are usually asymptomatic but very rarely they would present with complications with intestinal obstruction being the most common followed by perforation and refractory bleeding. 1,2

Case Description: A 52-year old, kidney transplanted, male with an intractable lower gastrointestinal bleed was transferred to our centre from a peripheral hospital. He was haemodynamically unstable, receiving high dose inotropes and vasopressors. He already received a massive blood transfusion. In spite of this, he remained acutely unstable. Emergency ontable OGD and colonoscopy were performed, suggesting a jejunal or ileal source of his bleeding. He subsequently underwent an emergency laparotomy which revealed extensive blood-filled, globular jejunal invaginations located on the mesenteric border. These began approximately $30 \mathrm{~cm}$ distal to the duodenojejunal junction. This segment of bowel was isolated by tying off the proximal and distal ends. Several proximal diverticua aspirated and decompressed were observed to rapidly refill with blood. This indicated that the bleeding was located within the isolated bowel segment.

A $77 \mathrm{~cm}$ segment of bowel was resected using a stapling device proximally and distally. Primary anastomosis was not deemed safe at the time due to the high dose of inotropes the patient was receiving. Furthermore, his immunosuppression and coagulopathy status made the case more challenging to manage. The patient was returned to theatre 6 days later for a 'relook' laparotomy and an end-to-side stapled anastomosis was performed. The patient had an uneventful post-operative recovery thereafter.

Post-operative histopathological assessment of the resected specimen revealed multiple small intestinal diverticula comprising of sac-like invaginations of the mucosal and submucosal compartments. There was attenuation of the muscularis propria, in keeping with jejunal diverticulosis.
Discussion: Jejunal diverticulosis proves a diagnostic challenge. Cases of refractory gastrointestinal haemorrhage require prompt surgical approach. Moreover, treating transplant patient in emergency settings is associated with significant morbidity and mortality and should be approached with extreme caution.

\section{Reference:}

Fisher JK, Fortin D. Partial small bowel obstruction secondary to ileal diverticulitis. Radiology 1977;122:321-322 2) Longo WE1, Vernava AM $3^{\text {rd }}$. Clinical implications of jejunoileal diverticular disease. Dis Colon Rectum. 1992 Apr;35(4):381-8

\section{A Misdiagnosis Of Mumps}

Ward. C, Kenny. G, Feeney. E

Infectious Disease Dept., St. Vincent's University Hospital, Dublin

\section{$\mathrm{DML} / \mathrm{UCD}$}

Background: A seventeen-year old, previous healthy rugby player presented to a private Emergency Department with a two week history of groin pain, and leg stiffness. He had no sexual partners and was fully vaccinated. His brother had been diagnosed with mumps eight months previous. His CRP was elevated and he was febrile. Mumps Ig G was positive but Ig M was negative. He was diagnosed with mumps orchitis and discharged home on seven days of amoxicillin.

Case Description: Ten days later he presented to the Emergency Department of SVUH with a worsening limp and fevers of $38.6^{\circ} \mathrm{C}$. He was tender suprapubically and in the left iliac fossa. Testicular examination was normal. His CRP was elevated at $190 \mathrm{mg} / 1$ and an STI screen (including HIV and syphilis), blood and urine cultures were negative. Mumps serology was consistent with previous infection or vaccination. $\mathrm{CT}$ abdomen revealed thickening of the urinary bladder wall and surrounding infiltration of the perivesical fat. A MRI demonstrated septic arthritis of the symphysis pubis. Due to the location of the process and the absence of a drainable collection no sample was taken. He was commenced on empiric ceftriaxone $2 \mathrm{~g}$ IV daily and had a clinical and biochemical response with falling CRP. He was discharged on Outpatient Antibiotic Therapy to complete a six-week regimen. A repeat MRI performed at three months revealed complete resolution.

Conclusion: Pubic symphysis septic arthritis is rare. Although cultures were negative, Staphylococcus aureus is the main causative organism, presumably from haematogenous spread. It is most common in young athletes particularly football players owing to repetitive over adduction and twisting. (1) It is likely the previous antibiotics in this patient prevented the bacteria growing in blood cultures. Diagnosis requires a high index of suspicion as symptoms can mimic other conditions which occurred in this case.

\section{Reference:}

Mezouuar, Imane El, et al. "Septic Arthritis of the Pubic Symphysis: A Case Report." Pan African Medical Journal, vol. 18, 2014 https://www. ncbi.nlm.nih.gov/pmc/articles/PMC4236770/

\section{Rheumatoid Arthritis And The Lung - A Common Relationship With An Uncommon Outcome}

Ulaganathan H1 2, O'Sullivan C1, Redmond K3, Gaine S3, Mikulich O1 Letterkenny University Hospital, Kilmacrennan Rd, Ballyboe Glencar, Letterkenny, Co. Donegal; The Mater Misericordiae University Hospital, Dublin

\section{WNW/NUIG}

Rheumatoid arthritis is a debilitating disease with a wide spectrum of manifestations involving both intra and extra articular features. This case encapsulates the complexity of the condition and emphasizes the need for a multi-specialty approach. 
Mrs X presented in March 2019 with a 5-week history of a non-resolving lower respiratory tract infection despite repeated course of antibiotics in the community. Investigations revealed bilateral pneumonia, parainfluenza virus and left hydro-pneumothorax. CT thorax demonstrated thick-walled pleural cavity in left upper and lower lobes containing internal gas and fluid.

This was on a background of severe deforming seropositive rheumatoid arthritis since age 13 . The extent of this disease has included pulmonary nodule formation since 1997, recurrent pneuomothoraces, bronchiectasis and atlantoaxial instability.

The complex nature of the case warranted discussion with cardiothoracic team in the tertiary centre who recommended conservative management and surveillance. She responded well to antibiotic treatment initially. However, she had subsequent re-admissions with an nonresolving infection and hemoptysis.US guided aspiration of effusion confirmed it was exudative but there was no growth of organisms. The recurrence of symptoms and frequent readmissions marked a deduction that her background infection was merely suppressed rather than eradicated. A repeat CT Thorax showed similar findings to scan in April. Discussion at radiology-lung MDT confirmed a likely diagnosis of bronchopleural fistula secondary to cavitating rheumatoid nodule. This is an incredibly rare complication of rheumatoid arthritis. The patient was transferred to a tertiary centre for thoracotomy and omentoplasty. This case was further complicated by the cervical spine involvement of her condition, requiring orthopaedic input and spinal precautions perioperatively.

During the procedure, respiratory function declined and decision made to insert endo-bronchial valve with chest drain. Post operatively, pleural fluid grew aspergillus fumigatus, which was treated with voriconazole. Mrs X is due for a repeat omentoplasty in 2020.

\section{Phaeochromocytoma Crisis In Woman With Neurofibromatosis Type 1}

Youl G, Fennell D, Miller C, McQuaid S

Dept. of Endocrinology, Mater Misericordiae University Hospital, Eccles St, Dublin

$\mathrm{DML} / \mathrm{UCD}$

A 35-year old lady from Brazil presented to the emergency department with left-sided abdominal pain, palpitations, diaphoresis and anxiety. She had moved from Brazil to Ireland a few months previously with her 5-year old daughter and husband. She had been on the train that morning and had a sudden onset of sharp abdominal pain. On examination in the emergency department, she was tachycardic, around $140 \mathrm{bpm}$ and her systolic blood pressure was $150 \mathrm{mmHg}$. She had café au lait spots and neurofibromas on her chest and abdomen as well as axillary freckling. She spoke little English, however her husband was able to translate that she had been diagnosed with neurofibromatosis in childhood by dermatologists with no treatment and that recently, before moving to Ireland, she had had a previous similar episode where she went to the emergency department with palpitations and high blood pressure. She had been given the diagnosis of anxiety. She had gone to see a cardiologist as she was getting short of breath occasionally and was unable to run after her daughter or climb the stairs, but no further follow up was given. In ED she was sent for an emergency CT scan, after which, the patient became haemodynamically unstable. The patient had been given a beta-blocker and had become severely hypotensive. The CT showed a haemorrhagic lesion on the left adrenal gland, suggestive of a haemorrhagic phaeochromocytoma. The patient was rushed to ICU where she had two cardiac arrests requiring intermittent CPR, and eventual application of ECMO. The patient was diagnosed to be in a phaeochromocytoma crisis and was treated with alpha- and betablocking agents (phenoxybenzamine and phentolamine). She was kept under sedation until she was sufficiently alpha-blocked and stable; she was taken off the ECMO and optimized for adrenalectomy surgery.

\section{A Good Vein, Not A Great Vein: Pseudoaneurysm Of Femoral- Popliteal Bypass Vein Graft}

Hickey D, Power Foley M, Healy D, Gray C, O’Donohue MK Dept. of Vascular Surgery, Mater Misericordiae University Hospital, Dublin

\section{$\mathrm{DML} / \mathrm{UCD}$}

Introduction: Femoro-popliteal bypass with an autologous vein graft remains the gold-standard approach for symptomatic, longsegment superficial femoral artery occlusion. Varicosity is a relative contraindication in a vein conduit, due to risk of aneurysmal changes. However, in certain cases, it may still be preferable to prosthetic alternatives. Saphenous vein graft pseudoaneurysm is a well-documented phenomenon in coronary artery bypass grafting, however there is a paucity of reporting on this complication after peripheral arterial bypass surgery.

Case Details: A 76-year-old female claudicant underwent left femoralabove knee popliteal bypass with in-situ saphenous vein graft. Upon implantation, the vein was enlarged. Dilation was noted in the proximal portion of the graft on duplex one year post-operatively, and the patient continued to attend for annual surveillance. Twelve years later, the patient presented to the outpatients department with a large pulsating mass in the left anteromedial thigh. Though aneurysmal, the graft remained patent and pulsatile, and the patient had no ischaemic symptoms.

Investigations \& Management: Duplex US confirmed the mass to be a $5.6 \times 6.6 \mathrm{~cm}$ pseudoaneurysm with a partially thrombosed sac. The patient underwent open repair with excision of the aneurysmal segment and restoration of arterial continuity with end-to-end anastomosis. Good Doppler signals were obtained intra-operatively and graft was patent on post-operative duplex.

Conclusion: Pseudoaneurysm of vein graft is a rare but potentially fatal complication of lower limb bypass surgery. This case demonstrates that durable primary patency can be achieved with a varicose vein graft, and that the foreseeable complications may be remedied without compromising the graft. Furthermore, it highlights the role played by post-operative surveillance where early detection enables prompt intervention.

\section{Pituitary Mass Effect: A Case Of Malignant Masquerade}

Khalil R, Dablouk M, Javadpour M

Dept. of Neurosurgery, Beaumont University Hospital, Dublin

\section{$\mathrm{DML} / \mathrm{UCD}$}

Metastatic disease to the pituitary gland (PM) is a rare entity associated with a poor prognosis. The clinical presentation is variable, with many patients often asymptomatic which delays differentiation from other benign pituitary lesions. We present the case of an 87-year-old female with insidious deterioration in vision and hormonal dysfunction including hyperprolactinemia and syndrome of inappropriate anti-diuretic hormone secretion. Initial imaging revealed a suprasellar mass most consistent with a pituitary macroadenoma. The patient subsequently underwent imageguided endonasal endoscopic trans-sphenoidal debulking, where the final histopathological analysis revealed metastatic renal cell carcinoma consistent with an origin of renal primary. Of note, the patient had a history of left T2aN0M0 renal cell carcinoma twenty-four years prior, for which she underwent a radical nephrectomy and no adjuvant therapy. Postoperatively she had a noticeable improvement in her ocular symptoms. Her management involves a multidisciplinary team of medical oncology to screen for additional metastases, radio- oncology to determine if she is a candidate for adjuvant radiation therapy and endocrinology to correct post-operative hormonal abnormalities. PM represents only $1 \%$ of 
pituitary lesions, and $0.14-28.1 \%$ of all brain metastases. The two most common primary sites are breast and lung. Case reports suggest that only $2.6 \%$ of pituitary metastases are from a renal primary. PM has a poor prognosis, with a mean survival of 6.5 months after diagnosis. Surgical resection, adjuvant radiotherapy and hormone replacement significantly improve the quality of life but do not affect survival rates. Clinicians should consider the possibility of pituitary metastases in a patient with a previous history of malignancy presenting with pituitary mass effect and hyperpituitarism. This case highlights the importance of long-term follow-up in patients whose cancer has a predilection for haematogenous spread, and the role for multidisciplinary approach in its management.

Womb To Improve: Rapidly Progressive Glomerulonephritis In A Postmenopausal Woman With Pyometra

Hickey $\mathrm{D}^{1}$, Holland $\mathrm{J}^{2}$, Brennan $\mathrm{D}^{1}$, Pontré $\mathrm{J}^{1}$, O’Meara $\mathrm{Y}^{2}$

Depts. Of Gynaecology ${ }^{1}$, Nephrology ${ }^{2}$, Mater Misericordiae University Hospital, Dublin

\section{DML/UCD}

Background: Rapidly progressive glomerulonephritis (RPGN) is a clinical syndrome manifesting with features of glomerular disease on urinalysis and progressive loss of renal function. It is characterised histologically by extensive crescent formation. Three types based on features of immune deposits on immunofluorescence and the presence of associated antibodies exist.

Type 3 RPGN is an ANCA-associated vasculitis (AAV) characterised by the absence of immune deposits. AAV has an annual incidence of fifteen per million, with a median age of onset of 62 years ${ }^{1}$.

Case Report: A 68-year-old female presented with four-month history of vaginal discharge and constitutional symptoms. Her history was notable only for sacrospinous fixation for prolapse five months prior.

On examination, she was hypertensive with abdominal tenderness and purulent cervical discharge. Ultrasound showed pyometra and echogenic kidneys.

Her serum creatinine was elevated corresponding with an AKIN stage 3 acute kidney injury (AKI). A normocytic anaemia was also present.

The AKI was believed to be secondary to sepsis and was treated with aggressive intravenous fluids and antibiotics. The patient developed periorbital and pedal oedema, bibasal crepitations, systolic murmur, and worsening hypertension.

Urinalysis revealed haematuria and nephrotic range proteinuria with red cell casts on microscopy. MPO-ANCA titre was $>134 \mathrm{IU} / \mathrm{mL}$ indicative of highly active renal vasculitis.

Drainage of the pyometra allowed for swift empiric immunosuppression and renal biopsy, which showed pauci-immune crescentic disease. The patient was promptly treated with corticosteroids and cyclophosphamide. Discussion: Early diagnosis of AAV and initiation of appropriate therapy is essential to minimize irreversible renal injury. AAV is often nonspecific in presentation and progresses to end-stage renal disease (ESRD) is predicted by the patient older age, degree of anaemia and peak serum creatinine within one month ${ }^{2,3}$. Currently cyclophosphamide and glucocorticoids compromise the backbone of treatment; but emerging therapies with less undesireable side effects show promise.

References:

Vasculitis Ireland Network. (2019). Rationale for VINE, the vasculitis Ireland Network. [online] Available at: https://www.tcd.ie/medicine/ thkc/vasculitis/ [Accessed 9 Dec. 2019].

Flossmann O, Berden A, de Groot K, Hagen C, Harper I, Heijl C, et al. Long-term patient survival in ANCA-associated vasculitis. Ann Rheum Dis 2011; 70: 488 - 94

Sven Weidner, Steffen Geuss, Syrus Hafezi-Rachti, Andrea Wonka, Harald D. Rupprecht, ANCA-associated vasculitis with renal involvement: an outcome analysis, Nephrology Dialysis Transplantation, Volume 19, Issue 6, June 2004, 1403-1411

Booth AD, Almond MK, Burns A, Ellis P, Gaskin G, Neild GH, Plaisance M, Pusey CD, Jayne DR Pan-Thames Renal Research Group: Outcome of ANCA-associated renal vasculitis: A 5-year retrospective study. Am J Kidney Dis 41: 776-784, 2003

Uterine Rupture At 22+4 Weeks Of Gestation During IOL For TOP
After Previous Cesarean Section-A Case Report

Nazir Nazir S, Mahedvi T, Giumaa N

Dept. of Obstetrics and Gynaecology, Portiuncula University Hospital.

\section{WNW/NUIG}

Introduction: Uterine rupture during pregnancy is an extremely serious complication related to high morbidity and mortality indices both fetal and maternal ${ }^{1}$.

Case presentation- A 43-year-old female G5 P3+1, with past obstetric history of EmLSCS, ventouse, ectopic pregnancy and SVD, underwent NIPT at $10+3 / 40$ due to her age with low probability results. Her antenatal anomaly scan at $21+2 / 40$ showed strawberry skull with marked shortening of the long bones and a bowed femur and humerus. She was referred to two fetal medicine units in which skeletal dysplasia was confirmed, a diagnosis of Thanatophoric dysplasia was suggested and a TC ratio of 0.69 was estimated, findings congruous with being lethal. The following day, consented feticide intracardiac injection of $\mathrm{KCl}$ with subsequent asystole confirmation was done. Mifepristone $200 \mathrm{mg}$ was given for the following day and a plan for induction with Misporstol was made. Three days later, at $22+4 / 40$ she was admitted for induction. Overnight she developed intermittent severe pain and contractions with a constant background discomfort and had an APH of $420 \mathrm{ml}$ with low BP, for which she received Hartmann's and Gelofusine solution. The following morning, she became pale and a TAS showed no signs of intraabdominal fluid or scar rupture. Hours later a repeat scan for ongoing LUQ, LIQ pain, showed a $12 \mathrm{~cm} \times 12 \mathrm{~cm} \times 7 \mathrm{~cm}$ clot within the uterine cavity and placental separation. Haemorrhage protocol was followed, and she was transferred to OT for an emergency hysterotomy delivery/ potential abdominal hysterectomy. After incision, a uterine rupture and intact membranes were observed with an in-situ placenta. Following the delivery, repair of the rupture site was done, the patient was transferred to ICU and subsequently to the ward where she recovered.

Discussion- UR diagnosis is difficult due to its frequently nonspecific presentation. This case highlights some of its risk factors and the importance of early diagnosis and management.

Reference:

1.Gardeil F, Daly S, Turner MJ. Uterine rupture in pregnancy reviewed. Eur J Obstet Gynecol Reprod Biol. 1994 Aug. 56(2):107-10

\section{A Curious Case Of Late-Onset Pompe Disease}

Slattery F, Walsh R

Dept. of Neurology, Tallaght University Hospital, Dublin

\section{DSE/TCD}

Introduction: Pompe Disease is a rare, inherited, autosomal recessive, glycogen storage disorder due to mutations in the GAA gene encoding the acid alpha glucosidase enzyme on chromosome 17. This results in glycogen accumulation in lysosomes and cytoplasm causing tissue destruction. The classical infantile form usually presents with hypertrophic cardiomyopathy and hypotonia. The late-onset form typically presents with proximal myopathy, affecting respiratory function, but is marked in that there is no cardiac involvement. Clinically, late-onset disease is typified by a skeletal myopathy, eventually leading to respiratory failure. 
Enzyme Replacement Therapy is the treatment of choice for all forms of the disease with alglucosidase alfa.

Case Presentation: We present the case of a gentleman who first presented to Neurology Clinic at the age of 54 with a myopathic 'waddling' gait and a 10 year history of unexplained back pain. MRI imaging demonstrated some atrophy and fatty replacement of paraspinal muscles. He attested that his sister was also similarly affected. At this time, he was diagnosed with a likely autosomal recessive limb-girdle muscular dystrophy. Two muscle biopsies were performed and both were unremarkable staining for glycogen and dystrophinopathies were negative. Further studies were carried out at the Muscle Unit in Newcastle, which provided no diagnosis. He experienced further progression, with a deterioration in respiratory function and his swallow. He was subsequently recruited to the Whole Exome Sequencing project through UCD, which revealed no abnormalities.

He subsequently represented to the clinic with a marked left ptosis and difficulty with left gaze. This is consistent with Chronic Progressive External Ophthalmoplegia, which raised the possibility of a mitochondrial cytopathy - explaining the lack of genetic diagnosis in blood. A myopathy and mitochondrial panel was sent, which showed characteristic pathogenic mutations in the GAA gene, confirming the diagnosis of lateonset Pompe Disease. However, his alpha glucosidase levels were normal, in keeping with the low enzymatic activity and this gentleman's mild phenotype.

\section{Hypothyroidism In The Context Of Immune Checkpoint Inhibitors}

Scanlon E, Crowley R

Dept. of Endocrinology, St. Vincent's University Hospital, Dublin

\section{$\mathrm{DML} / \mathrm{UCD}$}

$\mathrm{Mr} \mathrm{X}$, is a 61 year old male who presented for a non-urgent endocrinology out-patient appointment for new hypothyroidism, recently diagnosed by his GP. This was on a background of metastatic melanoma. His metastatic melanoma is in remission with on-going treatment with Nivolumab. In clinic, TSH was 18.09 , T4 was 14.4 , T3 was 4.3 . He reported symptoms of fatigue and weight gain, but was otherwise well. He had no personal or family history of endocrinopathy and had completed his most recent cycle of Nivolimab two weeks previously. His GP started him on Eltroxin and referred him to endocrinology. Importantly, his ACTH and cortisol were not checked prior to beginning thyroid replacement therapy.

Discussion: Clinically significant endocrinopathies occur in approximately $10 \%$ of patients treated with systemic checkpoint inhibitors (1). Nivolomab and Ipilimab are the two most commonly used agents. CTLA4 blockade (ipilimumab) is associated with an increased incidence of hypophysitis (up to 10\%) and primary thyroid dysfunction. Anti-PD1/PDL1 agents (nivolumab and pembrolizumab) are predominantly associated with primary thyroid dysfunction from thyroiditis(1).

These endocrinopathies are most often not reversible and are managed by hormone replacement(2). One 154 patient retrospective study of metastatic melanoma patients treated with ipilimumab were evaluated and hypopituitarism persisted in $76 \%$ of these individuals(3). When replacing thyroid hormone it is important to first check ACTH levels and replace hydrocortisone prior to thyroid replacement therapy. This was not done in the case of $\mathrm{Mr} \mathrm{X}$, and is important to prevent adrenal crisis which can be fatal(4). The use of these drugs is likely to become more prevalent with their increased use across a wide variety of cancers and increased awareness is needed across all healthcare professionals.

\section{References:}

1.Scott ES, Long GV, Guminski A, Clifton-Bligh RJ, Menzies AM, Tsang VH. The spectrum, incidence, kinetics and management of endocrinopathies with immune checkpoint inhibitors for metastatic melanoma. Eur J Endocrinol. 2018;178(2):173-80.
2.Girotra M, Hansen A, Farooki A, Byun DJ, Min L, Creelan BC, et al. The Current Understanding of the Endocrine Effects From Immune Checkpoint Inhibitors and Recommendations for Management. JNCI Cancer Spectr. 2018;2(3):pky021-pky.

3.Faje AT, Sullivan R, Lawrence D, Tritos NA, Fadden R, Klibanski A, et al. Ipilimumab-Induced Hypophysitis: A Detailed Longitudinal Analysis in a Large Cohort of Patients With Metastatic Melanoma. The Journal of Clinical Endocrinology \& Metabolism. 2014;99(11):4078-85. 4.Murray JS, Jayarajasingh R, Perros P. Lesson of the week: Deterioration of symptoms after start of thyroid hormone replacement. BMJ (Clinical research ed). 2001;323(7308):332-3.

\section{A Case Of Achalasia In A Post Sleeve Gastrectomy Patient.}

Ó Coisdealbha P, Collins C

Dept. of Gastrointestinal Surgery, University Hospital Galway

\section{WNW/NUIG}

This report discusses a 43-year old female, two years post laparoscopic sleeve gastrectomy, referred to the Upper GI service UHG with progressive three month history of dysphagia to solid foods and new onset regurgitation for the past six weeks. On review she had an estimated Eckardt score of 6 . Since her surgery the patient had lost a total of $35 \mathrm{~kg}$ and had achieved a BMI of 33.8 from an original high of 48.6 .

This differential diagnosis of this case consisted of oesophageal dysmotility, benign oesophageal stricture and oesophageal carcinoma. The patient underwent gastroscopy, reported as being normal with gastritis noticed. High dose proton pump inhibitor was commenced with no improvement. Barium swallow, $\mathrm{pH}$ and manometry were performed to further evaluate the patients symptoms. These investigations identified elevated upper and lower oesophageal sphincter pressures inkeeping with achalasia. Treatment options discussed with the patient included botulinum toxin injection, pneumatic dilatation and surgical myotomy. Surgery had the significant issue of there being no fundus remaining for the creation of an anti-reflux fundlopification. Botulinum toxin injection in this case would require recurrent, repeated treatment every 3-6 months going forward. It was therefore agreed upon with the patient to undergo gastroscopy and balloon dilatation.

The patient underwent therapeutic treatment with Endoflip ${ }^{\mathrm{TM}}$ gastroscopy and balloon dilatation. The patients pre-dilatation diameters showed a narrowing of the oesophagus estimated at being $12.2 \mathrm{~mm}$ and a postdilatation diameter of $18.0 \mathrm{~mm}$. Following treatment the patient noticed an immediate surgical improvement but will need to be reassessed going forward.

Achalasia post laparoscopic sleeve gastrectomy has not been widely reported on in the literature. This case provided an opportunity to report this rare event not seen with laparoscopic sleeve gastrectomy and to discuss the variety of clinical and radiological investigations needed to achieve a diagnosis and to formulate a treatment plan.

\section{Documentation Of Patients' Social Circumstances In Medical} Patients' Admission Notes: A Clinical Audit

Amaechi G*, Tan J*, Akinpeloye H, Mulpeter K

Joint first authors*

Dept. of Medicine for the Elderly, Letterkenny University Hospital

\section{WNW/NUIG}

Background: As per "HSE standards and recommendations practices for healthcare records management", patients' social circumstances should be included in the admission notes ${ }^{1}$. Patients' social history is an important aspect in patient care and also in discharge planning. The aim of this clinical audit is to assess whether patient's domestic, employment and 
lifestyle circumstances are documented in the clinical admission notes and who it was documented by.

Methods: A review of 38 medical patients' admission notes was performed over a period of 4 weeks. The admission notes were either documented on the standardised hospital medical Admission Proforma or on a continuation sheet in the chart.

Among the inclusion criteria were:

Medical patients who were admitted within one week

Physical activity as a mandatory requirement in lifestyle documentation

Results: Out of 38 medical patients' admission notes, $3 \%$ was completed by interns, $63 \%$ by the senior house officers and $34 \%$ by the registrars.

Patients' domestic circumstances were documented adequately $79 \%$ of the time.

Patients' employment history was documented inadequately $55 \%$ of the time. No lifestyle circumstances were documented adequately in the admission notes. Conclusion: Most admission notes are completed by senior house officers. Social circumstances were generally included in the admission notes of patients, however not all aspects of the HSE standards were adhered to. The documentation of patients' social history is limited by the format of the medical Admission Proforma.

We recommend:

Expanding the space in the Proforma to record patient's employment status more comprehensively including retirement.

Including level of physical activity to the lifestyle history in the Proforma References:

Health Service Executive (2011). HSE Standards \& Recommended Practices for Healthcare Records Management. HSE, p.90.

\section{A Case Report Of Small Bowel Adenocarcinoma In An Ilial J-Pouch}

Conroy F, Doherty G

Centre for Colorectal Disease, St. Vincent's University Hospital, Dublin

\section{$\mathrm{DML} / \mathrm{UCD}$}

Case Presentation: A 74 year-old Caucasian male presented to OPD with a longstanding history of multiple loose bowel motions occurring both diurnally and nocturnally, with recent reduction of continence requiring constant pad wearing, on a background of longstanding J-pouch inflammation (formed secondary to Ulcerative Colitis) and coeliac disease.

His medical history consisted of panproctocolectomy with IPAA (Jpouch) 30 years ago for UC with multiple colorectal polyps, PSC, coeliac disease (non-compliant to dietary management), asthma, atrial fibrillation, adrenal insufficiency, previous perforated duodenal ulcer and a PSH of benign ampullary lesion removal and previous cholecystectomy. Of note he was prescribed immodium $2 \mathrm{mg}$ and is a non-smoker with a family history of CRC. Bloods showed a normal $\mathrm{Hb}$ and ferritin, with deranged electrolytes secondary to loose bowel motions, deranged LFT's secondary to PSC and elevated Anti-tTG antibodies.

Case Management: A pouchoscopy was performed which showed a diffusely inflamed pouch with a polypoid area of ulceration close to the inlet (video and images available). A biopsy showed predominantly low grade dysplasia but foci suspicious for high grade dysplasia (p53 overexpression). Given the long-standing pouchitis and dysplasia, pouch excision was performed with end ileostomy formation. Subsequent histology showed a $2 \mathrm{~cm}$ moderately differentiated small bowel adenocarcinoma (SBA) staged as T3N0M0 with complete margin resection.

Discussion: SBA is a rare cause of GI malignancy whose incidence is increasing and annually accounts for between $1-3 \%$ of all GI cancers ${ }^{\mathbf{1 , 2}}$, despite accounting for $90 \%$ of the mucosal surface ${ }^{3}$. It presents a diagnostic and therapeutic challenge and thus often results in diagnosis at an advanced stage ${ }^{2}$, with 5 -year overall survival $14-33 \%^{3}$. Key risk factors include Coeliac Disease and Crohns, as well as genetic and environmental factors $\mathbf{1 , 2}$. Moreover, dysplasia/malignancy is a recognised complication of pouch formation, with persistent inflammation an important risk factor in $\mathrm{UC}^{4}$.
Conclusion: This is a rare case of SBA which highlights the need for high clinical suspicion in patients with risk factors given the poor prognostic outcomes.

\section{References:}

Raghav, K., \& Overman, M. (2013). Small bowel adenocarcinomasexisting evidence and evolving paradigms. Nature Reviews Clinical Oncology, 10(9), 534-544. doi: 10.1038/nrclinonc.2013.132

Lech, G., Korcz, W., Kowalczyk, E., Słotwiński, R., \& Słodkowski, M. (2017). Primary small bowel adenocarcinoma: current view on clinical features, risk and prognostic factors, treatment and outcome. Scandinavian Journal Of Gastroenterology, 52(11), 1194-1202. doi: 10.1080/00365521.2017.1356932

Aparicio, T., Zaanan, A., Svrcek, M., Laurent-Puig, P., Carrere, N., \& Manfredi, S. et al. (2014). Small bowel adenocarcinoma: Epidemiology, risk factors, diagnosis and treatment. Digestive And Liver Disease, 46(2), 97-104. doi: 10.1016/j.dld.2013.04.013

Ng, K., Gonsalves, S., \& Sagar, P. (2019). Ileal-anal pouches: A review of its history, indications, and complications. World Journal of Gastroenterology, 25(31), 4320-4342. doi: 10.3748/wjg.v25.i31.4320

\section{Utilising 3d Ct-Reconstruction For Multidiscilpinary Planning In Complex Hip Revision Surgery}

Koay WJ ${ }^{1}$, Vukanic $\mathrm{D}^{1}$, Tahir $\mathrm{A}^{1}$, McNulty $\mathrm{G}^{2}$, Murphy $\mathrm{C}^{1}$ Depts. of Orthopaedics ${ }^{1}$, Radiology ${ }^{2}$, University Hospital Galway

\section{WNW/NUIG}

Introduction: With increasing numbers of total hip arthroplasties (THA) performed, as well as in younger cohorts, the number of revision-THAs is also rising. Revisions involving the acetabular component ( $>50 \%)$ are implicated due to osteolysis, loosening, dislocation, migration, pain, infection or malposition. Current imaging trends involve plain radiographs as the first line imaging of choice, and Computed Tomography (CT) scans which are used for classification of acetabular bone loss and surgical planning. We present a case series utilizing 3D reconstructions of available CT scans to improve the accuracy of classifying acetabular fractures/ bone loss, increase diagnostic confidence, and for multidisciplinary surgical planning.

Case Series: We report 4 cases of acetabular component defects caused by loosening, infection, migration and dislocation, comparing the modalities of imaging (plain radiograph, $\mathrm{CT}$, and 3D-reconstruction) performed and highlight the value of the 3D-reconstructive images in classifying acetabular bone loss and surgical planning for specific case scenarios.

Discussion: The 3D reconstruction shown in the case series can be performed as part of the investigative work-up of the patients at no extra cost. It enables the ability for 360-degree rotation of the 3D images on multiple planes, and also allows for better appreciation of the anatomical relationship between the metalwork, bony structures and vasculature of the pelvis, previously unobtainable via conventional radiology.

Conclusion: We believe that $3 \mathrm{D}$ reconstruction of $\mathrm{CT}$ imaging can play a significant role in accurately characterizing periprosthetic fractures involving the acetabulum, as well as in pre-operative surgical planning for revision surgery. There is also an educational role where 3D reconstruction acts as an important adjunct in helping NCHDs visualize the anatomical injuries to the surgical team, with the help of pre-existing classification.

\section{A SEA-rious Reaction}

McCarthy R, Ali G

Dept. of General Medicine, Bantry General Hospital

\section{$\mathrm{S} / \mathrm{UCC}$}

Introduction: An allergy is defined as a chronic condition whereby the immune system reacts abnormally to an ordinarily harmless substance called an allergen. This reaction can manifest as urticaria, angioedema 
or anaphylaxis. This case describes the presentation of a lady with angioedema and diffuse urticaria following open water swimming.

Case: A 54-year old lady presented to Bantry General Hospital Medical Assessment Unit on a GP referral of "Angioedema and diffuse urticaria on her legs and trunk". She reported swimming for 10 minutes in the sea when her lips began to swell and a pruritic rash appeared on her legs and trunk. She had an atopic background of asthma for which she was taking Symbicort and Salbutamol inhalers. She had no other personal or family history of atopy. Her family history was significant for a brother with Multiple Sclerosis and a daughter with Ankylosing Spondylitis. She reported a recent sensitivity to cold which began two weeks prior whereby she experienced stinging, painful skin when exposed to cold air.

Her GP administered IM Piriton with a good response prior to her MAU presentation. On examination, her vitals were within normal limits. She had slight prominence of her lower lip and neckline erythema with no signs of respiratory distress. Routine laboratory bloods were normal. A Cold Stimulation Test was positive with a geographical wheal appearing on her left forearm in the distribution of an ice pack that had been placed on it. She was discharged on regular Cetirizine 10mg OD and an EpiPen $300 \mathrm{mcg}$. She was advised against cold water swimming and was subsequently referred to an immunologist.
Discussion: Cold urticaria can be either acquired or familial. Acquired cold urticaria has an incidence of $0.05 \%$ and is more common than familial. Symptoms include urticaria, angioedema and occasionally anaphylaxis. Open water swimming is the most common trigger and it is cold sensitivity test positive. The diagnosis is primarily clinical and it is treated with antihistamines, systemic steroids and epinephrine.

Familial Cold Autoinflammatory Syndrome is autosomal dominant. Symptoms include urticaria, fever and joint pain. It does not respond to the cold stimulation test. There is an autoimmune component which is interesting given the family history in this case. Diagnosis involves testing inflammatory marker levels, a skin biopsy or genetic testing. It is treated with biologic agents.

This case demonstrates the relationship between autoimmunity and allergy.

Publisher's note Springer Nature remains neutral with regard to jurisdictional claims in published maps and institutional affiliations. 